\title{
Complexity Analysis of Generalized and Fractional Hypertree Decompositions
}

\author{
GEORG GOTTLOB and MATTHIAS LANZINGER, University of Oxford, United Kingdom and
}

TU Wien, Austria

REINHARD PICHLER, TU Wien, Austria

IGOR RAZGON, Birkbeck University of London, United Kingdom

\begin{abstract}
Hypertree decompositions (HDs), as well as the more powerful generalized hypertree decompositions (GHDs), and the yet more general fractional hypertree decompositions (FHDs) are hypergraph decomposition methods successfully used for answering conjunctive queries and for solving constraint satisfaction problems. Every hypergraph $H$ has a width relative to each of these methods: its hypertree width $h w(H)$, its generalized hypertree width $g h w(H)$, and its fractional hypertree width $f h w(H)$, respectively. It is known that $h w(H) \leq k$ can be checked in polynomial time for fixed $k$, while checking $g h w(H) \leq k$ is NP-complete for $k \geq 3$. The complexity of checking $f h w(H) \leq k$ for a fixed $k$ has been open for over a decade.

We settle this open problem by showing that checking $f h w(H) \leq k$ is NP-complete, even for $k=2$. The same construction allows us to prove also the NP-completeness of checking $g h w(H) \leq k$ for $k=2$. After that, we identify meaningful restrictions that make checking for bounded $g h w$ or $f h w$ tractable or allow for an efficient approximation of the $f h w$.
\end{abstract}

CCS Concepts: $\bullet$ Information systems $\rightarrow$ Relational database query languages; $\bullet$ Theory of computation $\rightarrow$ Problems, reductions and completeness;

Additional Key Words and Phrases: Generalized hypertree decompositions, fractional hypertree decompositions, np-hardness, tractable fragments, multi-intersection width, hypergraphs, candidate tree decompositions

\section{ACM Reference format:}

Georg Gottlob, Matthias Lanzinger, Reinhard Pichler, and Igor Razgon. 2021. Complexity Analysis of Generalized and Fractional Hypertree Decompositions. J. ACM 68, 5, Article 38 (September 2021), 50 pages.

https://doi.org/10.1145/3457374

This is a significantly extended and enhanced version of a paper presented at PODS 2018 [24].

The authors acknowledge support by the Austrian Science Fund (FWF): Projects P30930 and Y698. Georg Gottlob is a Royal Society Research Professor and acknowledges support by the Royal Society in this role through the "RAISON DATA" project (Reference No. RP\R1\201074). Matthias Lanzinger acknowledges support by the Royal Society "RAISON DATA" project (Reference No. RP $\backslash$ R1 $\backslash 201074)$.

Authors' addresses: G. Gottlob and M. Lanzinger, University of Oxford, Department of Computer Science, Oxford, UK, TU Wien, Vienna, Austria; emails: \{georg.gottlob, matthias.lanzinger\}@cs.ox.ac.uk; R. Pichler, TU Wien, Vienna, Austria; email: reinhard.pichler@tuwien.ac.at; I. Razgon, Birkbeck University of London, London, UK; email: igor@dcs.bbk.ac.uk. \footnotetext{
4.0 License.

(c) 2021 Copyright held by the owner/author(s).

0004-5411/2021/09-ART38 \$15.00

https://doi.org/10.1145/3457374
}

This work is licensed under a Creative Commons Attribution-NonCommercial-ShareAlike International 


\section{INTRODUCTION AND BACKGROUND}

Research Challenges Tackled. In this work, we tackle computational problems on hypergraph decompositions, which play a prominent role for answering Conjunctive Queries (CQs) and solving Constraint Satisfaction Problems (CSPs), which we discuss below.

Many NP-hard graph-based problems become tractable for instances whose corresponding graphs have bounded treewidth. There are, however, many problems for which the structure of an instance is better described by a hypergraph than by a graph, for example, the above mentioned CQs and CSPs. Treewidth does not generalize hypergraph acyclicity (sometimes also referred to as $\alpha$-acyclicity, see e.g., References [22, 54]). Hence, proper hypergraph decomposition methods have been developed, in particular, hypertree decompositions (HDs) [31], the more general generalized hypertree decompositions (GHDs) [31], and the yet more general fractional hypertree decompositions (FHDs) [35], and corresponding notions of width of a hypergraph $H$ have been defined: the hypertree width $h w(H)$, the generalized hypertree width $g h w(H)$, and the fractional hypertree width $f h w(H)$, where for every hypergraph $H, f h w(H) \leq g h w(H) \leq h w(H)$ holds. Definitions are given in Section 2. A number of highly relevant hypergraph-based problems such as CQ-evaluation and CSP-solving become tractable for classes of instances of bounded $h w$, ghw, or, $f h w$. For each of the mentioned types of decompositions it would thus be useful to be able to recognize for each constant $k$ whether a given hypergraph $H$ has corresponding width at most $k$, and if so, to compute such a decomposition. More formally, for decomposition $\in\{\mathrm{HD}, \mathrm{GHD}, \mathrm{FHD}\}$ and $k>0$, we consider the following family of problems:

СНеск (decomposition, $k$ )

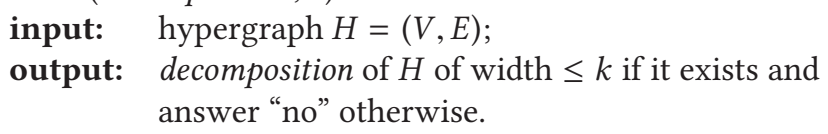

As shown in Reference [31], Снеск(HD, $k$ ) is in Ptime for every fixed $k$. However, little is known about $\mathrm{CHECK}(\mathrm{FHD}, k)$. In fact, this has been an open problem since the 2006 paper [34], where Grohe and Marx state: "It remains an important open question whether there is a polynomialtime algorithm that determines (or approximates) the fractional hypertree width and constructs a corresponding decomposition." Since then, the approximation problem has been resolved [44] Regarding the problem of determining the exact width, the 2014 journal version [35] still mentions this as open and it is conjectured that the problem might be NP-hard. The open problem is restated in Reference [51], where further evidence for the hardness of the problem is given by showing that "it is not expressible in monadic second-order logic whether a hypergraph has bounded (fractional, generalized) hypertree width.” We will tackle this open problem here:

Research Challenge 1: Is СНEск(FHD, $k$ ) tractable?

Let us now turn to generalized hypertree decompositions. In Reference [31] the complexity of $\operatorname{CHEсK}(\mathrm{GHD}, k)$ was stated as an open problem. In Reference [32], it was shown that Снеск (GHD, $k$ ) is NP-complete for $k \geq 3$. For $k=1$ the problem is trivially tractable because $\operatorname{ghw}(H)=1$ just means $H$ is acyclic. However, the case $k=2$ has been left open. This case is quite interesting, because it was observed that the majority of practical queries from various benchmarks that are not acyclic have $g h w=2[11,23]$, and that a decomposition in such cases can be very helpful. Our second research goal is to finally settle the complexity of СНеск $(\mathrm{GHD}, k)$ completely.

Research Challenge 2: Is Снеск $(\mathrm{GHD}, 2)$ tractable?

For those problems that are known to be intractable, for example, СНеск(GHD, $k$ ) for $k \geq 3$, and for those others that will turn out to be intractable, we would like to find large islands of 
tractability that correspond to meaningful restrictions of the input hypergraph instances. Ideally, such restrictions should fulfill two main criteria: (i) they need to be realistic in the sense that they apply to a large number of CQs and/or CSPs in real-life applications, and (ii) they need to be non-trivial in the sense that the restriction itself does not already imply bounded $h w, g h w$, or $f h w$. Trivial restrictions would be, for example, acyclicity or bounded treewidth. Hence, our third research problem is as follows:

Research Challenge 3: Find realistic, non-trivial restrictions on hypergraphs that entail the tractability of the СНЕск (decomp, $k$ ) problem for decomp $\in\{$ GHD, FHD $\}$.

Where we do not achieve PTIME algorithms for the precise computation of a decomposition of optimal width, we would like to find tractable methods for achieving good approximations. Note that for GHDs, the problem of approximations is solved, since $g h w(H) \leq 3 \cdot h w(H)+1$ holds for every hypergraph $H$ [4]. In contrast, for FHDs, the best known polynomial-time approximation is cubic. More precisely, in Reference [44], a polynomial-time algorithm is presented that, given a hypergraph $H$ with $f h w(H)=k$, computes an FHD of width $O\left(k^{3}\right)$. We would like to find meaningful restrictions that guarantee significantly tighter approximations in polynomial time. This leads to the fourth research problem:

Research Challenge 4: Find realistic, non-trivial restrictions on hypergraphs that allow us to compute in PTIME good approximations of $f h w(k)$.

Background and Applications. Hypergraph decompositions have meanwhile found their way into commercial database systems such as LogicBlox [6, 10, 40, 41, 48] and advanced research prototypes such as EmptyHeaded [1, 2, 50]. Moreover, since CQs and CSPs of bounded hypertree width fall into the highly parallelizable complexity class LogCFL [31], hypergraph decompositions have also been discovered as a useful tool for parallel query processing with MapReduce [5]. Hypergraph decompositions, in particular, HDs and GHDs, have been used in many other contexts, e.g., in combinatorial auctions [28] and automated selection of Web services based on recommendations from social networks [39]. Exact algorithms for computing the generalized and fractional hypertree width were published, for example, in Reference [47]; these algorithms require exponential time, which, in the light of Reference [32] and our present results cannot be improved in the general case.

CQs are the most basic and arguably the most important class of queries in the database world. Likewise, CSPs constitute one of the most fundamental classes of problems in Artificial Intelligence. Formally, CQs and CSPs are the same problem and correspond to first-order formulae using $\{\exists, \wedge\}$ but disallowing $\{\forall, \vee, \neg\}$ as connectives, which need to be evaluated over a set of finite relations: the database relations for CQs, and the constraint relations for CSPs. In practice, CQs have often fewer conjuncts (query atoms) and larger relations, while CSPs have more conjuncts but smaller relations. These problems are well-known to be NP-complete [13]. Consequently, there has been an intensive search for tractable fragments of CQs and/or CSPs over the past decades. For our work, the approaches based on decomposing the structure of a given CQ or CSP are most relevant, see, e.g., References [8, 14, 15, 17-19, 25, 31, 33, 35-38, 42, 45, 46]. The underlying structure of both is nicely captured by hypergraphs. The hypergraph $H=(V(H), E(H))$ underlying a CQ (or a CSP) $Q$ has as vertex set $V(H)$ the set of variables occurring in $Q$; moreover, for every atom in $Q, E(H)$ contains a hyperedge consisting of all variables occurring in this atom. From now on, we shall mainly talk about hypergraphs with the understanding that all our results are equally applicable to CQs and CSPs.

Main Results. First, we have investigated the above-mentioned open problem concerning the recognizability of $f h w \leq k$ for fixed $k$. Our initial hope was to find a simple adaptation of the 
NP-hardness proof in Reference [32] for recognizing $\operatorname{ghw}(H) \leq k$, for $k \geq 3$. Unfortunately, this proof dramatically fails for the fractional case. In fact, the hypergraph-gadgets in that proof are such that both "yes" and "no" instances may yield the same $f h w$. However, via crucial modifications, including the introduction of novel gadgets, we succeed to construct a reduction from 3SAT that allows us to control the $f h w$ of the resulting hypergraphs such that those hypergraphs arising from "yes" 3SAT instances have $f h w(H)=2$ and those arising from "no" instances have $f h w(H)>2$. Surprisingly, thanks to our new gadgets, the resulting proof is actually significantly simpler than the NP-hardness proof for recognizing $g h w(H) \leq k$ in Reference [32]. We thus obtain the following result:

Main Result 1: Deciding $f h w(H) \leq 2$ for hypergraphs $H$ is NP-complete and, therefore, CHeck(FHD, $k$ ) is intractable even for $k=2$.

This result can be extended to the NP-hardness of recognizing $f h w(H) \leq k$ for arbitrarily large $k$. Moreover, the same construction can be used to prove that recognizing ghw $\leq 2$ is also NP-hard, thus killing two birds with one stone.

Main Result 2: Deciding $g h w(H) \leq 2$ for hypergraphs $H$ is NP-complete and, therefore, CHeck (GHD, 2) is intractable even for $k=2$.

The Main Results 1 and 2 are presented in Section 3. These results close some smoldering open problems with bad news. We thus further concentrate on Research Challenges 3 and 4 to obtain some positive results for restricted hypergraph classes.

We first study GHDs, where we succeed to identify very general, realistic, and non-trivial restrictions that make the СНЕск $(\mathrm{GHD}, k$ ) problem tractable. These results are based on new insights about the differences of GHDs and HDs and conceptually splitting the problem into two tasks: The first problem, for a given list of possible bags of vertices and a hypergraph $H$, consists in checking whether there exists a tree decomposition of $H$ using only bags from the input. In Section 4, we show that this problem is NP-complete in general but becomes tractable when we introduce a mild restriction on the tree decompositions. The second problem consists in finding restrictions under which we only need to consider a polynomial number of possible bags.

In particular, we concentrate on the bounded intersection property (BIP), which, for a class $\mathscr{C}$ of hypergraphs requires that for some constant $i$, for each pair of distinct edges $e_{1}$ and $e_{2}$ of each hypergraph $H \in \mathscr{C},\left|e_{1} \cap e_{2}\right| \leq i$, and its generalization, the bounded multi-intersection property (BMIP), which requires that for some constant $c$ any intersection of $c$ distinct hyperedges of $H$ has at most $i$ elements for some constant $i$. A recent empirical study [23] of a large number of known CQ and CSP benchmarks showed that a high portion of instances coming from real-life applications indeed enjoys the BIP for low constant $i$ and a yet higher portion enjoys the BMIP for very low constants $c$ and $i$. We obtain the following favorable results, which are presented in Section 5:

Main Result 3: For classes of hypergraphs fulfilling the BIP or BMIP, for every constant $k$, the problem С неск $(\mathrm{GHD}, k)$ is tractable. Tractability holds even for classes $\mathscr{C}$ of hypergraphs where for some constant $c$ all intersections of $c$ distinct edges of every $H \in \mathscr{C}$ of size $n$ have $O(\log n)$ elements. Our complexity analysis reveals that for fixed $k$ and $c$ the problem Снеск $(\mathrm{GHD}, k)$ is fixed-parameter tractable parameterized by $i$ of the BMIP.

The tractability proofs for GHDs do not directly carry over to FHDs. Still, under slightly less general conditions and with some additional combinatorial insights it is possible to reduce the Снеск(FHD, $\mathrm{k}$ ) problem to the Снеск $(\mathrm{GHD}, \mathrm{k})$ scenario of the previous result. In particular, we obtain results for the BIP and a further special case of the BMIP. We then consider the degree $d$ of a 
hypergraph $H=(V(H), E(H))$, which is defined as the maximum number of hyperedges in which a vertex occurs, i.e., $d=\max _{v \in V(H)}|\{e \in E(H) \mid v \in E(H)\}|$. We say that a class $\mathscr{C}$ of hypergraphs has the bounded degree property $(B D P)$ if there exists $d \geq 1$, such that every hypergraph $H \in \mathscr{C}$ has degree $\leq d$. We obtain the following results, which are presented in Section 6:

Main Result 4: For classes of hypergraphs fulfilling either the BDP or the BIP and for every constant $k$, the problem СНEск(FHD, $k$ ) is tractable.

To get yet bigger tractable classes, we also consider approximations of an optimal FHD. Towards this goal, we study the $f h w$ in case of the BMIP and we establish an interesting connection between the BMIP and the Vapnik-Chervonenkis dimension (VC-dimension) of hypergraphs. Our research, presented in Section 6 is summarized as follows:

Main Result 5: For rather general, realistic, and non-trivial hypergraph restrictions, there exist PтIME algorithms that, for hypergraphs $H$ with $f h w(H)=k$, where $k$ is a constant, produce FHDs whose widths are significantly smaller than the best previously known approximation. In particular, the BMIP allows us to compute in polynomial time an FHD whose width is $\leq k+\epsilon$ for arbitrarily chosen constant $\epsilon>0$. Bounded VC-dimension allows us to compute in polynomial time an FHD whose width is $O(k \log k)$.

We finally turn our attention also to the optimization problem of fractional hypertree width, i.e., given a hypergraph $H$, determine $f h w(H)$ and find an FHD of width $f h w(H)$. All our algorithms for the CHeck(FHD, $k$ ) problem have a runtime exponential in the desired width $k$. Hence, even with the restrictions to the BIP or BMIP, we cannot expect an efficient approximation of $f h w$ if $f h w$ can become arbitrarily large. We will therefore study the following $K$-BoundED-FHW-Optimization problem for constant $K \geq 1$ :

\section{K-Bounded-FHW-OptimizATION}

input: $\quad$ hypergraph $H=(V, E)$;

output: if $f h w(H) \leq K$ : find an FHD $\mathcal{F}$ of $H$ with minimum width; otherwise: answer " $f h w(H)>K$."

For this bounded version of the optimization problem, we will prove the following result:

Main Result 6: There exists a polynomial time approximation scheme (PTAS; for details, see Section 6) for the K-Bounded-FHW-Optimization problem in case of the BMIP for any fixed $K \geq 1$.

\section{PRELIMINARIES}

For integers $n \geq 1$, we write $[n]$ to denote the set $\{1, \ldots, n\}$.

\subsection{Hypergraphs}

A hypergraph is a pair $H=(V(H), E(H))$, consisting of a set $V(H)$ of vertices and a set $E(H)$ of hyperedges (or, simply edges), which are non-empty subsets of $V(H)$. We assume that hypergraphs do not have isolated vertices, i.e., for each $v \in V(H)$, there is at least one edge $e \in E(H)$, s.t. $v \in e$. For a set $C \subseteq V(H)$, we define edges $(C)=\{e \in E(H) \mid e \cap C \neq \emptyset\}$ and for a set $S \subseteq E(H)$, we define $V(S)=\{v \in e \mid e \in S\}$. The rank of a hypergraph $H$ (denoted $\operatorname{rank}(H))$ is the maximum cardinality of any edge $e$ of $H$. We refer to the number of edges and vertices as $|E(H)|$ and $|V(H)|$, respectively. The size of (some reasonable representation of) $H$ will be denoted as $\|H\|$, i.e., $n \leq$ $|V(H)|+|E(H)| \cdot|V(H)|$. 
We sometimes identify sets of edges with hypergraphs. If a set of edges $E$ is used, where instead a hypergraph is expected, then we mean the hypergraph $(V, E)$, where $V$ is simply the union of all edges in $E$. For a set $S$ of edges, it is convenient to write $\cup S$ (and $\cap S$, respectively) to denote the set of vertices obtained by taking the union (or the intersection, respectively) of the edges in $S$. Hence, we can write $V(S)$ simply as $\bigcup S$.

For a hypergraph $H$ and a set $C \subseteq V(H)$, we say that a [C]-path $\pi$ from $v$ to $v^{\prime}$ consists of a sequence $v=v_{0}, \ldots, v_{h}=v^{\prime}$ of vertices and a sequence of edges $e_{0}, \ldots, e_{h-1}(h \geq 0)$ such that $\left\{v_{i}, v_{i+1}\right\} \subseteq\left(e_{i} \backslash C\right)$, for each $i \in\{0, \ldots, h-1\}$. We denote by $V(\pi)$ the set of vertices occurring in the sequence $v_{0}, \ldots, v_{h}$. Likewise, we denote by edges $(\pi)$ the set of edges occurring in the sequence $e_{0}, \ldots, e_{h-1}$. A set $W \subseteq V(H)$ of vertices is $[C]$-connected if $\forall v, v^{\prime} \in W$ there is a $[C]$-path from $v$ to $v^{\prime}$. A [C]-component is a maximal [C]-connected, non-empty set of vertices $W \subseteq V(H) \backslash C$.

The primal graph $G$ of a hypergraph $H$ is the graph with the same vertices as $H$ and an edge between vertices $v$ and $u$ iff there exists an edge $e \in E(H)$ such that $\{v, u\} \subseteq e$.

Given a hypergraph $H=\{V, E)$, the dual hypergraph $H^{d}=(W, F)$ is defined as $W=E$ and $F=\{\{e \in E \mid v \in e\} \mid v \in V\}$.

\section{2 (Fractional) Edge Covers}

Let $H=(V(H), E(H))$ be a hypergraph and consider (edge-weight) functions $\lambda: E(H) \rightarrow\{0,1\}$ and $\gamma: E(H) \rightarrow[0,1]$. For $\theta \in\{\lambda, \gamma\}$, we denote by $B(\theta)$ the set of all vertices covered by $\theta$ :

$$
B(\theta)=\left\{v \in V(H) \mid \sum_{e \in E(H), v \in e} \theta(e) \geq 1\right\} .
$$

The weight of such a function $\theta$ is defined as

$$
\text { weight }(\theta)=\sum_{e \in E(H)} \theta(e)
$$

Following Reference [31], we will sometimes consider $\lambda$ as a set with $\lambda \subseteq E(H)$ (i.e., the set of edges $e$ with $\lambda(e)=1$ ) and the weight of $\lambda$ as the cardinality of this set. However, for the sake of a uniform treatment with function $\gamma$, we shall prefer to treat $\lambda$ as a function.

Definition 2.1. An edge cover of a hypergraph $H$ is a function $\lambda: E(H) \rightarrow\{0,1\}$ such that $V(H)=B(\lambda)$. The edge cover number $\rho(H)$ is the minimum weight of all edge covers of $H$. (ILP):

Note that the edge cover number can be calculated by the following integer linear program

$$
\begin{aligned}
\text { minimize: } & \sum_{e \in E(H)} \lambda(e) \\
\text { subject to: } & \sum_{e \in E(H), v \in e} \lambda(e) \geq 1, \quad \text { for all } v \in V(H) \\
& \lambda(e) \in\{0,1\} \quad \text { for all } e \in E(H) .
\end{aligned}
$$

By substituting all $\lambda(e)$ by $\gamma(e)$ and by relaxing the last condition of the ILP above to $\gamma(e) \geq 0$, we arrive at the linear program (LP) for computing the fractional edge cover number to be defined next. Note that even though our weight function is defined to take values between 0 and 1 , we do not need to add $\gamma(e) \leq 1$ as a constraint, because implicitly by the minimization itself the weight on an edge for an edge cover is never greater than 1 . Also note that now the program above is an LP, which (in contrast to an ILP) can be solved in PTime even if $k$ is not fixed. 
Definition 2.2. A fractional edge cover of a hypergraph $H=(V(H), E(H))$ is a function $\gamma: E(H) \rightarrow$ $[0,1]$ such that $V(H)=B(\gamma)$. The fractional edge cover number $\rho^{*}(H)$ of $H$ is the minimum weight of all fractional edge covers of $H$. We write $\operatorname{supp}(\gamma)$ to denote the support of $\gamma$, i.e., $\operatorname{supp}(\gamma):=\{e \in$ $E(H) \mid \gamma(e)>0\}$.

We also extend the above definitions to subsets $S \subseteq V(H)$, i.e., an edge cover of $S$ in $H$ is a function $\lambda: E(H) \rightarrow\{0,1\}$ such that $S \subseteq B(\lambda)$. If $H$ is clear from the context, then we shall simply speak of "an edge cover of $S$ " without explicitly mentioning $H$. The edge cover number $\rho(S)$ is then the minimum weight of all edge covers of $S$. The definitions for the fractional case are extended analogously.

Clearly, we have $\rho^{*}(H) \leq \rho(H)$ for every hypergraph $H$, and $\rho^{*}(H)$ can be much smaller than $\rho(H)$. However, below, we give an example, which is important for our proof of Theorem 3.2 and where $\rho^{*}(H)$ and $\rho(H)$ coincide.

Lemma 2.3. Let $K_{2 n}$ be a clique of size $2 n$. Then the equalities $\rho\left(K_{2 n}\right)=\rho^{*}\left(K_{2 n}\right)=n$ hold.

Proof. Since we have to cover each vertex with weight $\geq 1$, the total weight on the vertices of the graph is $\geq 2 n$. As the weight of each edge adds to the weight of at most two vertices, we need at least weight $n$ on the edges to achieve $\geq 2 n$ weight on the vertices. However, we can use $n$ edges each with weight 1 to cover $2 n$ vertices. Hence, in total, we get $n \leq \rho^{*}\left(K_{2 n}\right) \leq \rho\left(K_{2 n}\right) \leq n$.

\subsection{HDs, GHDs, and FHDs}

We now define tree decompositions and three types of hypergraph decompositions:

Definition 2.4. A tree decomposition (TD) of a hypergraph $H=(V(H), E(H))$ is a tuple $\left\langle T,\left(B_{u}\right)_{u \in N(T)}\right\rangle$, such that $T=\langle N(T), E(T)\rangle$ is a rooted tree and the following conditions hold:

(1) for each $e \in E(H)$, there is a node $u \in N(T)$ with $e \subseteq B_{u}$;

(2) for each $v \in V(H)$, the set $\left\{u \in N(T) \mid v \in B_{u}\right\}$ is connected.

Definition 2.5. A generalized hypertree decomposition (GHD) of a hypergraph $H=(V(H)$, $E(H))$ is a tuple $\left\langle T,\left(B_{u}\right)_{u \in N(T)},(\lambda)_{u \in N(T)}\right\rangle$, where $\left\langle T,\left(B_{u}\right)_{u \in N(T)}\right\rangle$ is a TD of $H$ and, additionally, the following condition (3) holds:

(3) for each $u \in N(T), \lambda_{u}$ is a function $\lambda_{u}: E(H) \rightarrow\{0,1\}$ with $B_{u} \subseteq B\left(\lambda_{u}\right)$.

Let us clarify some notational conventions used throughout this article. To avoid confusion, we will consequently refer to the elements in $V(H)$ as vertices (of the hypergraph) and to the elements in $N(T)$ as the nodes of $T$ (of the decomposition). Now consider a decomposition $\mathcal{G}$ with tree structure $T$. For a node $u$ in $T$, we write $T_{u}$ to denote the subtree of $T$ rooted at $u$. By slight abuse of notation, we will often write $u^{\prime} \in T_{u}$ to denote that $u^{\prime}$ is a node in the subtree $T_{u}$ of $T$. Moreover, we define $V\left(T_{u}\right):=\bigcup_{u^{\prime} \in T_{u}} B_{u^{\prime}}$ and, for a set $V^{\prime} \subseteq V(H)$, we define nodes $\left(V^{\prime}\right)=\left\{u \in T \mid B_{u} \cap V^{\prime} \neq \emptyset\right\}$ If we want to make explicit the decomposition $\mathcal{G}$, then we also write nodes $\left(V^{\prime}, \mathcal{G}\right)$ synonymously with nodes $\left(V^{\prime}\right)$. By further overloading the nodes operator, we also write $\operatorname{nodes}\left(T_{u}\right)$ or $\operatorname{nodes}\left(T_{u}, \mathcal{G}\right)$ to denote the nodes in a subtree $T_{u}$ of $T$, i.e., $\operatorname{nodes}\left(T_{u}\right)=\operatorname{nodes}\left(T_{u}, \mathcal{G}\right)=\left\{v \mid v \in T_{u}\right\}$.

Definition 2.6. A hypertree decomposition (HD) of a hypergraph $H=(V(H), E(H))$ is a GHD, which in addition also satisfies the following condition (4):

(4) for each $u \in N(T), V\left(T_{u}\right) \cap B\left(\lambda_{u}\right) \subseteq B_{u}$.

Definition 2.7. A fractional hypertree decomposition (FHD) [35] of a hypergraph $H=(V(H)$, $E(H))$ is a tuple $\left\langle T,\left(B_{u}\right)_{u \in N(T)},(\gamma)_{u \in N(T)}\right\rangle$, where $\left\langle T,\left(B_{u}\right)_{u \in N(T)}\right\rangle$ is a TD of $H$ and, additionally, the following condition (3') holds: 
(3') for each $u \in N(T), \gamma_{u}$ is a function $\gamma_{u}: E(H) \rightarrow[0,1]$ with $B_{u} \subseteq B\left(\gamma_{u}\right)$.

The width of a GHD, HD, or FHD is the maximum weight of the functions $\lambda_{u}$ or $\gamma_{u}$, respectively, over all nodes $u$ in $T$. Moreover, the generalized hypertree width, hypertree width, and fractional hypertree width of $H$ (denoted $g h w(H), h w(H), f h w(H))$ is the minimum width over all GHDs, HDs, and FHDs of $H$, respectively. Alternatively, we could define the $g h w$ of a $\operatorname{TD}\left\langle T,\left(B_{u}\right)_{u \in N(T)}\right\rangle$ as $\max _{u \in N(T)} \rho\left(B_{u}\right)$. It is clear that the definitions lead to equivalent notions of $g h w$ for a hypergraph. The same is true for the $f h w$ of a TD, which corresponds to $\max _{u \in N(T)} \rho^{*}\left(B_{u}\right)$. Condition (2) is usually called the "connectedness condition," and condition (4) is referred to as "special condition" [31]. The set $B_{u}$ is often referred to as the "bag" at node $u$. Note that, strictly speaking, only HDs require that the underlying tree $T$ be rooted. For the sake of a uniform treatment, we assume that also the tree underlying a GHD or an FHD is rooted (with the understanding that the root is arbitrarily chosen).

We now recall two fundamental properties of the various notions of decompositions and width.

LEMMA 2.8. Let $H$ be a hypergraph and let $H^{\prime}$ be a vertex induced subhypergraph of $H$, then $h w\left(H^{\prime}\right) \leq h w(H), g h w\left(H^{\prime}\right) \leq g h w(H)$, and $f h w\left(H^{\prime}\right) \leq f h w(H)$ hold.

Lemma 2.9. Let $H$ be a hypergraph. If there exists a vertex set $S \subseteq V(H)$ such that $S$ is a clique in the primal graph, then every $H D, G H D$, or FHD of $H$ has a node u such that $S \subseteq B_{u}$.

Strictly speaking, Lemma 2.9 is a well-known property of tree decompositions-independently of the $\lambda$ - or $\gamma$-label.

\section{NP-HARDNESS}

The main result in this section is the NP-hardness of СНEск(decomp, $k$ ) with decomp $\in\{\mathrm{GHD}$, FHD and $k=2$. The proof is rather technical, we therefore begin with an informal overview of the overall strategy.

The reduction is from 3SAT. We introduce the sets $Y, Y^{\prime}$ for the literals, sets $A=\left\{a_{1}, \ldots, a_{m}\right\}$, $A^{\prime}=\left\{a_{1}^{\prime}, \ldots, a_{m}^{\prime}\right\}$, set $S$, and two special vertices $\left\{z_{1}, z_{2}\right\}$. The hypergraph $H$ to be constructed consists of three main parts: two versions of the gadget in Figure 2 (which we will refer to as $H_{0}$ and $H_{0}^{\prime}$, respectively) and a subhypergraph encoding the clauses of the 3SAT instance. The subhypergraph $H_{0}$ contains the vertices $A, Y, S, z_{1}, z_{2}$, while the subhypergraph $H_{0}^{\prime}$ contains the vertices $A^{\prime}, Y^{\prime}, S, z_{1}, z_{2}$. The intended GHD/FHD consists of a long path that connects a decomposition of $H_{0}$ at its left end and a decomposition of $H_{0}^{\prime}$ at its right end. A sketch of this decomposition is shown in Figure 1.

We assume that each of the bags $B_{1}, \ldots, B_{m}$ on the long path contains a subset of $A \cup A^{\prime}$, a subset $Y_{\text {sol }}$ of $Y \cup Y^{\prime}$ corresponding to the solution, $S$, and $z_{1}, z_{2}$. Each such bag should be covered by a pair of edges, such that one covers $z_{1}$ and there is a unique edge covering $z_{2}$ that it can be paired with and vice versa: Every edge covering $z_{1}$ covers all but a small subset of vertices of $S$, and there is a unique edge covering $z_{2}$ that fills this hole. Furthermore, every pair of edges covering $S \cup\left\{z_{1}, z_{2}\right\}$ covers exactly a subset $A_{j}^{*}=\left\{a_{1}^{\prime}, \ldots, a_{j}^{\prime}, a_{j}, \ldots, a_{m}\right\}$ of $A \cup A^{\prime}$ for some $j$. By making $a_{j}$ and $a_{j}^{\prime}$ adjacent for every $j$, we can make sure that the only way the decomposition can move from $A$ on the left to $A^{\prime}$ on the right is if bag $B_{j}$ contains exactly such a subset $A_{j}^{*}$ of $A \cup A^{\prime}$. Then, we make the following connection to the original 3SAT instance: There are exactly three pairs of edges covering $\left\{a_{1}^{\prime}, \ldots, a_{j}^{\prime}, a_{j}, \ldots, a_{m}\right\}$ corresponding to the three literals of the $j$ th clause $\left(\ell_{1} \vee \ell_{2} \vee \ell_{3}\right)$ of the formula. Each such pair fully covers $Y \cup Y^{\prime}$ except for the negation of one of the literals (i.e., $\overline{\ell_{1}}, \overline{\ell_{2}}$, or $\overline{\ell_{3}}$ ). If $Y_{\text {sol }}$ satisfies the clause, then it does not contain one of $\overline{\ell_{1}}, \overline{\ell_{2}}$, or $\overline{\ell_{3}}$, so one of the three pairs can fully cover $Y_{s o l}$ and hence all of bag $B_{j}$. In the end, the bags $B_{1}, \ldots, B_{m}$ verify that each of the $m$ clauses of the formula is satisfied by $Y_{\text {sol }}$. 
Solution path

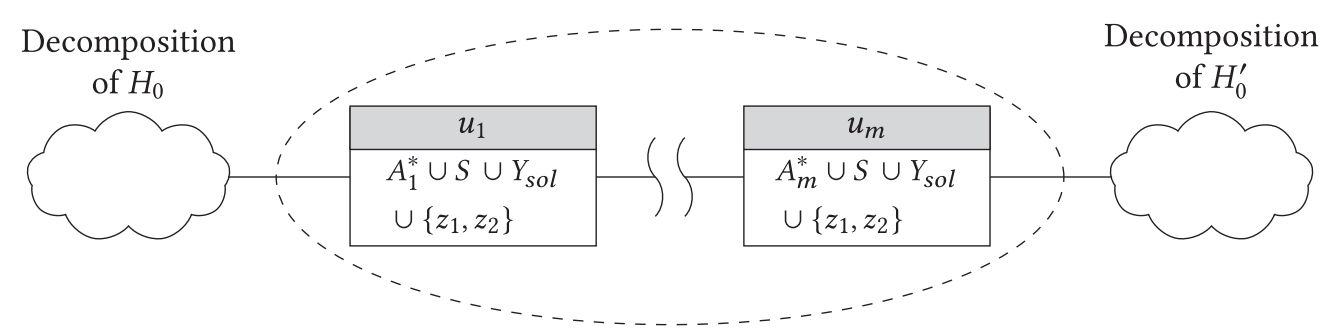

Fig. 1. Sketch of the intended GHD/FHD.

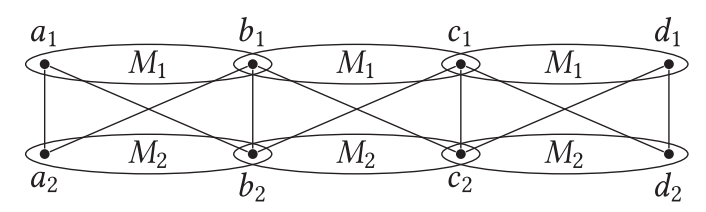

Fig. 2. Basic structure of $H_{0}$ in Lemma 3.1.

Our gadget construction makes sure that the decompositions of $H_{0}$ and $H_{0}^{\prime}$ indeed contain the specified sets of vertices (see Lemma 3.1 for the details). Moreover, we have to ensure that the same subset $Y_{\text {sol }}$ of $Y \cup Y^{\prime}$ appears in every $B_{1}, \ldots, B_{j}$ if we want to read out a satisfying assignment from the decomposition. In principle, it is possible that a $y_{i}^{\prime}$ appears or a $y_{i}$ disappears as we move from $B_{1}$ to $B_{m}$. But there is an easy standard solution for this problem: Let us repeat the path $2 n+1$ times, doing the full check of the $m$ clauses $2 n+1$ times. As there are only $2 n$ possible changes of the $y_{i}$ and $y_{i}^{\prime}$ vertices, one of the $2 n+1$ copies is free of changes, and hence can be used to deduce a satisfying assignment.

We begin by defining the aforementioned gadget (Figure 2), which will play an integral part of this construction. Its crucial properties are stated below.

LEMma 3.1. Let $M_{1}, M_{2}$ be disjoint sets and $M=M_{1} \cup M_{2}$. Let $H=(V(H), E(H))$ be a hypergraph and $H_{0}=\left(V_{0}, E_{A} \cup E_{B} \cup E_{C}\right)$ a subhypergraph of $H$ with $V_{0}=\left\{a_{1}, a_{2}, b_{1}, b_{2}, c_{1}, c_{2}, d_{1}, d_{2}\right\} \cup M$ and

$$
\begin{aligned}
& E_{A}=\left\{\left\{a_{1}, b_{1}\right\} \cup M_{1},\left\{a_{2}, b_{2}\right\} \cup M_{2},\left\{a_{1}, b_{2}\right\},\left\{a_{2}, b_{1}\right\},\left\{a_{1}, a_{2}\right\}\right\}, \\
& E_{B}=\left\{\left\{b_{1}, c_{1}\right\} \cup M_{1},\left\{b_{2}, c_{2}\right\} \cup M_{2},\left\{b_{1}, c_{2}\right\},\left\{b_{2}, c_{1}\right\},\left\{b_{1}, b_{2}\right\},\left\{c_{1}, c_{2}\right\}\right\}, \\
& E_{C}=\left\{\left\{c_{1}, d_{1}\right\} \cup M_{1},\left\{c_{2}, d_{2}\right\} \cup M_{2},\left\{c_{1}, d_{2}\right\},\left\{c_{2}, d_{1}\right\},\left\{d_{1}, d_{2}\right\}\right\},
\end{aligned}
$$

where no element from the set $R=\left\{a_{2}, b_{1}, b_{2}, c_{1}, c_{2}, d_{1}, d_{2}\right\}$ occurs in any edge of $E(H) \backslash\left(E_{A} \cup E_{B} \cup E_{C}\right)$. Then, every $F H D \mathcal{F}=\left\langle T,\left(B_{u}\right)_{u \in T},\left(\gamma_{u}\right)_{u \in T}\right\rangle$ of width $\leq 2$ of $H$ has nodes $u_{A}, u_{B}, u_{C}$ s.t.:

- $\left\{a_{1}, a_{2}, b_{1}, b_{2}\right\} \subseteq B_{u_{A}} \subseteq M \cup\left\{a_{1}, a_{2}, b_{1}, b_{2}\right\}$,

- $B_{u_{B}}=\left\{b_{1}, b_{2}, c_{1}, c_{2}\right\} \cup M$,

- $\left\{c_{1}, c_{2}, d_{1}, d_{2}\right\} \subseteq B_{u_{C}} \subseteq M \cup\left\{c_{1}, c_{2}, d_{1}, d_{2}\right\}$, and

- $u_{B}$ is on the path from $u_{A}$ to $u_{C}$.

Proof. Consider an arbitrary FHD $\mathcal{F}=\left\langle T,\left(B_{u}\right)_{u \in T},\left(\gamma_{u}\right)_{u \in T}\right\rangle$ of width $\leq 2$ of $\mathrm{H}$. Observe that $a_{1}, a_{2}, b_{1}$, and $b_{2}$ form a clique of size 4 in the primal graph. Hence, by Lemma 2.9 , there is a node $u_{A}$ in $\mathcal{F}$, such that $\left\{a_{1}, a_{2}, b_{1}, b_{2}\right\} \subseteq B_{u_{A}}$. It remains to show that also $B_{u_{A}} \subseteq M \cup\left\{a_{1}, a_{2}, b_{1}, b_{2}\right\}$ holds. To this end, we use a similar reasoning as in the proof of Lemma 2.3: to cover each vertex in $\left\{a_{1}, a_{2}, b_{1}, b_{2}\right\}$, we have to put weight $\geq 1$ on each of these four vertices. By assumption, the only 
edges containing two out of these four vertices are the edges in $E_{A} \cup\left\{\left\{b_{1}, b_{2}\right\}\right\}$. All other edges in $E(H)$ contain at most one out of these four vertices. Hence, to cover $\left\{a_{1}, a_{2}, b_{1}, b_{2}\right\}$ with weight $\leq 2$, we are only allowed to put non-zero weight on the edges in $E_{A} \cup\left\{\left\{b_{1}, b_{2}\right\}\right\}$. It follows that $B_{u_{A}} \subseteq M \cup\left\{a_{1}, a_{2}, b_{1}, b_{2}\right\}$ indeed holds.

Analogously, for the cliques $b_{1}, b_{2}, c_{1}, c_{2}$ and $c_{1}, c_{2}, d_{1}, d_{2}$, there must exist nodes $u_{B}$ and $u_{C}$ in $\mathcal{F}$ with $\left\{b_{1}, b_{2}, c_{1}, c_{2}\right\} \subseteq B_{u_{B}} \subseteq M \cup\left\{b_{1}, b_{2}, c_{1}, c_{2}\right\}$ and $\left\{c_{1}, c_{2}, d_{1}, d_{2}\right\} \subseteq B_{u_{C}} \subseteq M \cup\left\{c_{1}, c_{2}, d_{1}, d_{2}\right\}$.

It remains to show that $u_{B}$ is on the path from $u_{A}$ to $u_{C}$ and $B_{u_{B}}=\left\{b_{1}, b_{2}, c_{1}, c_{2}\right\} \cup M$ holds. We first show that $u_{B}$ is on the path between $u_{A}$ and $u_{C}$. Suppose to the contrary that it is not. We distinguish three cases. First, assume that $u_{A}$ is on the path between $u_{B}$ and $u_{C}$. Then, by connectedness, $\left\{c_{1}, c_{2}\right\} \subseteq B_{u_{A}}$, which contradicts the property $B_{u_{A}} \subseteq M \cup\left\{a_{1}, a_{2}, b_{1}, b_{2}\right\}$ shown above. Second, assume $u_{C}$ is on the path between $u_{A}$ and $u_{B}$. In this case, we have $\left\{b_{1}, b_{2}\right\} \subseteq$ $B_{u_{C}}$, which contradicts the property $B_{u_{C}} \subseteq M \cup\left\{c_{1}, c_{2}, d_{1}, d_{2}\right\}$ shown above. Third, suppose there is no path containing $u_{A}, u_{B}$, and $u_{C}$. Then there exists a node $u$ such that removing $u$ from $T$ would put $u_{A}, u_{B}, u_{C}$ in three different components. Node $u$ is therefore on the path between any pair of the three nodes. Because $u$ is on the path from $u_{A}$ to $u_{B},\left\{b_{1}, b_{s}\right\} \subseteq B_{u}$ by connectedness. Analogously, also $\left\{c_{1}, c_{2}\right\} \subseteq B_{u}$ because of the path from $u_{C}$ to $u_{B}$. Then, by the same argument as in the beginning, we have $\left\{b_{1}, b_{2}, c_{1}, c_{2}\right\} \subseteq B_{u} \subseteq M \cup\left\{b_{1}, b_{2}, c_{1}, c_{2}\right\}$. Hence, $u$ satisfies all the properties we have established for $u_{B}$ and we can just consider the node $u$, which is on the path from $u_{A}$ to $u_{C}$, to be our $u_{B}$.

We now show that also $B_{u_{B}}=\left\{b_{1}, b_{2}, c_{1}, c_{2}\right\} \cup M$ holds. Since we have already established $\left\{b_{1}, b_{2}, c_{1}, c_{2}\right\} \subseteq B_{u_{B}} \subseteq M \cup\left\{b_{1}, b_{2}, c_{1}, c_{2}\right\}$, it suffices to show $M \subseteq B_{u_{B}}$. First, let $T_{a}^{\prime}$ be the subgraph of $T$ induced by nodes $\left(\left\{a_{1}, a_{2}\right\}, \mathcal{F}\right)$ and let $T_{d}^{\prime}$ be the subgraph of $T$ induced by nodes $\left(\left\{d_{1}, d_{2}\right\}, \mathcal{F}\right)$. We show that each of the subgraphs $T_{a}^{\prime}$ and $T_{d}^{\prime}$ is connected (i.e., a subtree of $T$ ) and that the two subtrees are disjoint. The connectedness is immediate: By the connectedness condition, each of $\operatorname{nodes}\left(\left\{a_{1}\right\}, \mathcal{F}\right)$, nodes $\left(\left\{a_{2}\right\}, \mathcal{F}\right)$, nodes $\left(\left\{d_{1}\right\}, \mathcal{F}\right)$, and nodes $\left(\left\{d_{2}\right\}, \mathcal{F}\right)$ is connected. Moreover, since $H$ contains an edge $\left\{a_{1}, a_{2}\right\}$ (respectively, $\left.\left\{d_{1}, d_{2}\right\}\right)$, the two subtrees induced by nodes $\left(\left\{a_{1}\right\}, \mathcal{F}\right)$, $\operatorname{nodes}\left(\left\{a_{2}\right\}, \mathcal{F}\right)$ (respectively, nodes $\left(\left\{d_{1}\right\}, \mathcal{F}\right)$, nodes $\left.\left(\left\{d_{2}\right\}, \mathcal{F}\right)\right)$ must be connected, hence $T_{a}^{\prime}$ and $T_{d}^{\prime}$ are subtrees of $T$. It remains to show that $T_{a}^{\prime}$ and $T_{d}^{\prime}$ are disjoint.

Clearly, $u_{A} \in T_{a}^{\prime}$ and $u_{C} \in T_{d}^{\prime}$. We have established above that $u_{B}$ is on a path from $u_{A}$ to $u_{C}$. Also, $B_{u_{B}} \subseteq M \cup\left\{b_{1}, b_{2}, c_{1}, c_{2}\right\}$ and therefore $u_{B}$ is in neither $T_{a}^{\prime}$ nor $T_{d}^{\prime}$. Because both are subtrees of the tree $T$, there is only one path connecting them. But $u_{B}$ is on that path and in neither subtree, i.e., $T_{a}^{\prime}$ and $T_{d}^{\prime}$ are disjoint.

As every edge must be covered, there are nodes in $T_{a}^{\prime}$ that cover $\left\{a_{1}, b_{1}\right\} \cup M_{1}$ and $\left\{a_{2}, b_{2}\right\} \cup M_{2}$, respectively. Hence, the subtree $T_{a}^{\prime}$ covers $M=M_{1} \cup M_{2}$, i.e., $M \subseteq \cup_{u \in T_{a}^{\prime}} B_{u}$. Likewise, $T_{d}^{\prime}$ covers $M$. Since both subtrees are disjoint and $u_{B}$ is on the path between them, by the connectedness condition, we have $M \subseteq B_{u_{B}}$.

Theorem 3.2. The CHeck(decomp, $k$ ) problem is NP-complete for decomp $\in\{G H D, F H D\}$ and $k=2$.

Proof. The problem is clearly in NP: Guess a tree decomposition and check in polynomial time for each node $u$ whether $\rho\left(B_{u}\right) \leq 2$ or $\rho^{*}\left(B_{u}\right) \leq 2$, respectively, holds. The NP-hardness is proved by a reduction from 3SAT. Before presenting this reduction, we first introduce some useful notation.

Notation. For $i, j \geq 1$, we denote $\{1, \ldots, i\} \times\{1, \ldots, j\}$ by $[i ; j]$. For each $p \in[i ; j]$, we denote by $p \oplus 1$ ( $p \ominus 1$ ) the successor (predecessor) of $p$ in the usual lexicographic order on pairs, that is, the order $(1,1), \ldots,(1, j),(2,1), \ldots,(i, 1), \ldots,(i, j)$. We refer to the first element $(1,1)$ as $\min$ and to 
the last element $(i, j)$ as max. We denote by $[i ; j]^{-}$the set $[i ; j] \backslash\{\max \}$, i.e., $[i ; j]$ without the last element.

Now let $\varphi=\bigwedge_{j=1}^{m}\left(L_{j}^{1} \vee L_{j}^{2} \vee L_{j}^{3}\right)$ be an arbitrary instance of 3SAT with $m$ clauses and variables $x_{1}, \ldots, x_{n}$. From this, we will construct a hypergraph $H=(V(H), E(H))$, which consists of two copies $H_{0}, H_{0}^{\prime}$ of the (sub-)hypergraph $H_{0}$ of Lemma 3.1 plus additional edges connecting $H_{0}$ and $H_{0}^{\prime}$. We use the sets $Y=\left\{y_{1}, \ldots, y_{n}\right\}$ and $Y^{\prime}=\left\{y_{1}^{\prime}, \ldots, y_{n}^{\prime}\right\}$ to encode the truth values of the variables of $\varphi$. We denote by $Y_{\ell}\left(Y_{\ell}^{\prime}\right)$ the set $Y \backslash\left\{y_{\ell}\right\}\left(Y^{\prime} \backslash\left\{y_{\ell}^{\prime}\right\}\right)$. Furthermore, we use the sets $A=\left\{a_{p} \mid p \in[2 n+3 ; m]\right\}$ and $A^{\prime}=\left\{a_{p}^{\prime} \mid p \in[2 n+3 ; m]\right\}$, and we define the following subsets of $A$ and $A^{\prime}$, respectively:

$$
\begin{array}{ll}
A_{p}=\left\{a_{\min }, \ldots, a_{p}\right\}, & \overline{A_{p}}=\left\{a_{p}, \ldots, a_{\max }\right\}, \\
A_{p}^{\prime}=\left\{a_{\min }^{\prime}, \ldots, a_{p}^{\prime}\right\}, & \overline{A_{p}^{\prime}}=\left\{a_{p}^{\prime}, \ldots, a_{\max }^{\prime}\right\} .
\end{array}
$$

In addition, we will use another set $S$ of elements that controls and restricts the ways in which edges are combined in a possible FHD or GHD. Such a decomposition will have, implied by Lemma 3.1, two nodes $u_{B}$ and $u_{B}^{\prime}$ such that $S \subseteq B_{u_{B}}$ and $S \subseteq B_{u_{B}^{\prime}}$. From this, we will reason on the path connecting $u_{B}$ and $u_{B}^{\prime}$.

The concrete set $S$ used in our construction of $H$ is obtained as follows: Let $Q=[2 n+$ $3 ; m] \cup\{(0,1),(0,0),(1,0)\}$, hence, $Q$ is an extension of the set $[2 n+3 ; m]$ with special elements $(0,1),(0,0),(1,0)$. Then, we define the set $S$ as $S=Q \times\{1,2,3\}$.

The elements in $S$ are pairs, which we denote as $(q \mid k)$. The values $q \in Q$ are themselves pairs of integers $(i, j)$. Intuitively, $q$ indicates the position of a node on the "long" path $\pi$ in the desired FHD or GHD. The integer $k$ refers to a literal in the $j$ th clause. We will write the wildcard $*$ to indicate that a component in some element of $S$ can take an arbitrary value. For example, $(\mathrm{min} \mid *)$ denotes the set of tuples $(q \mid k)$ where $q=\min =(1,1)$ and $k$ can take an arbitrary value in $\{1,2,3\}$. We will denote by $S_{p}$ the set $(p \mid *)$. For instance, $(\min \mid *)$ will be denoted as $S_{\min }$. Further, for $p \in[2 n+3 ; m]$ and $k \in\{1,2,3\}$, we define singletons $S_{p}^{k}=\{(p \mid k)\}$. Problem reduction. Let $\varphi=\bigwedge_{j=1}^{m}\left(L_{j}^{1} \vee L_{j}^{2} \vee L_{j}^{3}\right)$ be an arbitrary instance of 3SAT with $m$ clauses and variables $x_{1}, \ldots, x_{n}$. From this, we construct a hypergraph $H=(V(H), E(H))$, that is, an instance of CHeck $($ decomp, $k)$ with decomp $\in\{\mathrm{GHD}, \mathrm{FHD}\}$ and $k=2$.

We start by defining the vertex set $V(H)$ :

$$
\begin{aligned}
V(H)= & S \cup A \cup A^{\prime} \cup Y \cup Y^{\prime} \cup\left\{z_{1}, z_{2}\right\} \cup \\
& \left\{a_{1}, a_{2}, b_{1}, b_{2}, c_{1}, c_{2}, d_{1}, d_{2}, a_{1}^{\prime}, a_{2}^{\prime}, b_{1}^{\prime}, b_{2}^{\prime}, c_{1}^{\prime}, c_{2}^{\prime}, d_{1}^{\prime}, d_{2}^{\prime}\right\} .
\end{aligned}
$$

The edges of $H$ are defined in three steps. First, we take two copies of the subhypergraph $H_{0}$ used in Lemma 3.1:

- Let $H_{0}=\left(V_{0}, E_{0}\right)$ be the hypergraph of Lemma 3.1 with $V_{0}=\left\{a_{1}, a_{2}, b_{1}, b_{2}, c_{1}, c_{2}, d_{1}, d_{2}\right\} \cup$ $M_{1} \cup M_{2}$ and $E_{0}=E_{A} \cup E_{B} \cup E_{C}$, where we set $M_{1}=S \backslash S_{(0,1)} \cup\left\{z_{1}\right\}$ and $M_{2}=Y \cup S_{(0,1)} \cup\left\{z_{2}\right\}$.

- Let $H_{0}^{\prime}=\left(V_{0}^{\prime}, E_{0}^{\prime}\right)$ be the corresponding hypergraph, with $V_{0}^{\prime}=\left\{a_{1}^{\prime}, a_{2}^{\prime}, b_{1}^{\prime}, b_{2}^{\prime}, c_{1}^{\prime}, c_{2}^{\prime}, d_{1}^{\prime}, d_{2}^{\prime}\right\} \cup$ $M_{1}^{\prime} \cup M_{2}^{\prime}$ and $E_{A}^{\prime}, E_{B}^{\prime}, E_{C}^{\prime}$ are the primed versions of the edge sets $M_{1}^{\prime}=S \backslash S_{(1,0)} \cup\left\{z_{1}\right\}$ and $M_{2}^{\prime}=Y^{\prime} \cup S_{(1,0)} \cup\left\{z_{2}\right\}$.

In the second step, we define the edges that (as we will see) enforce the existence of a "long" path $\pi$ between the nodes covering $H_{0}$ and the nodes covering $H_{0}^{\prime}$ in any FHD of width $\leq 2$.

- $e_{p}=A_{p}^{\prime} \cup \overline{A_{p}}$, for $p \in[2 n+3 ; m]^{-}$,

- $e_{y_{i}}=\left\{y_{i}, y_{i}^{\prime}\right\}$, for $1 \leq i \leq n$, 
- For $p=(i, j) \in[2 n+3 ; m]^{-}$and $k \in\{1,2,3\}$ :

$$
\begin{aligned}
& e_{p}^{k, 0}= \begin{cases}\overline{A_{p}} \cup\left(S \backslash S_{p}^{k}\right) \cup Y \cup\left\{z_{1}\right\} & \text { if } L_{j}^{k}=x_{\ell} \\
\overline{A_{p}} \cup\left(S \backslash S_{p}^{k}\right) \cup Y_{\ell} \cup\left\{z_{1}\right\} & \text { if } L_{j}^{k}=\neg x_{\ell},\end{cases} \\
& e_{p}^{k, 1}= \begin{cases}A_{p}^{\prime} \cup S_{p}^{k} \cup Y_{\ell}^{\prime} \cup\left\{z_{2}\right\} & \text { if } L_{j}^{k}=x_{\ell} \\
A_{p}^{\prime} \cup S_{p}^{k} \cup Y^{\prime} \cup\left\{z_{2}\right\} & \text { if } L_{j}^{k}=\neg x_{\ell} .\end{cases}
\end{aligned}
$$

Finally, we need edges that connect $H_{0}$ and $H_{0}^{\prime}$ with the above edges covered by the nodes of the "long" path $\pi$ in a GHD or FHD:

- $e_{(0,0)}^{0}=\left\{a_{1}\right\} \cup A \cup S \backslash S_{(0,0)} \cup Y \cup\left\{z_{1}\right\}$,

- $e_{(0,0)}^{1}=S_{(0,0)} \cup Y^{\prime} \cup\left\{z_{2}\right\}$,

- $e_{\max }^{0}=S \backslash S_{\max } \cup Y \cup\left\{z_{1}\right\}$,

- $e_{\max }^{1}=\left\{a_{1}^{\prime}\right\} \cup A^{\prime} \cup S_{\max } \cup Y^{\prime} \cup\left\{z_{2}\right\}$.

This concludes the construction of the hypergraph $H$. Before we prove the correctness of the problem reduction, we give an example that will help to illustrate the intuition underlying this construction.

Example 3.3. Suppose that an instance of 3SAT is given by the propositional formula $\varphi=\left(x_{1} \vee\right.$ $\left.\neg x_{2} \vee x_{3}\right) \wedge\left(\neg x_{1} \vee x_{2} \vee \neg x_{3}\right)$, i.e.: We have $n=3$ variables and $m=2$ clauses. From this, we construct a hypergraph $H=(V(H), E(H))$. First, we instantiate the sets $Q, A, A^{\prime}, S, Y$, and $Y^{\prime}$ from our problem reduction.

$$
\begin{aligned}
A & =\left\{a_{(1,1)}, a_{(1,2)}, a_{(2,1)}, a_{(2,2)}, \ldots, a_{(9,1)}, a_{(9,2)}\right\} \\
A^{\prime} & =\left\{a_{(1,1)}^{\prime}, a_{(1,2)}^{\prime}, a_{(2,1)}^{\prime}, a_{(2,2)}^{\prime}, \ldots, a_{(9,1)}^{\prime}, a_{(9,2)}^{\prime}\right\} \\
Q & =\{(1,1),(1,2),(2,1),(2,2), \ldots,(9,1),(9,2)\} \cup\{(0,1),(0,0),(1,0)\}, \\
S & =Q \times\{1,2,3\}, \\
Y & =\left\{y_{1}, y_{2}, y_{3}\right\}, \\
Y^{\prime} & =\left\{y_{1}^{\prime}, y_{2}^{\prime}, y_{3}^{\prime}\right\} .
\end{aligned}
$$

According to our problem reduction, the set $V(H)$ of vertices of $H$ is

$$
\begin{aligned}
V(H)= & S \cup A \cup A^{\prime} \cup Y \cup Y^{\prime} \cup\left\{z_{1}, z_{2}\right\} \cup \\
& \left\{a_{1}, a_{2}, b_{1}, b_{2}, c_{1}, c_{2}, d_{1}, d_{2}\right\} \cup\left\{a_{1}^{\prime}, a_{2}^{\prime}, b_{1}^{\prime}, b_{2}^{\prime}, c_{1}^{\prime}, c_{2}^{\prime}, d_{1}^{\prime}, d_{2}^{\prime}\right\} .
\end{aligned}
$$

The set $E(H)$ of edges of $H$ is defined in several steps. First, the edges in $H_{0}$ and $H_{0}^{\prime}$ are defined: We thus have the subsets $E_{A}, E_{B}, E_{C}, E_{A}^{\prime}, E_{B}^{\prime}, E_{C}^{\prime} \subseteq E(H)$, whose definition is based on the sets $M_{1}=S \backslash S_{(0,1)} \cup\left\{z_{1}\right\}, M_{2}=Y \cup S_{(0,1)} \cup\left\{z_{2}\right\}, M_{1}^{\prime}=S \backslash S_{(1,0)} \cup\left\{z_{1}\right\}$, and $M_{2}^{\prime}=Y^{\prime} \cup S_{(1,0)} \cup\left\{z_{2}\right\}$. The definition of the edges

$$
\begin{array}{rlrl}
e_{p} & =A_{p}^{\prime} \cup \overline{A_{p}} & & \text { for } p \in\{(1,1),(1,2), \ldots(8,1),(8,2),(9,1)\}, \\
e_{y_{i}} & =\left\{y_{i}, y_{i}^{\prime}\right\} & \text { for } 1 \leq i \leq 3, \\
e_{(0,0)}^{0} & =\left\{a_{1}\right\} \cup A \cup S \backslash S_{(0,0)} \cup Y \cup\left\{z_{1}\right\}, \\
e_{(0,0)}^{1} & =S_{(0,0)} \cup Y^{\prime} \cup\left\{z_{2}\right\}, & \\
e_{(9,2)}^{0} & =S \backslash S_{(9,2)} \cup Y \cup\left\{z_{1}\right\}, & \text { and } \\
e_{(9,2)}^{1} & =\left\{a_{1}^{\prime}\right\} \cup A^{\prime} \cup S_{(9,2)} \cup Y^{\prime} \cup\left\{z_{2}\right\}
\end{array}
$$


is straightforward. We concentrate on the edges $e_{p}^{k, 0}$ and $e_{p}^{k, 1}$ for $p \in\{(1,1),(1,2), \ldots(8,1),(8,2)$, $(9,1)\}$ and $k \in\{1,2,3\}$. These edges play the key role for covering the bags of the nodes along the "long" path $\pi$ in any FHD or GHD of $H$. This path can be thought of as being structured in nine blocks. Consider an arbitrary $i \in\{1, \ldots, 9\}$. Then $e_{(i, 1)}^{k, 0}$ and $e_{(i, 1)}^{k, 1}$ encode the $k$ th literal of the first clause and $e_{(i, 2)}^{k, 0}$ and $e_{(i, 2)}^{k, 1}$ encode the $k$ th literal of the second clause (the latter is only defined for $i \leq 8)$. These edges are defined as follows: The edges $e_{(i, 1)}^{1,0}$ and $e_{(i, 1)}^{1,1}$ encode the first literal of the first clause, i.e., the positive literal $x_{1}$. We thus have

$$
\begin{aligned}
e_{(i, 1)}^{1,0} & =\overline{A_{(i, 1)}} \cup\left(S \backslash S_{(i, 1)}^{1}\right) \cup\left\{y_{1}, y_{2}, y_{3}\right\} \cup\left\{z_{1}\right\} \text { and } \\
e_{(i, 1)}^{1,1} & =A_{(i, 1)}^{\prime} \cup S_{(i, 1)}^{1} \cup\left\{y_{2}^{\prime}, y_{3}^{\prime}\right\} \cup\left\{z_{2}\right\} .
\end{aligned}
$$

The edges $e_{(i, 1)}^{2,0}$ and $e_{(i, 1)}^{2,1}$ encode the second literal of the first clause, i.e., the negative literal $\neg x_{2}$. Likewise, $e_{(i, 1)}^{3,0}$ and $e_{(i, 1)}^{3,1}$ encode the third literal of the first clause, i.e., the positive literal $x_{3}$. Hence,

$$
\begin{aligned}
e_{(i, 1)}^{2,0} & =\overline{A_{(i, 1)}} \cup\left(S \backslash S_{(i, 1)}^{2}\right) \cup\left\{y_{1}, y_{3}\right\} \cup\left\{z_{1}\right\}, \\
e_{(i, 1)}^{2,1} & =A_{(i, 1)}^{\prime} \cup S_{(i, 1)}^{2} \cup\left\{y_{1}^{\prime}, y_{2}^{\prime}, y_{3}^{\prime}\right\} \cup\left\{z_{2}\right\}, \\
e_{(i, 1)}^{3,0} & =\overline{A_{(i, 1)}} \cup\left(S \backslash S_{(i, 1)}^{3}\right) \cup\left\{y_{1}, y_{2}, y_{3}\right\} \cup\left\{z_{1}\right\}, \text { and } \\
e_{(i, 1)}^{3,1} & =A_{(i, 1)}^{\prime} \cup S_{(i, 1)}^{3} \cup\left\{y_{1}^{\prime}, y_{2}^{\prime}\right\} \cup\left\{z_{2}\right\} .
\end{aligned}
$$

Analogously, the edges $e_{(i, 2)}^{1,0}$ and $e_{(i, 2)}^{1,1}$ (encoding the first literal of the second clause, i.e., $\left.\neg x_{1}\right)$, the edges $e_{(i, 2)}^{2,0}$ and $e_{(i, 2)}^{2,1}$ (encoding the second literal of the second clause, i.e., $x_{2}$ ), and the edges $e_{(i, 2)}^{3,0}$ and $e_{(i, 2)}^{3,1}$ (encoding the third literal of the second clause, i.e., $\left.\neg x_{3}\right)$ are defined as follows:

$$
\begin{aligned}
e_{(i, 2)}^{1,0} & =\overline{A_{(i, 2)}} \cup\left(S \backslash S_{(i, 2)}^{1}\right) \cup\left\{y_{2}, y_{3}\right\} \cup\left\{z_{1}\right\}, \\
e_{(i, 2)}^{1,1} & =A_{(i, 2)}^{\prime} \cup S_{(i, 2)}^{1} \cup\left\{y_{1}^{\prime}, y_{2}^{\prime}, y_{3}^{\prime}\right\} \cup\left\{z_{2}\right\}, \\
e_{(i, 2)}^{2,0} & =\overline{A_{(i, 2)}} \cup\left(S \backslash S_{(i, 2)}^{2}\right) \cup\left\{y_{1}, y_{2}, y_{3}\right\} \cup\left\{z_{1}\right\}, \\
e_{(i, 2)}^{2,1} & =A_{(i, 2)}^{\prime} \cup S_{(i, 2)}^{2} \cup\left\{y_{1}^{\prime}, y_{3}^{\prime}\right\} \cup\left\{z_{2}\right\}, \\
e_{(i, 2)}^{3,0} & =\overline{A_{(i, 2)}} \cup\left(S \backslash S_{(i, 2)}^{3}\right) \cup\left\{y_{1}, y_{2}\right\} \cup\left\{z_{1}\right\}, \text { and } \\
e_{(i, 2)}^{3,1} & =A_{(i, 2)}^{\prime} \cup S_{(i, 2)}^{3} \cup\left\{y_{1}^{\prime}, y_{2}^{\prime}, y_{3}^{\prime}\right\} \cup\left\{z_{2}\right\} .
\end{aligned}
$$

The crucial property of these pairs of edges $e_{(i, j)}^{k, 0}$ and $e_{(i, j)}^{k, 1}$ is that they together encode the $k$ th literal of the $j$ th clause in the following way: If the literal is of the form $x_{\ell}$ (respectively, of the form $\left.\neg x_{\ell}\right)$, then $e_{(i, j)}^{k, 0} \cup e_{(i, j)}^{k, 1}$ covers all of $Y \cup Y^{\prime}$ except for $y_{\ell}^{\prime}$ (respectively, except for $y_{\ell}$ ).

Formula $\varphi$ in this example is clearly satisfiable, e.g., by the truth assignment $\sigma$ with $\sigma\left(x_{1}\right)=$ true and $\sigma\left(x_{2}\right)=\sigma\left(x_{3}\right)=$ false. Hence, for the problem reduction to be correct, there must exist a GHD (and thus also an FHD) of width 2 of $H$. In Figure 3, the tree structure $T$ plus the bags $\left(B_{t}\right)_{t \in T}$ of such a GHD is displayed. Moreover, in Table 1, the precise definition of $B_{u}$ and $\lambda_{u}$ of every node $u \in T$ is given: In the column labelled $B_{u}$, the set of vertices contained in $B_{u}$ for each node $u \in T$ is shown. In the column labelled $\lambda_{u}$, the two edges with weight 1 are shown. For the row with label $u_{p \in[2 n+3 ; m]^{-}}$, the entry in the last column is $e_{p}^{k_{p}, 0}, e_{p}^{k_{p}, 1}$. By this, we mean that, for every $p$, an appropriate value $k_{p} \in\{1,2,3\}$ has to be determined. It will be explained below how to find an appropriate value $k_{p}$ for each $p$. The set $Z$ in the bags of this GHD is defined as 
$Z=\left\{y_{i} \mid \sigma\left(x_{i}\right)=\right.$ true $\} \cup\left\{y_{i}^{\prime} \mid \sigma\left(x_{i}\right)=\right.$ false $\}$. In this example, for the chosen truth assignment $\sigma$, we thus have $Z=\left\{y_{1}, y_{2}^{\prime}, y_{3}^{\prime}\right\}$. The bags $B_{t}$ and the edge covers $\lambda_{t}$ for each $t \in T$ are explained below.

The nodes $u_{C}, u_{B}, u_{A}$ to cover the edges of the subhypergraph $H_{0}$ and the nodes $u_{A}^{\prime}, u_{B}^{\prime}, u_{C}^{\prime}$ to cover the edges of the subhypergraph $H_{0}^{\prime}$ are clear by Lemma 3.1. The purpose of the nodes $u_{\min } \ominus 1$ and $u_{\max }$ is mainly to make sure that each edge $\left\{y_{i}, y_{i}^{\prime}\right\}$ is covered by some bag. Recall that the set $Z$ contains exactly one of $y_{i}$ and $y_{i}^{\prime}$ for every $i$. Hence, the node $u_{\min \ominus 1}$ (respectively, $u_{\max }$ ) covers each edge $\left\{y_{i}, y_{i}^{\prime}\right\}$, such that $y_{i}^{\prime} \in Z$ (respectively, $y_{i} \in Z$ ).

We now have a closer look at the nodes $u_{(1,1)}$ to $u_{(9,1)}$ on the "long" path $\pi$. More precisely, let us look at the nodes $u_{(i, 1)}$ and $u_{(i, 2)}$ for some $i \in\{1, \ldots, 8\}$, i.e., the "ith block." It will turn out that the bags at these nodes can be covered by edges from $H$ because $\varphi$ is satisfiable. Indeed, our choice of $\lambda_{u_{(i, 1)}}$ and $\lambda_{u_{(i, 2)}}$ is guided by the literals satisfied by the truth assignment $\sigma$, namely: For $\lambda_{u_{(i, j)}}$, we have to choose some $k_{j}$, such that the $k_{j}$ th literal in the $j$ th clause is true in $\sigma$. For instance, we may define $\lambda_{u_{(i, 1)}}$ and $\lambda_{u_{(i, 2)}}$ as follows:

$$
\lambda_{u_{(i, 1)}}=\left\{e_{(i, 1)}^{1,0}, e_{(i, 1)}^{1,1}\right\}, \quad \lambda_{u_{(i, 2)}}=\left\{e_{(i, 2)}^{3,0}, e_{(i, 2)}^{3,1}\right\}
$$

The covers $\lambda_{u_{(i, 1)}}$ and $\lambda_{u_{(i, 2)}}$ were chosen because the first literal of the first clause and the third literal of the second clause are true in $\sigma$. Now let us verify that $\lambda_{u_{(i, 1)}}$ and $\lambda_{u_{(i, 2)}}$ are indeed covers of $B_{u_{(i, 1)}}$ and $B_{u_{(i, 2)}}$, respectively. By the definition of the edges $e_{(i, j)}^{k, 0}, e_{(i, j)}^{k, 1}$ for $j \in\{1,2\}$ and $k \in\{1,2,3\}$, it is immediate that $e_{(i, j)}^{k, 0} \cup e_{(i, j)}^{k, 1}$ covers $\overline{A_{(i, j)}} \cup A_{(i, j)}^{\prime} \cup S \cup\left\{z_{1}, z_{2}\right\}$. The only non-trivial question is if $\lambda_{u_{(i, j)}}$ also covers $Z$. Recall that by definition, $\left(e_{(i, 1)}^{1,0} \cup e_{(i, 1)}^{1,1}\right) \supseteq\left(Y \cup Y^{\prime}\right) \backslash\left\{y_{1}^{\prime}\right\}$. Our truth assignment $\sigma$ sets $\sigma\left(x_{1}\right)=$ true. Hence, by our definition of $Z$, we have $y_{1} \in Z$ and $y_{1}^{\prime} \notin Z$. This means that $e_{(i, 1)}^{1,0} \cup e_{(i, 1)}^{1,1}$ indeed covers $Z$ and, hence, all of $B_{u_{(i, 1)}}$. Note that we could have also chosen $\lambda_{u_{(i, 1)}}=\left\{e_{(i, 1)}^{2,0}, e_{(i, 1)}^{2,1}\right\}$, since also the second literal of the first clause (i.e., $\left.\neg x_{2}\right)$ is true in $\sigma$. In this case, we would have $\left(e_{(i, 1)}^{2,0} \cup e_{(i, 1)}^{2,1}\right) \supseteq\left(Y \cup Y^{\prime}\right) \backslash\left\{y_{2}\right\}$ and $Z$ indeed does not contain $y_{2}$. Conversely, setting $\lambda_{u_{(i, 1)}}=\left\{e_{(i, 1)}^{3,0}, e_{(i, 1)}^{3,1}\right\}$ would fail, because in this case, $y_{3}^{\prime} \notin\left(e_{(i, 1)}^{3,0} \cup e_{(i, 1)}^{3,1}\right)$, since $x_{3}$ occurs positively in the first clause. However, we have $y_{3}^{\prime} \in Z$ by definition of $Z$, because $\sigma\left(x_{3}\right)=$ false holds.

Checking that $\lambda_{u_{(i, 2)}}$ as defined above covers $Z$ is done analogously. Note that in the second clause, only the third literal is satisfied by $\sigma$. Hence, setting $\lambda_{u_{(i, 2)}}=\left\{e_{(i, 2)}^{3,0}, e_{(i, 2)}^{3,1}\right\}$ is the only option to cover $B_{u_{(i, 2)}}$ (in particular, to cover $Z$ ). Finally, note that $\sigma$ as defined above is not the only satisfying truth assignment of $\varphi$. For instance, we could have chosen $\sigma\left(x_{1}\right)=\sigma\left(x_{2}\right)=\sigma\left(x_{3}\right)=$ true. In this case, we would define $Z=\left\{y_{1}, y_{2}, y_{3}\right\}$ and the covers $\lambda_{u_{(i, j)}}$ would have to be chosen according to an arbitrary choice of one literal per clause that is satisfied by this assignment $\sigma$. $\diamond$

To prove the correctness of our problem reduction, we have to show the two equivalences: first, that $g h w(H) \leq 2$ if and only if $\varphi$ is satisfiable and, second, that $f h w(H) \leq 2$ if and only if $\varphi$ is satisfiable. We prove the two directions of these equivalences separately.

Proof of the "if"-direction. First assume that $\varphi$ is satisfiable. It suffices to show that then $H$ has a GHD of width $\leq 2$, because $f h w(H) \leq g h w(H)$ holds. Let $\sigma$ be a satisfying truth assignment. Let us fix for each $j \leq m$, some $k_{j} \in\{1,2,3\}$ such that $\sigma\left(L_{j}^{k_{j}}\right)=1$. By $\ell_{j}$, we denote the index of the variable in the literal $L_{j}^{k_{j}}$, that is, $L_{j}^{k_{j}}=x_{\ell_{j}}$ or $L_{j}^{k_{j}}=\neg x_{\ell_{j}}$. For $p=(i, j)$, let $k_{p}$ refer to $k_{j}$ and let $L_{p}^{k_{p}}$ refer to $L_{j}^{k_{j}}$. Finally, we define $Z$ as $Z=\left\{y_{i} \mid \sigma\left(x_{i}\right)=1\right\} \cup\left\{y_{i}^{\prime} \mid \sigma\left(x_{i}\right)=0\right\}$.

A GHD $\mathcal{G}=\left\langle T,\left(B_{u}\right)_{u \in T},\left(\lambda_{u}\right)_{u \in T}\right\rangle$ of width 2 for $H$ is constructed as follows: $T$ is a path $u_{C}$, $u_{B}, u_{A}, u_{\min \ominus 1}, u_{\min }, \ldots, u_{\max }, u_{A}^{\prime}, u_{B}^{\prime}, u_{C}^{\prime}$. The construction is illustrated in Figure 3 . The precise 


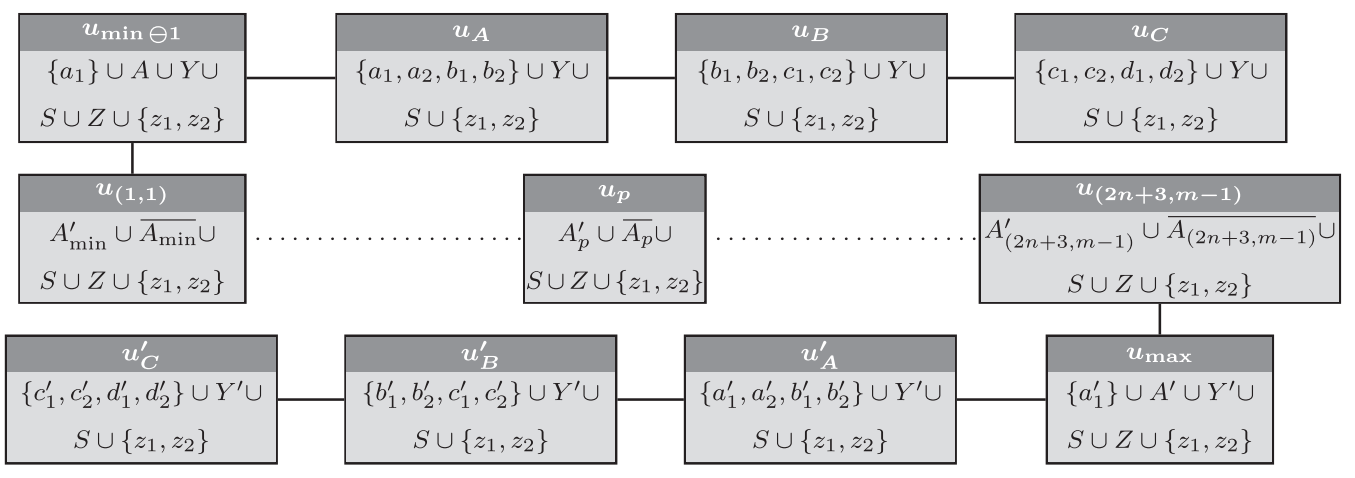

Fig. 3. Intended path of the GHD of hypergraph $H$ in the proof of Theorem 3.2.

Table 1. Definition of $B_{u}$ and $\lambda_{u}$ for GHD of $H$

\begin{tabular}{|c|c|c|}
\hline$u \in T$ & $B_{u}$ & $\lambda_{u}$ \\
\hline$u_{C}$ & $\left\{d_{1}, d_{2}, c_{1}, c_{2}\right\} \cup Y \cup S \cup\left\{z_{1}, z_{2}\right\}$ & $\left\{c_{1}, d_{1}\right\} \cup M_{1},\left\{c_{2}, d_{2}\right\} \cup M_{2}$ \\
$u_{B}$ & $\left\{c_{1}, c_{2}, b_{1}, b_{2}\right\} \cup Y \cup S \cup\left\{z_{1}, z_{2}\right\}$ & $\left\{b_{1}, c_{1}\right\} \cup M_{1},\left\{b_{2}, c_{2}\right\} \cup M_{2}$ \\
$u_{A}$ & $\left\{b_{1}, b_{2}, a_{1}, a_{2}\right\} \cup Y \cup S \cup\left\{z_{1}, z_{2}\right\}$ & $\left\{a_{1}, b_{1}\right\} \cup M_{1},\left\{a_{2}, b_{2}\right\} \cup M_{2}$ \\
$u_{\min \ominus 1}$ & $\left\{a_{1}\right\} \cup A \cup Y \cup S \cup Z \cup\left\{z_{1}, z_{2}\right\}$ & $e_{(0,0)}^{0}, e_{(0,0)}^{1}$ \\
$u_{p \in[2 n+3 ; m]^{-}}$ & $A_{p}^{\prime} \cup \overline{A_{p}} \cup S \cup Z \cup\left\{z_{1}, z_{2}\right\}$ & $e_{p}^{k_{p}, 0}, e_{p}^{k_{p}, 1}$ \\
$u_{\max }$ & $\left\{a_{1}^{\prime}\right\} \cup A^{\prime} \cup Y^{\prime} \cup S \cup Z \cup\left\{z_{1}, z_{2}\right\}$ & $e_{\max }^{0}, e_{\max }^{1}$ \\
$u_{A}^{\prime}$ & $\left\{a_{1}^{\prime}, a_{2}^{\prime}, b_{1}^{\prime}, b_{2}^{\prime}\right\} \cup Y^{\prime} \cup S \cup\left\{z_{1}, z_{2}\right\}$ & $\left\{a_{1}^{\prime}, b_{1}^{\prime}\right\} \cup M_{1}^{\prime},\left\{a_{2}^{\prime}, b_{2}^{\prime}\right\} \cup M_{2}^{\prime}$ \\
$u_{B}^{\prime}$ & $\left\{b_{1}^{\prime}, b_{2}^{\prime}, c_{1}^{\prime}, c_{2}^{\prime}\right\} \cup Y^{\prime} \cup S \cup\left\{z_{1}, z_{2}\right\}$ & $\left\{b_{1}^{\prime}, c_{1}^{\prime}\right\} \cup M_{1}^{\prime},\left\{b_{2}^{\prime}, c_{2}^{\prime}\right\} \cup M_{2}^{\prime}$ \\
$u_{C}^{\prime}$ & $\left\{c_{1}^{\prime}, c_{2}^{\prime}, d_{1}^{\prime}, d_{2}^{\prime}\right\} \cup Y^{\prime} \cup S \cup\left\{z_{1}, z_{2}\right\}$ & $\left\{c_{1}^{\prime}, d_{1}^{\prime}\right\} \cup M_{1}^{\prime},\left\{c_{2}^{\prime}, d_{2}^{\prime}\right\} \cup M_{2}^{\prime}$ \\
\hline
\end{tabular}

definition of $B_{u}$ and $\lambda_{u}$ is given in Table 1 . Clearly, the GHD has width $\leq 2$. We now show that $\mathcal{G}$ is indeed a GHD of $H$ :

(1) For each edge $e \in E$, there is a node $u \in T$, such that $e \subseteq B_{u}$ :

- $\forall e \in E_{X}: e \subseteq B_{u_{X}}$ for all $X \in\{A, B, C\}$,

- $\forall e^{\prime} \in E_{X}^{\prime}: e^{\prime} \subseteq B_{u_{X}^{\prime}}$ for all $X \in\{A, B, C\}$,

- $e_{p} \subseteq B_{u_{p}}$ for $p \in[2 n+3 ; m]^{-}$,

- $e_{y_{i}} \subseteq B_{u_{\min \ominus 1}}$ (if $y_{i}^{\prime} \in Z$ ) or $e_{y_{i}} \subseteq B_{u_{\max }}$ (if $y_{i} \in Z$ ), respectively,

- $e_{p}^{k, 0} \subseteq B_{u_{\min \ominus 1}}$ for $p \in[2 n+3 ; m]^{-}$,

- $e_{p}^{k, 1} \subseteq B_{u_{\max }}$ for $p \in[2 n+3 ; m]^{-}$,

- $e_{(0,0)}^{0} \subseteq B_{u_{\min \ominus 1}}, e_{(0,0)}^{1} \subseteq B_{u_{\max }}$,

- $e_{\max }^{0} \subseteq B_{u_{\min \ominus 1}}$ and $e_{\max }^{1} \subseteq B_{u_{\max }}$.

All of the above inclusions can be verified in Table 1 .

(2) For each vertex $v \in V$, the set $\left\{u \in T \mid v \in B_{u}\right\}$ induces a connected subtree of $T$, which is easy to verify in Table 1 .

(3) For each $u \in T, B_{u} \subseteq B\left(\lambda_{u}\right)$ : The only inclusion that cannot be easily verified in Table 1 is $B_{u_{p}} \subseteq B\left(\lambda_{u_{p}}\right)$. In fact, this is the only place in the proof where we make use of the assumption that $\varphi$ is satisfiable. First, notice that the set $A_{p}^{\prime} \cup \overline{A_{p}} \cup S \cup\left\{z_{1}, z_{2}\right\}$ is clearly a subset of $B\left(\lambda_{u_{p}}\right)$. It remains to show that $Z \subseteq B\left(\lambda_{u_{p}}\right)$ holds for arbitrary $p \in[2 n+3 ; m]^{-}$. We show this property by a case distinction on the form of $L_{p}^{k_{p}}$. 
Case (1): First, assume that $L_{p}^{k_{p}}=x_{\ell_{j}}$ holds. Then $\sigma\left(x_{\ell_{j}}\right)=1$ and, therefore, $y_{\ell_{j}}^{\prime} \notin Z$. But, by definition of $e_{p}^{k_{p}, 0}$ and $e_{p}^{k_{p}, 1}$, vertex $y_{\ell_{j}}^{\prime}$ is the only element of $Y \cup Y^{\prime}$ not contained in $B\left(\lambda_{u_{p}}\right)$. Since $Z \subseteq\left(Y \cup Y^{\prime}\right)$ and $y_{\ell_{j}}^{\prime} \notin Z$, we have that $Z \subseteq B\left(\lambda_{u_{p}}\right)$.

Case (2): Now assume that $L_{p}^{k_{p}}=\neg x_{\ell_{j}}$ holds. Then $\sigma\left(x_{\ell_{j}}\right)=0$ and, therefore, $y_{\ell_{j}} \notin Z$. But, by definition of $e_{p}^{k_{p}, 0}$ and $e_{p}^{k_{p}, 1}$, vertex $y_{\ell_{j}}$ is the only element of $Y \cup Y^{\prime}$ not contained in $B\left(\lambda_{u_{p}}\right)$. Since $Z \subseteq\left(Y \cup Y^{\prime}\right)$ and $y_{\ell_{j}} \notin Z$, we have that $Z \subseteq B\left(\lambda_{u_{p}}\right)$.

Two crucial lemmas. Before we prove the "only if'-direction, we define the notion of complementary edges and state two important lemmas related to this notion.

Definition 3.4. Let $e$ and $e^{\prime}$ be two edges from the hypergraph $H$ as defined before. We say $e^{\prime}$ is the complementary edge of $e$ (or, simply, $e, e^{\prime}$ are complementary edges) whenever

- $e \cap S=S \backslash S^{\prime}$ for some $S^{\prime} \subseteq S$ and

- $e^{\prime} \cap S=S^{\prime}$.

Observe that for every edge in our construction that covers $S \backslash S^{\prime}$ for some $S^{\prime} \subseteq S$ there is a complementary edge that covers $S^{\prime}$, for example, $e_{p}^{k, 0}$ and $e_{p}^{k, 1}, e_{(0,0)}^{0}$, and $e_{(0,0)}^{1}$, and so on. In particular, there is no edge that covers $S$ completely. Moreover, consider arbitrary subsets $S_{1}, S_{2}$ of $S$, s.t. (syntactically) $S \backslash S_{i}$ is part of the definition of $e_{i}$ for some $e_{i} \in E(H)$ with $i \in\{1,2\}$. Then $S_{1}$ and $S_{2}$ are disjoint.

We now present two lemmas needed for the "only if"-direction.

LEMMA 3.5. Let $\mathcal{F}=\left\langle T,\left(B_{u}\right)_{u \in T},\left(\gamma_{u}\right)_{u \in T}\right\rangle$ be an FHD of width $\leq 2$ of the hypergraph $H$ constructed above. For every node $u$ with $S \cup\left\{z_{1}, z_{2}\right\} \subseteq B_{u}$ and every pair e, $e^{\prime}$ of complementary edges, it holds that $\gamma_{u}(e)=\gamma_{u}\left(e^{\prime}\right)$.

Proof. First, we try to cover $z_{1}$ and $z_{2}$. For $z_{1}$, we have to put total weight 1 on the edges in $E^{0}$, and to cover $z_{2}$, we have to put total weight 1 on the edges in $E^{1}$, where

$$
\begin{aligned}
E^{0}= & \left\{e_{p}^{k, 0} \mid p \in[2 n+3 ; m]^{-} \text {and } 1 \leq k \leq 3\right\} \cup \\
& \left\{e_{(0,0)}^{0}, e_{\max }^{0}\right\} \cup \\
& \left\{\left\{a_{1}, b_{1}\right\} \cup M_{1},\left\{b_{1}, c_{1}\right\} \cup M_{1},\left\{c_{1}, d_{1}\right\} \cup M_{1}\right\} \cup \\
& \left\{\left\{a_{1}^{\prime}, b_{1}^{\prime}\right\} \cup M_{1}^{\prime},\left\{b_{1}^{\prime}, c_{1}^{\prime}\right\} \cup M_{1}^{\prime},\left\{c_{1}^{\prime}, d_{1}^{\prime}\right\} \cup M_{1}^{\prime}\right\}, \\
E^{1}= & \left\{e_{p}^{k, 1} \mid p \in[2 n+3 ; m]^{-} \text {and } 1 \leq k \leq 3\right\} \cup \\
& \left\{e_{(0,0)}^{1}, e_{\max }^{1}\right\} \cup \\
& \left\{\left\{a_{2}, b_{2}\right\} \cup M_{2},\left\{b_{2}, c_{2}\right\} \cup M_{2},\left\{c_{2}, d_{2}\right\} \cup M_{2}\right\} \cup \\
& \left\{\left\{a_{2}^{\prime}, b_{2}^{\prime}\right\} \cup M_{2}^{\prime},\left\{b_{2}^{\prime}, c_{2}^{\prime}\right\} \cup M_{2}^{\prime},\left\{c_{2}^{\prime}, d_{2}^{\prime}\right\} \cup M_{2}^{\prime}\right\} .
\end{aligned}
$$

To also cover $S$ with weight 1 , we are only allowed to assign weights to the above edges. Let $e_{i}^{0}$ be an arbitrary edge in $E^{0}$ with $\gamma_{u}\left(e_{i}^{0}\right)=w_{i}>0$. Then there exists a subset $S_{i}$ of $S$ with $S_{i} \neq \emptyset$, s.t. $e_{i}^{0} \cap S=S \backslash S_{i}$. Still, we need to put weight 1 on the vertices in $S_{i}$. To do so, we can put at most weight $1-w_{i}$ on the edges in $E^{0} \backslash\left\{e_{i}^{0}\right\}$, which covers $S_{i}$ with weight at most $1-w_{i}$. Hence, the edges in $E^{1}$ have to put weight $\geq w_{i}$ on $S_{i}$. The only edge in $E^{1}$ that intersects $S_{i}$ is the complementary edge $e_{i}^{1}$ of $e_{i}^{0}$. Hence, we have to set $\gamma_{u}\left(e_{i}^{1}\right) \geq w_{i}$. In other words, we have to set $\gamma_{u}\left(e_{i}^{1}\right)=w_{i}+\epsilon_{i}$ for some $\epsilon_{i} \geq 0$. Note that $e_{i}^{0}$ was arbitrarily chosen. Hence, we can conclude for every $e_{i}^{0} \in E^{0}$ with $\gamma_{u}\left(e_{i}^{0}\right)=w_{i}>0$ and with complementary edge $e_{i}^{1} \in E^{1}$ that $\gamma_{u}\left(e_{i}^{1}\right)=w_{i}+\epsilon_{i}$ for some $\epsilon_{i} \geq 0$. Hence, 
$\sum_{e^{1} \in E^{1}} \gamma_{u}\left(e^{1}\right)=\sum_{e^{0} \in E^{0}} \gamma_{u}\left(e^{0}\right)+\sum_{i} \epsilon_{i}$. Now recall that both $\sum_{e^{0} \in E^{0}} \gamma_{u}\left(e^{0}\right)=1$ and $\sum_{e^{1} \in E^{1}} \gamma_{u}\left(e^{1}\right)=1$ hold. This is only possible if $\epsilon_{i}=0$ for every $i$. In other words, $\gamma_{u}\left(e_{i}^{0}\right)=\gamma_{u}\left(e_{i}^{1}\right)=w_{i}$ for every $e_{i}^{0} \in E^{0}$ and its complementary edge $e_{i}^{1} \in E^{1}$.

LEMMA 3.6. Let $\mathcal{F}=\left\langle T,\left(B_{u}\right)_{u \in T},\left(\gamma_{u}\right)_{u \in T}\right\rangle$ be an FHD of width $\leq 2$ of the hypergraph $H$ constructed above and let $p \in[2 n+3 ; m]^{-}$. For every node $u$ with $S \cup A_{p}^{\prime} \cup \overline{A_{p}} \cup\left\{z_{1}, z_{2}\right\} \subseteq B_{u}$, the only way to cover $S \cup A_{p}^{\prime} \cup \overline{A_{p}} \cup\left\{z_{1}, z_{2}\right\}$ by a fractional edge cover $\gamma$ of weight $\leq 2$ is by putting non-zero weight exclusively on edges $e_{p}^{k, 0}$ and $e_{p}^{k, 1}$ with $k \in\{1,2,3\}$. Moreover, $\sum_{k=1}^{3} \gamma\left(e_{p}^{k, 0}\right)=1$ and $\sum_{k=1}^{3} \gamma\left(e_{p}^{k, 1}\right)=1$ must hold.

Proof. As in the proof of Lemma 3.5, to cover $z_{1}$, we have to put weight 1 on the edges in $E^{0}$ and to cover $z_{2}$, we have to put weight 1 on the edges in $E^{1}$, where $E^{0}$ and $E^{1}$ are defined as in the proof of Lemma 3.5. Since we have width $(\mathcal{F}) \leq 2$, we have to cover $A_{p}^{\prime} \cup \overline{A_{p}} \cup S$ with the weight already put on the edges in $E^{0} \cup E^{1}$. To cover $A_{p}^{\prime}$, we have to put weight 1 on the edges in $E_{p}^{1}$, where

$$
E_{p}^{1}=\left\{e_{r}^{k, 1} \mid r \geq p\right\} \cup\left\{e_{\max }^{1}\right\} .
$$

Notice that $E_{p}^{1} \subseteq E^{1}$ and therefore $\sum_{e \in E^{1} \backslash E_{p}^{1}} \gamma_{u}(e)=0$. Similarly, to cover $\overline{A_{p}}$, we have to put weight 1 on the edges in $E_{p}^{0}$, where

$$
E_{p}^{0}=\left\{e_{s}^{k, 0} \mid s \leq p\right\} \cup\left\{e_{(0,0)}^{0}\right\} .
$$

Again, since $E_{p}^{0} \subseteq E^{0}, \sum_{e \in E^{0} \backslash E_{p}^{0}} \gamma_{u}(e)=0$. It remains to cover $S \cup\left\{z_{1}, z_{2}\right\}$. By Lemma 3.5, to cover $S, z_{1}$ and $z_{2}$, we have to put the same weight $w$ on complementary edges $e$ and $e^{\prime}$. The only complementary edges in the sets $E_{p}^{0}$ and $E_{p}^{1}$ are edges of the form $e_{p}^{k, 0}$ and $e_{p}^{k, 1}$ with $k \in\{1,2,3\}$. In total, we thus have $\sum_{k=1}^{3} e_{p}^{k, 0}=1$ and $\sum_{k=1}^{3} e_{p}^{k, 1}=1$.

Proof of the "only if"-direction. It remains to show that $\varphi$ is satisfiable if $H$ has a GHD or FHD of width $\leq 2$. Due to the inequality $f h w(H) \leq g h w(H)$, it suffices to show that $\varphi$ is satisfiable if $H$ has an FHD of width $\leq 2$. For this, let $\mathcal{F}=\left\langle T,\left(B_{u}\right)_{u \in T},\left(\gamma_{u}\right)_{u \in T}\right\rangle$ be such an FHD. Let $u_{A}, u_{B}, u_{C}$ and $u_{A}^{\prime}, u_{B}^{\prime}, u_{C}^{\prime}$ be the nodes that are guaranteed by Lemma 3.1. We state several properties of the path connecting $u_{A}$ and $u_{A}^{\prime}$, which heavily rely on Lemmas 3.5 and 3.6.

Claim A. The nodes $u_{A}^{\prime}, u_{B}^{\prime}, u_{C}^{\prime}$ (respectively, $u_{A}, u_{B}, u_{C}$ ) are not on the path from $u_{A}$ to $u_{C}$ (respectively, $u_{A}^{\prime}$ to $u_{C}^{\prime}$ ).

Proof of Claim A. We only show that none of the nodes $u_{i}^{\prime}$ with $i \in\{A, B, C\}$ is on the path from $u_{A}$ to $u_{C}$. The other property is shown analogously. Suppose to the contrary that some $u_{i}^{\prime}$ is on the path from $u_{A}$ to $u_{C}$. Since $u_{B}$ is also on the path between $u_{A}$ and $u_{C}$, we distinguish two cases:

- Case (1): $u_{i}^{\prime}$ is on the path between $u_{A}$ and $u_{B}$; then $\left\{b_{1}, b_{2}\right\} \subseteq B_{u_{i}^{\prime}}$. This contradicts the property shown in Lemma 3.1 that $u_{i}^{\prime}$ cannot cover any vertices outside $H_{0}^{\prime}$.

- Case (2): $u_{i}^{\prime}$ is on the path between $u_{B}$ and $u_{C}$; then $\left\{c_{1}, c_{2}\right\} \subseteq B_{u_{i}^{\prime}}$, which again contradicts Lemma 3.1.

Hence, the paths from $u_{A}$ to $u_{C}$ and from $u_{A}^{\prime}$ to $u_{C}^{\prime}$ are indeed disjoint.

Claim B. The following equality holds: $\operatorname{nodes}\left(A \cup A^{\prime}, \mathcal{F}\right) \cap\left\{u_{A}, u_{B}, u_{C}, u_{A}^{\prime}, u_{B}^{\prime}, u_{C}^{\prime}\right\}=\emptyset$.

Proof of Claim B. Suppose to the contrary that there is a $u_{X}$ (the proof for $u_{X}^{\prime}$ is analogous) for some $X \in\{A, B, C\}$, s.t. $u_{X} \in \operatorname{nodes}\left(A \cup A^{\prime}, \mathcal{F}\right)$; then there is some $a \in\left(A \cup A^{\prime}\right)$, s.t. $a \in$ $B_{u_{X}}$. This contradicts the property shown in Lemma 3.1 that $u_{X}$ cannot cover any vertices outside $H_{0}$. 
We are now interested in the sequence of nodes $\hat{u}_{i}$ that cover the edges $e_{(0,0)}^{0}, e_{\min }, e_{\min \oplus 1}, \ldots$, $e_{\max \ominus 1}, e_{\max }$. Before we formulate Claim $\mathrm{C}$, it is convenient to introduce the following notation: To be able to refer to the edges $e_{(0,0)}^{0}, e_{\min }, e_{\min \oplus 1}, \ldots, e_{\max } \ominus 1, e_{\max }^{1}$ in a uniform way, we use $e_{\min \ominus 1}$ as synonym of $e_{(0,0)}^{0}$ and $e_{\max }$ as synonym of $e_{\max }^{1}$. We can thus define the natural order $e_{\min \ominus 1}<e_{\min }<e_{\min \oplus 1}<\cdots<e_{\max \ominus 1}<e_{\max }$ on these edges.

Claim C. The FHD $\mathcal{F}$ has a path containing nodes $\hat{u}_{1}, \ldots, \hat{u}_{N}$ for some $N$, such that the edges $e_{\min \ominus 1}, e_{\min }, e_{\min \oplus 1}, \ldots, e_{\max \ominus 1}, e_{\max }$ are covered in this order. More formally, there is a mapping $f:\{\min \ominus 1, \ldots, \max \} \rightarrow\{1, \ldots, N\}$, s.t.

- $\hat{u}_{f(p)}$ covers $e_{p}$ and

- if $p<p^{\prime}$ then $f(p) \leq f\left(p^{\prime}\right)$.

By a path containing nodes $\hat{u}_{1}, \ldots, \hat{u}_{N}$, we mean that $\hat{u}_{1}$ and $\hat{u}_{N}$ are nodes in $\mathcal{F}$, such that the nodes $\hat{u}_{2}, \ldots, \hat{u}_{N-1}$ lie (in this order) on the path from $\hat{u}_{1}$ to $\hat{u}_{N}$. Of course, the path from $\hat{u}_{1}$ to $\hat{u}_{N}$ may also contain further nodes, but we are not interested in whether they cover any of the edges $e_{p}$.

Proof of Claim C. Suppose to the contrary that no such path exists. Let $p \geq \min$ be the maximal value such that there is a path containing nodes $\hat{u}_{1}, \hat{u}_{2}, \ldots, \hat{u}_{\ell}$, which cover $e_{\min \ominus 1}, \ldots, e_{p}$ in this order. Clearly, there exists a node $\hat{u}$ that covers $e_{p \oplus 1}=A_{p \oplus 1}^{\prime} \cup \overline{A_{p \oplus 1}}$. We distinguish four cases:

- Case (1): $\hat{u}_{1}$ is on the path from $\hat{u}$ to all other nodes $\hat{u}_{i}$, with $1<i \leq \ell$. By the connectedness condition, $\hat{u}_{1}$ covers $A_{p}^{\prime}$. Hence, in total $\hat{u}_{1}$ covers $A_{p}^{\prime} \cup A$ with $A_{p}^{\prime}=\left\{a_{\min }^{\prime}, \ldots, a_{p}^{\prime}\right\}$ and $A=\left\{a_{\min }, \ldots, a_{\max }\right\}$. Then $\hat{u}_{1}$ covers all edges $e_{\min \ominus 1}, \ldots, e_{p}$. Therefore, the path containing nodes $\hat{u}_{1}$ and $\hat{u}$ covers $e_{\min } \ominus 1, \ldots, e_{p \oplus 1}$ in this order, which contradicts the maximality of $p$.

- Case (2): $\hat{u}=\hat{u}_{1}$, hence, $\hat{u}_{1}$ covers $A_{p \oplus 1}^{\prime} \cup A$ with $A_{p \oplus 1}^{\prime}=\left\{a_{\min }^{\prime}, \ldots, a_{p \oplus 1}^{\prime}\right\}$ and $A=\left\{a_{\min }, \ldots\right.$, $\left.a_{\max }\right\}$. Then, $\hat{u}_{1}$ covers all $e_{\min } \ominus 1, \ldots, e_{p \oplus 1}$, which contradicts the maximality of $p$.

- Case (3): $\hat{u}$ is on the path from $\hat{u}_{1}$ to $\hat{u}_{\ell}$ and $\hat{u} \neq \hat{u}_{1}$. Hence, $\hat{u}$ is between two nodes $\hat{u}_{i}$ and $\hat{u}_{i+1}$ for some $1 \leq i<\ell$ or $\hat{u}=\hat{u}_{i+1}$ for some $1 \leq i<\ell-1$. The following arguments hold for both cases: Now, there is some $q \leq p$, such that $e_{q}$ is covered by $\hat{u}_{i+1}$ and $e_{q \ominus 1}$ is covered by $\hat{u}_{i}$. Therefore, $\hat{u}$ covers $\overline{A_{q}}$ either by the connectedness condition (if $\hat{u}$ is between $\hat{u}_{i}$ and $\left.\hat{u}_{i+1}\right)$ or simply because $\hat{u}=\hat{u}_{i+1}$. Hence, in total, $\hat{u}$ covers $A_{p \oplus 1}^{\prime} \cup \overline{A_{q}}$ with $A_{p \oplus 1}^{\prime}=\left\{a_{\min }^{\prime}, \ldots, a_{p \oplus 1}^{\prime}\right\}$ and $\overline{A_{q}}=\left\{a_{q}, a_{q \oplus 1}, \ldots, a_{p}, a_{p \oplus 1}, \ldots a_{\max }\right\}$. Then, $\hat{u}$ covers all edges $e_{q}, e_{q \oplus 1}, \ldots, e_{p \oplus 1}$. Therefore, the path containing nodes $\hat{u}_{1}, \ldots, \hat{u}_{i}, \hat{u}$ covers $e_{\min \ominus 1}, \ldots, e_{p \oplus 1}$ in this order, which contradicts the maximality of $p$.

- Case (4): There is a $u^{*}$ on the path from $\hat{u}_{1}$ to $\hat{u}_{\ell}$, such that the paths from $\hat{u}_{1}$ to $\hat{u}$ and from $\hat{u}$ to $\hat{u}_{\ell}$ go through $u^{*}$ and, moreover, $u^{*} \neq \hat{u}_{1}$. Then, $u^{*}$ is either between $\hat{u}_{i}$ and $\hat{u}_{i+1}$ for some $1 \leq i<\ell$ or $u^{*}=\hat{u}_{i+1}$ for some $1 \leq i<\ell-1$. The following arguments hold for both cases: There is some $q \leq p$, such that $e_{q}$ is covered by $\hat{u}_{i+1}$ and $e_{q \ominus 1}$ is covered by $\hat{u}_{i}$. By the connectedness condition, $u^{*}$ covers

- $A_{p}^{\prime}=\left\{a_{\min }^{\prime}, \ldots, a_{p}^{\prime}\right\}$, since $u^{*}$ is on the path from $\hat{u}$ to $\hat{u}_{\ell}$, and

- $\bar{A}_{q}=\left\{a_{q}, \ldots, a_{p}, a_{p \oplus 1}, \ldots a_{\max }\right\}$, since $u^{*}$ is on the path from $\hat{u}_{1}$ to $\hat{u}_{i+1}$ or $u^{*}=\hat{u}_{i+1}$.

Then $u^{*}$ covers all edges $e_{q}, e_{q \oplus 1}, \ldots, e_{p}$. Therefore, the path containing the nodes $\hat{u}_{1}, \ldots, \hat{u}_{i}$, $u^{*}, \hat{u}$ covers $e_{\min \ominus 1}, \ldots, e_{p \oplus 1}$ in this order, which contradicts the maximality of $p$.

So far, we have shown that there are three disjoint paths from $u_{A}$ to $u_{C}$, from $u_{A}^{\prime}$ to $u_{C}^{\prime}$ and from $\hat{u}_{1}$ to $\hat{u}_{N}$, respectively. It is easy to see that $u_{A}$ is closer to the path $\hat{u}_{1}, \ldots, \hat{u}_{N}$ than $u_{B}$ and $u_{C}$, since otherwise $u_{B}$ and $u_{C}$ would have to cover $a_{1}$ as well, which is impossible by Lemma 3.1. The same also holds for $u_{A}^{\prime}$. In the next claims, we will argue that the path from $u_{A}$ to $u_{A}^{\prime}$ goes through some 


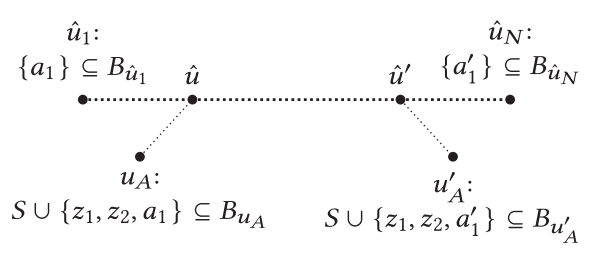

(a)

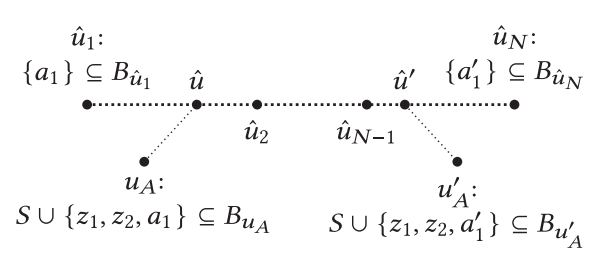

(b)

Fig. 4. Arrangement of the nodes $\hat{u}_{1}, \hat{u}, \hat{u}^{\prime}$, and $\hat{u}_{N}$ from Claim E (a) and Claim G (b).

node $\hat{u}$ of the path from $\hat{u}_{1}$ to $\hat{u}_{N}$. We write $\pi\left(\hat{u}_{1}, \hat{u}_{N}\right)$ as a short-hand notation for the path from $\hat{u}_{1}$ to $\hat{u}_{N}$. Next, we state some important properties of $\pi\left(\hat{u}_{1}, \hat{u}_{N}\right)$ and the path from $u_{A}$ to $u_{A}^{\prime}$.

CLAIM D. In the FHD $\mathcal{F}$ of $H$ of width $\leq 2$, the path from $u_{A}$ to $u_{A}^{\prime}$ has non-empty intersection with $\pi\left(\hat{u}_{1}, \hat{u}_{N}\right)$.

Proof of Claim D. Suppose to the contrary that the path from $u_{A}$ to $u_{A}^{\prime}$ is disjoint from $\pi\left(\hat{u}_{1}, \hat{u}_{N}\right)$. We distinguish three cases:

- Case (1): $u_{A}$ is on the path from $u_{A}^{\prime}$ to (some node in) $\pi\left(\hat{u}_{1}, \hat{u}_{N}\right)$. Then, by the connectedness condition, $u_{A}$ must contain $a_{1}^{\prime}$, which contradicts Lemma 3.1.

- Case (2): $u_{A}^{\prime}$ is on the path from $u_{A}$ to $\pi\left(\hat{u}_{1}, \hat{u}_{N}\right)$. Analogously to Case (1), we get a contradiction by the fact that then $u_{A}^{\prime}$ must contain $a_{1}$.

- Case (3): There is a node $u^{*}$ on the path from $u_{A}$ to $u_{A}^{\prime}$, which is closest to $\pi\left(\hat{u}_{1}, \hat{u}_{N}\right)$, i.e., $u^{*}$ lies on the path from $u_{A}$ to $u_{A}^{\prime}$ and both paths, the one connecting $u_{A}$ with $\pi\left(\hat{u}_{1}, \hat{u}_{N}\right)$ and the one connecting $u_{A}^{\prime}$ with $\pi\left(\hat{u}_{1}, \hat{u}_{N}\right)$, go through $u^{*}$. Hence, by the connectedness condition, the bag of $u^{*}$ contains $S \cup\left\{z_{1}, z_{2}, a_{1}, a_{1}^{\prime}\right\}$. By Lemma 3.5, to cover $S \cup\left\{z_{1}, z_{2}\right\}$ with weight $\leq 2$, we are only allowed to put non-zero weight on pairs of complementary edges. However, then it is impossible to achieve also weight $\geq 1$ on $a_{1}$ and $a_{1}^{\prime}$ at the same time.

CLAIM E. In the FHD $\mathcal{F}$ of $H$ of width $\leq 2$ there are two distinct nodes $\hat{u}$ and $\hat{u}^{\prime}$ in the intersection of the path from $u_{A}$ to $u_{A}^{\prime}$ with $\pi\left(\hat{u}_{1}, \hat{u}_{N}\right)$, s.t. $\hat{u}$ is the node in $\pi\left(\hat{u}_{1}, \hat{u}_{N}\right)$ closest to $u_{A}$ and $\hat{u}^{\prime}$ is the node in $\pi\left(\hat{u}_{1}, \hat{u}_{N}\right)$ closest to $u_{A}^{\prime}$. Then, on the path $\pi\left(\hat{u}_{1}, \hat{u}_{N}\right)$, $\hat{u}$ comes before $\hat{u}^{\prime}$. See Figure 4 (a) for a graphical illustration of the arrangement of the nodes $\hat{u}_{1}, \hat{u}, \hat{u}^{\prime}$, and $\hat{u}_{N}$ on the path $\pi\left(\hat{u}_{1}, \hat{u}_{N}\right)$.

Proof of Claim E. First, we show that $\hat{u}$ and $\hat{u}^{\prime}$ are indeed distinct. Suppose towards a contradiction that they are not, i.e., $\hat{u}=\hat{u}^{\prime}$. Then, by connectedness, $\hat{u}$ has to cover $S \cup\left\{z_{1}, z_{2}\right\}$, because $S \cup\left\{z_{1}, z_{2}\right\}$ is contained in $B_{u_{A}}$ and in $B_{u_{A}^{\prime}}$. Moreover, again by connectedness, $\hat{u}$ also has to cover $\left\{a_{1}, a_{1}^{\prime}\right\}$, because $a_{1}$ is contained in $B_{\hat{u}_{1}}$ and in $B_{u_{A}}$ and $a_{1}^{\prime}$ is contained in $B_{\hat{u}_{N}}$ and in $B_{u_{A}^{\prime}}$. As in Case (3) in the proof of Claim D, this is impossible by Lemma 3.5. Hence, $\hat{u}$ and $\hat{u}^{\prime}$ are distinct.

Second, we show that, on the path from $\hat{u}_{1}$ to $\hat{u}_{N}$, the node $\hat{u}$ comes before $\hat{u}^{\prime}$. Suppose to the contrary that $\hat{u}^{\prime}$ comes before $\hat{u}$. Then, by the connectedness condition, $\hat{u}$ covers the following (sets of) vertices:

- $a_{1}^{\prime}$, since we are assuming that $\hat{u}^{\prime}$ comes before $\hat{u}$, i.e., $\hat{u}$ is on the path from $\hat{u}_{N}$ to $u_{A}^{\prime}$;

- $a_{1}$, since $\hat{u}$ is on the path from $\hat{u}_{1}$ to $u_{A}$;

- $S \cup\left\{z_{1}, z_{2}\right\}$, since $\hat{u}$ is on the path from $u_{A}$ to $u_{A}^{\prime}$.

In total, $\hat{u}$ has to cover all vertices in $S \cup\left\{z_{1}, z_{2}, a_{1}, a_{1}^{\prime}\right\}$. Again, by Lemma 3.5, this is impossible with weight $\leq 2$.

CLAIM F. In the FHD F of $H$ of width $\leq 2$ the path $\pi\left(\hat{u}_{1}, \hat{u}_{N}\right)$ has at least three nodes $\hat{u}_{i}$, i.e., $N \geq 3$. 
Proof of Claim F. First, it is easy to verify that $N \geq 2$ must hold. Otherwise, a single node would have to cover $\left\{e_{\min \ominus 1}, e_{\min }, e_{\min \oplus 1}, \ldots, e_{\max \ominus 1}, e_{\max }\right\}$ and, hence, in particular, $S \cup\left\{z_{1}, z_{2}, a_{1}, a_{1}^{\prime}\right\}$, which is impossible, as we have already seen in Case (3) of the proof of Claim D.

It remains to prove $N \geq 3$. Suppose to the contrary that $N=2$. By the problem reduction, hypergraph $H$ has distinct edges $e_{\min \ominus 1}, e_{\min }$ and $e_{\max }$. Hence, $\hat{u}_{1}$ covers at least $e_{\min } \ominus 1$ and $\hat{u}_{2}$ covers at least $e_{\max }$. Recall from Claim $\mathrm{E}$ the nodes $\hat{u}$ and $\hat{u}^{\prime}$, which constitute the endpoints of the intersection of the path from $u_{A}$ to $u_{A}^{\prime}$ with the path $\pi\left(\hat{u}_{1}, \hat{u}_{N}\right)$ (cf. Figure $4(\mathrm{a})$ ). Here, we are assuming $N=2$. We now show that, by the connectedness condition of FHDs, the nodes $\hat{u}$ and $\hat{u}^{\prime}$ must cover certain vertices, which will lead to a contradiction by Lemma 3.5.

- vertices covered by $\hat{u}$ : node $\hat{u}$ is on the path between $u_{A}$ and $u_{A}^{\prime}$. Hence, it covers $S \cup\left\{z_{1}, z_{2}\right\}$. Moreover, $\hat{u}$ is on the path between $\hat{u}_{1}$ and $u_{A}$ (or even coincides with $\hat{u}_{1}$ ). Hence, it also covers $a_{1}$. In total, $\hat{u}$ covers at least $S \cup\left\{z_{1}, z_{2}, a_{1}\right\}$.

- vertices covered by $\hat{u}^{\prime}$ : node $\hat{u}^{\prime}$ is on the path between $u_{A}$ and $u_{A}^{\prime}$. Hence, it covers $S \cup\left\{z_{1}, z_{2}\right\}$. Moreover, $\hat{u}^{\prime}$ is on the path between $\hat{u}_{2}$ and $u_{A}^{\prime}$ (or even coincides with $\hat{u}_{2}$ ). Hence, it also covers $a_{1}^{\prime}$. In total, $\hat{u}^{\prime}$ covers at least $S \cup\left\{z_{1}, z_{2}, a_{1}^{\prime}\right\}$.

One of the nodes $\hat{u}_{1}$ or $\hat{u}_{2}$ must also cover the edge $e_{\text {min }}$. We inspect these two cases separately:

- Case (1): suppose that the edge $e_{\min }$ is covered by $\hat{u}_{1}$. Then, $\hat{u}_{1}$ covers vertex $a_{\min }^{\prime}$, which is also covered by $\hat{u}_{2}$. Hence, also $\hat{u}$ covers $a_{\min }^{\prime}$. In total, $\hat{u}$ covers $S \cup\left\{z_{1}, z_{2}, a_{1}, a_{\min }^{\prime}\right\}$. However, by Lemma 3.5, we know that, to cover $S \cup\left\{z_{1}, z_{2}\right\}$ with weight $\leq 2$, we are only allowed to put non-zero weight on pairs of complementary edges. Hence, it is impossible to achieve also weight $\geq 1$ on $a_{1}$ and on $a_{\min }^{\prime}$ at the same time.

- Case (2): suppose that the edge $e_{\min }$ is covered by $\hat{u}_{2}$. Then, $\hat{u}_{2}$ covers vertex $a_{\min }$ (actually, it even covers all of $A$ ), which is also covered by $\hat{u}_{1}$. Hence, also $\hat{u}^{\prime}$ covers $a_{\min }$. In total, $\hat{u}^{\prime}$ covers $S \cup\left\{z_{1}, z_{2}, a_{1}^{\prime}, a_{\min }\right\}$. Again, this is impossible by Lemma 3.5.

Hence, the path $\pi\left(\hat{u}_{1}, \hat{u}_{N}\right)$ indeed has at least three nodes $\hat{u}_{i}$.

CLAIM G. In the FHD $\mathcal{F}$ of $H$ of width $\leq 2$ all the nodes $\hat{u}_{2}, \ldots, \hat{u}_{N-1}$ are on the path from $u_{A}$ to $u_{A}^{\prime}$. For the nodes $\hat{u}$ and $\hat{u}^{\prime}$ from Claim $E$, this means that the nodes $\hat{u}_{1}, \hat{u}, \hat{u}_{2}, \hat{u}_{N-1}, \hat{u}^{\prime}, \hat{u}_{N}$ are arranged in precisely this order on the path $\pi\left(\hat{u}_{1}, \hat{u}_{N}\right)$ from $\hat{u}_{1}$ to $\hat{u}_{N}$ (cf. Figure $\left.4(b)\right)$. The node $\hat{u}$ may possibly coincide with $\hat{u}_{1}$ and $\hat{u}^{\prime}$ may possibly coincide with $\hat{u}_{N}$.

Proof of Claim G. We have to prove that $\hat{u}$ lies between $\hat{u}_{1}$ and $\hat{u}_{2}$ (not including $\hat{u}_{2}$ ) and $\hat{u}^{\prime}$ lies between $\hat{u}_{N-1}$ and $\hat{u}_{N}$ (not including $\hat{u}_{N-1}$ ). For the first property, suppose to the contrary that $\hat{u}$ does not lie between $\hat{u}_{1}$ and $\hat{u}_{2}$ or $\hat{u}=\hat{u}_{2}$. This means that there exists $i \in\{2, \ldots, N-1\}$ such that $\hat{u}$ lies between $\hat{u}_{i}$ and $\hat{u}_{i+1}$, including the case that $\hat{u}$ coincides with $\hat{u}_{i}$. Note that, by Claim E, $\hat{u}$ cannot coincide with $\hat{u}_{N}$, since there is yet another node $\hat{u}^{\prime}$ between $\hat{u}$ and $\hat{u}_{N}$.

By definition of $\hat{u}_{i}$ and $\hat{u}_{i+1}$, there is a $p \in[2 n+3 ; m]$, such that both $\hat{u}_{i}$ and $\hat{u}_{i+1}$ cover $a_{p}^{\prime}$. Then, by the connectedness condition, $\hat{u}$ covers the following (sets of) vertices:

- $a_{p}^{\prime}$, since $\hat{u}$ is on the path from $\hat{u}_{i}$ to $\hat{u}_{i+1}$ (or $\hat{u}$ coincides with $\hat{u}_{i}$ ),

- $a_{1}$, since $\hat{u}$ is on the path from $\hat{u}_{1}$ to $u_{A}$,

- $S \cup\left\{z_{1}, z_{2}\right\}$, since $\hat{u}$ is on the path from $u_{A}$ to $u_{A}^{\prime}$.

However, by Lemma 3.5, we know that, to cover $S \cup\left\{z_{1}, z_{2}\right\}$ with weight $\leq 2$, we are only allowed to put non-zero weight on pairs of complementary edges. Hence, it is impossible to achieve also weight $\geq 1$ on $a_{p}^{\prime}$ and $a_{1}$ at the same time.

It remains to show that $\hat{u}^{\prime}$ lies between $\hat{u}_{N-1}$ and $\hat{u}_{N}$ (not including $\hat{u}_{N-1}$ ). Suppose to the contrary that it does not. Then, analogously to the above considerations for $\hat{u}$, it can be shown that 
there exists some $p \in[2 n+3 ; m]$, such that $\hat{u}^{\prime}$ covers the vertices $S \cup\left\{z_{1}, z_{2}, a_{p}, a_{1}^{\prime}\right\}$. Again, this is impossible by Lemma 3.5.

By Claim C, the decomposition $\mathcal{F}$ contains a path $\hat{u}_{1} \cdots \hat{u}_{N}$ that covers the edges $e_{\min } \ominus 1, e_{\min }$, $e_{\min \oplus 1}, \ldots, e_{\max } \ominus 1, e_{\max }$ in this order. We next strengthen this property by showing that every node $\hat{u}_{i}$ covers exactly one edge $e_{p}$.

Claim H. Each of the nodes $\hat{u}_{1}, \ldots, \hat{u}_{N}$ covers exactly one of the edges $e_{\min } \ominus 1, e_{\min }, e_{\min } \oplus 1, \ldots$, $e_{\max \ominus 1}, e_{\max }$.

Proof of Claim H. We prove this property for the "outer nodes" $\hat{u}_{1}, \hat{u}_{N}$ and for the "inner nodes" $\hat{u}_{2} \cdots \hat{u}_{N-1}$ separately. We start with the "outer nodes." The proof for $\hat{u}_{1}$ and $\hat{u}_{N}$ is symmetric. We thus only work out the details for $\hat{u}_{1}$. Suppose to the contrary that $\hat{u}_{1}$ not only covers $e_{\min } \ominus 1$ but also $e_{\min }$. We distinguish two cases according to the position of node $\hat{u}$ in Figure 4(b):

- Case (1): $\hat{u}=\hat{u}_{1}$. Then, $\hat{u}_{1}$ has to cover the following (sets of) vertices:

$-S \cup\left\{z_{1}, z_{2}\right\}$, since $\hat{u}$ is on the path from $u_{A}$ to $u_{A}^{\prime}$ and we are assuming $\hat{u}=\hat{u}_{1}$.

- $a_{1}$, since $\hat{u}_{1}$ covers $e_{\min \ominus 1}$,

- $a_{\min }^{\prime}$, since we are assuming that $\hat{u}_{1}$ also covers $e_{\min }$.

By applying Lemma 3.5, we may conclude that the set $S \cup\left\{z_{1}, z_{2}, a_{1}, a_{\min }^{\prime}\right\}$ cannot be covered by a fractional edge cover of weight $\leq 2$.

- Case (2): $\hat{u} \neq \hat{u}_{1}$. Then $\hat{u}$ is on the path from $\hat{u}_{1}$ to $\hat{u}_{2}$. Hence, $\hat{u}$ has to cover the following (sets of) vertices:

$-S \cup\left\{z_{1}, z_{2}\right\}$, since $\hat{u}$ is on the path from $u_{A}$ to $u_{A}^{\prime}$,

$-a_{1}$, since $\hat{u}$ is on the path from $u_{A}$ to $\hat{u}_{1}$,

- $a_{\text {min }}^{\prime}$, since $\hat{u}$ is on the path from $\hat{u}_{1}$ to $\hat{u}_{2}$.

As in Case (1) above, $S \cup\left\{z_{1}, z_{2}, a_{1}, a_{\min }^{\prime}\right\}$ cannot be covered by a fractional edge cover of weight $\leq 2$ due to Lemma 3.5 .

It remains to consider the "inner" nodes $\hat{u}_{i}$ with $2 \leq i \leq N-1$. Each such $\hat{u}_{i}$ has to cover $S \cup\left\{z_{1}, z_{2}\right\}$, since all these nodes are on the path from $u_{A}$ to $u_{A}^{\prime}$ by Claim G. Now suppose that $\hat{u}_{i}$ covers $e_{p}=A_{p}^{\prime} \cup \overline{A_{p}}$ for some $p \in\left\{e_{\min }, \ldots, e_{\max } \ominus 1\right\}$. By Lemma 3.6, covering all of the vertices $A_{p}^{\prime} \cup \overline{A_{p}} \cup S \cup\left\{z_{1}, z_{2}\right\}$ by a fractional edge cover of weight $\leq 2$ requires that we put total weight 1 on the edges $e_{p}^{k, 0}$ and total weight 1 on the edges $e_{p}^{k, 1}$ with $k \in\{1,2,3\}$. However, then it is impossible to cover also $e_{p^{\prime}}$ for some $p^{\prime}$ with $p^{\prime} \neq p$. This concludes the proof of Claim F.

We can now associate with each $\hat{u}_{i}$ for $1 \leq i \leq N$ the corresponding edge $e_{p}$ and write $u_{p}$ to denote the node that covers the edge $e_{p}$. By Claim $G$, we know that all of the nodes $u_{\min } \ldots, u_{\max } \ominus 1$ are on the path from $u_{A}$ to $u_{A}^{\prime}$. Hence, by the connectedness condition, all these nodes cover $S \cup$ $\left\{z_{1}, z_{2}\right\}$.

We are now ready to construct a satisfying truth assignment $\sigma$ of $\varphi$. For each $i \leq 2 n+3$, let $X_{i}$ be the set $B_{u_{(i, 1)}} \cap\left(Y \cup Y^{\prime}\right)$. As $Y \subseteq B_{u_{A}}$ and $Y^{\prime} \subseteq B_{u_{A}^{\prime}}$, the sequence $X_{1} \cap Y, \ldots, X_{2 n+3} \cap Y$ is non-increasing and the sequence $X_{1} \cap Y^{\prime}, \ldots, X_{2 n+3} \cap Y^{\prime}$ is non-decreasing. Furthermore, as all edges $e_{y_{i}}=\left\{y_{i}, y_{i}^{\prime}\right\}$ must be covered by some node in $\mathcal{F}$, we conclude that for each $i$ and $j, y_{j} \in X_{i}$ or $y_{j}^{\prime} \in X_{i}$. Then, there is some $s \leq 2 n+2$ such that $X_{s}=X_{s+1}$. Furthermore, all nodes between $u_{(s, 1)}$ and $u_{(s+1,1)}$ cover $X_{s}$. We derive a truth assignment for $x_{1}, \ldots, x_{n}$ from $X_{s}$ as follows: For each $\ell \leq n$, we set $\sigma\left(x_{\ell}\right)=1$ if $y_{\ell} \in X_{s}$ and otherwise $\sigma\left(x_{\ell}\right)=0$. Note that in the latter case $y_{\ell}^{\prime} \in X_{s}$.

CLAIM I. The constructed truth assignment $\sigma$ is a model of $\varphi$. 
Proof of Claim I. We have to show that every clause $c_{j}=L_{j}^{1} \vee L_{j}^{2} \vee L_{j}^{3}$ of $\varphi$ is true in $\sigma$. Choose an arbitrary $j \in\{1, \ldots, m\}$. We have to show that there exists a literal in $c_{j}$ that is true in $\sigma$. To this end, we inspect the node $u_{(s, j)}$, which, by construction, lies between $u_{(s, 1)}$ and $u_{(s+1,1)}$. Let $p=(s, j)$. Then, we have $A_{p}^{\prime} \cup \overline{A_{p}} \cup S \cup\left\{z_{1}, z_{2}\right\} \subseteq B_{u_{p}}$. Moreover, by the definition of $X_{s}$, we also have $X_{s} \subseteq B_{u_{p}}$. By Lemma 3.6, the only way to cover $B_{u_{p}}$ with weight $\leq 2$ is by using exclusively the edges $e_{p}^{k, 0}$ and $e_{p}^{k, 1}$ with $k \in\{1,2,3\}$. More specifically, we have $\sum_{k=1}^{3} \gamma_{u_{p}}\left(e_{p}^{k, 0}\right)=1$ and $\sum_{k=1}^{3} \gamma_{u_{p}}\left(e_{p}^{k, 1}\right)=1$. Therefore, $\gamma_{u_{p}}\left(e_{p}^{k, 0}\right)>0$ for some $k$. We distinguish two cases depending on the form of literal $L_{j}^{k}$ :

- Case (1): First, suppose $L_{j}^{k}=x_{\ell}$. By Lemma 3.5, complementary edges must have equal weight. Hence, from $\gamma_{u_{p}}\left(e_{p}^{k, 0}\right)>0$ it follows that also $\gamma_{u_{p}}\left(e_{p}^{k, 1}\right)>0$ holds. Thus, the weight on $y_{\ell}^{\prime}$ is less than 1 , which means that $y_{\ell}^{\prime} \notin B\left(\gamma_{u_{p}}\right)$ and consequently $y_{\ell}^{\prime} \notin X_{s}$. Since this implies that $y_{\ell} \in X_{s}$, we indeed have that $\sigma\left(x_{\ell}\right)=1$.

- Case (2): Conversely, suppose $L_{j}^{k}=\neg x_{\ell}$. Since $\gamma_{u_{p}}\left(e_{p}^{k, 0}\right)>0$, the weight on $y_{\ell}$ is less than 1 , which means that $y_{\ell} \notin B\left(\gamma_{u_{p}}\right)$ and consequently $y_{\ell} \notin X_{s}$. Hence, we have $\sigma\left(x_{\ell}\right)=0$.

In either case, literal $L_{p}^{k}$ is satisfied by $\sigma$ and therefore, the $j$ th clause $c_{j}$ is satisfied by $\sigma$. Since $j$ was arbitrarily chosen, $\sigma$ indeed satisfies $\varphi$.

Claim I completes the proof of Theorem 3.2.

We conclude this section by mentioning that the above reduction is easily extended to $k+\ell$ for arbitrary $\ell \geq 1$ : For integer values $\ell$, simply add a clique of $2 \ell$ fresh vertices $v_{1}, \ldots, v_{2 \ell}$ to $H$ and connect each $v_{i}$ with each "old" vertex in $H$. Now assume a rational value $\ell \geq 1$, i.e., $\ell=r / q$ for natural numbers $r, q$ with $r>q>0$. To achieve a rational bound $k+r / q$, we add $r$ fresh vertices and add hyperedges $\left\{v_{i}, v_{i \oplus 1}, \ldots, v_{i \oplus(q-1)}\right\}$ with $i \in\{1, \ldots, r\}$ to $H$, where $a \oplus b$ denotes $a+b$ modulo $r$. Again, we connect each $v_{i}$ with each "old" vertex in $H$. With this construction, we can give NP-hardness proofs for any (fractional) $k \geq 3$. For all fractional values $k<3$ (except for $k=2$ ) different gadgets and ideas might be needed to prove NP-hardness of Снеск(FHD, $k$ ), which we leave for future work.

\section{A FRAMEWORK FOR EFFICIENT COMPUTATION OF DECOMPOSITIONS}

Before we move on to the easy cases for $\operatorname{CHEck}(\mathrm{GHD}, k)$ and $\mathrm{CHECK}(\mathrm{FHD}, k)$, we will introduce a framework for a uniform presentation of the tractability proofs in the following sections. Conceptually, we can split the task of checking whether a decomposition of certain width exists into two parts: (1) deciding which sets of vertices are acceptable as bags (the candidate bags) and (2) deciding if there is a tree decomposition made up of only acceptable bags. In this section, we focus on the second part and show that this task is indeed tractable as long as the decompositions satisfy a certain normal form. This will allow us to show the СНЕск problem tractable for settings where we can compute an appropriate set of candidate bags in polynomial time.

To emphasize the generality of the approach, we will focus on tree decompositions in this section. Recall that a generalized hypertree decomposition of width at most $k$ is simply a tree decomposition where every bag has an integral edge cover with weight at most $k$. The same is true for fractional hypertree decompositions and fractional edge covers. Hence, the CHEck(GHD, k) problem can be solved by computing appropriate sets $\mathrm{S}$ of candidate bags that can be covered by $k$ edges and then deciding whether there exists a TD using only bags from S. If such a TD exists, then it is a witness for the existence of a GHD of width at most $k$. Of course, the same strategy also works for $\mathrm{CHECK}(\mathrm{FHD}, \mathrm{k})$. 
First, we will formally define the task we are interested in as the candidate tree decomposition problem. We show that the problem is NP-complete even for acyclic graphs. Following that, we show that the problem becomes tractable if we limit our search to finding TDs that adhere to a certain normal form that is sufficient for our purposes.

Definition 4.1. Let $H$ be a hypergraph and $\mathcal{T}=\left\langle T,\left(B_{u}\right)_{u \in T}\right\rangle$ be a tree decomposition of $H$. Let the candidate bags $\mathrm{S}$ be a family of subsets of $V(H)$. If for each $u \in T$ there exists an $S \in \mathrm{S}$ such that $B_{u}=S$, then we call $\mathcal{T}$ a candidate tree decomposition of $\mathrm{S}$. We denote by CTD(S) the set of all candidate tree decompositions of $H$.

Theorem 4.2. Let $H$ be a hypergraph and $\mathrm{S} \subseteq 2^{V(H)}$. It is NP-complete to decide whether $\mathrm{CTD}(\mathrm{S}) \neq$ $\emptyset$. The problem remains NP-complete even if we restrict the choice of $\mathrm{H}$ to acyclic graphs.

Proof. The problem is clearly in NP. We show NP-hardness by reduction from the exact cover problem: Let $U=\left\{u_{1}, \ldots, u_{n}\right\}$ be the universe and let $X_{1}, \ldots, X_{m}$ be subsets of $U$. The exact cover problem asks for a cover of $U$ by elements of $\left\{X_{1}, \ldots, X_{m}\right\}$ such that the sets in the cover are pairwise disjoint.

We define an acyclic graph $G$ as follows: $G$ is a tree with vertices $v, v_{1}, \ldots v_{n}, u_{1}, \ldots, u_{n}$. The edges of $G$ are $\left\{v, v_{i}\right\}$ and $\left\{v_{i}, u_{i}\right\}$ for each $1 \leq i \leq n$. Let $S=\left\{S, S_{1}, \ldots, S_{m}\right\}$ where $S=\left\{v, v_{1}, \ldots v_{n}\right\}$ and each $S_{i}=X_{i} \cup\left\{v_{j} \mid u_{j} \in X_{i}\right\}$, i.e., by taking $X_{i}$ and adding $v_{j}$ for each $u_{j}$ contained in $X_{i}$.

We claim that then $\mathrm{CTD}(\mathrm{S}) \neq \emptyset$ iff there is an exact cover of $U$ by $X_{1}, \ldots, X_{m}$. One direction is easy. Let $\mathrm{X} \subseteq\left\{X_{1}, \ldots, X_{m}\right\}$ be an exact cover of $U$. Denote the elements of $\mathrm{X}$ by $X_{1}^{\prime}, \ldots, X_{q}^{\prime}$. For $1 \leq i \leq q$, let $S_{i}^{\prime}$ be obtained from $X_{i}^{\prime}$ by adding $v_{i}$ for each $u_{i} \in X_{i}^{\prime}$. Clearly $S_{i}^{\prime} \in\left\{S_{1}, \ldots, S_{m}\right\}$ and there exists a candidate tree decomposition $\left\langle T,\left(B_{u}\right)_{u \in T}\right\rangle$ of $G$ where $V(T)=\left\{t, t_{1}, \ldots, t_{q}\right\}$, $E(T)=\left\{\left\{t, t_{1}\right\}, \ldots,\left\{t, t_{q}\right\}\right\}, B_{t}=S$ and for each $1 \leq i \leq q, B_{t_{i}}=S_{i}^{\prime}$.

The other direction is more complicated. Let $\left\langle T,\left(B_{u}\right)_{u \in T}\right\rangle$ be a smallest (w.r.t. the number of nodes of $T$ ) candidate TD of $G$. In particular, this means that the bags of all the nodes are distinct. The proof proceeds in several steps:

(1) There is $t \in V(T)$ such that $B_{t}=S$. Indeed, otherwise, $v$ is not covered.

(2) For each $1 \leq i \leq n$, there is $t^{\prime} \in V(T)$ such that $u_{i} \in B_{t^{\prime}}$ and $t$ is adjacent to $t^{\prime}$. Indeed, assume the opposite and let $t^{\prime} \in V(T)$ be a node with $u_{i} \in B_{t^{\prime}}$ such that $t$ is not adjacent to $t^{\prime}$.

Since we assume a candidate TD, we have $B_{t^{\prime}}=S_{j}$ for some $j$ and hence $v_{i} \in B_{t^{\prime}}$. Now consider the path between $t$ and $t^{\prime}$ and let $s$ be the node next to $t$ on this path. By our minimality assumption, we know that $B_{s} \neq B_{t}$ and thus $B_{s}=S_{k}$ with $k \neq j$. Since we assume the claim to be false, we have $u_{i} \notin S_{k}$ and hence $v_{i} \notin S_{k}$. As $v_{i} \in B_{t^{\prime}}$ and $v_{i} \in B_{t}$, the connectedness condition of the tree decomposition is violated.

(3) Let $t_{1}, \ldots, t_{q}$ be the neighbors of $t$ in $T$. We claim that $T$ has no other nodes. Indeed, all the vertices of $G$ are covered by the bags of $t, t_{1}, \ldots t_{q}$ by the previous two items. Each edge $\left\{v, v_{i}\right\}$ is contained in $B_{t}$. Also, each edge $\left\{u_{i}, v_{i}\right\}$ is covered by some $B_{t_{j}}$ containing $u_{i}$ (existing by the previous item). It follows that $T\left[\left\{t, t_{1}, \ldots, t_{q}\right\}\right]$ together with the corresponding bags form a tree decomposition of $G$. By the minimality assumption, $T$ does not have other nodes.

(4) We claim that for any $1 \leq i \neq j \leq q, B_{t_{i}} \cap B_{t_{j}} \neq \emptyset$. Indeed, otherwise, there is $u_{k} \in$ $B_{t_{i}} \cap B_{t_{j}}$. However, $u_{k} \notin B_{t}$ in contradiction to the connectedness condition. It follows that $B_{t_{1}} \cap U, \ldots, B_{t_{q}} \cap U$ are disjoint elements of $\left\{X_{1}, \ldots, X_{m}\right\}$ covering all of $U$ as required.

To obtain a tractable version of the problem, we will introduce a generalization of the normal form that was used in the tractability proof for HDs in Reference [31]. 
Definition 4.3. A tree decomposition $\left\langle T,\left(B_{u}\right)_{u \in T}\right\rangle$ of a hypergraph $H$ is in component normal form (ComNF) if for each node $r \in T$, and for each child $s$ of $r$ there is exactly one $\left[B_{r}\right]$-component $C_{s}$ such that $V\left(T_{s}\right)=C_{s} \cup\left(B_{r} \cap B_{s}\right)$ holds. We say $C_{s}$ is the component associated with node $s$.

By the above definition, we know that in in a ComNF TD, every child $s$ of a node $r$ is associated with at most one $\left[B_{r}\right]$-component $C_{s}$. By the connectedness condition, also the converse is true, i.e., every $\left[B_{r}\right]$-component $C_{s}$ is associated with at most one child $s$. Note that (by applying the ideas of the transformation of HDs into the normal form of Reference [31]), every TD can be transformed in polynomial time into a TD in ComNF without increasing the width (more precisely, the bags in the resulting TD are subsets of the bags in the original TD).

Definition 4.4. Let $H$ be a hypergraph and let $\mathrm{S}$ be a family of subsets of $V(H)$. Let $\mathcal{T} \in \mathrm{CTD}(\mathrm{S})$ be a tree decomposition in ComNF. We say $\mathcal{T}$ is a ComNF candidate tree decomposition of $\mathrm{S}$. We denote by $\operatorname{ComCTD}(\mathrm{S})$ the set of all ComNF candidate tree decompositions of $H$.

Theorem 4.5. Let $H$ be a hypergraph and $\mathrm{S} \subseteq 2^{V(H)}$. There exists an algorithm that takes $H$ and $\mathrm{S}$ as an input and decides in polynomial time whether $\operatorname{ComCTD}(\mathrm{S}) \neq \emptyset$, and if so, return a $\mathcal{T} \in \operatorname{ComCTD}(\mathrm{S})$.

The intuition behind a polynomial-time algorithm for this problem is simple. Every parent/child relationship in a tree decomposition corresponds to a separator $S$ (the bag of the parent) and an $[S]$-component. It should therefore be enough to first enumerate all pairs $(S, C)$ of separators $S$ in $\mathrm{S}$ and $[S]$-components $C$, and then check if these pairs, which we will call blocks, can be combined to form a valid tree decomposition. Through the restriction to a specific set $\mathrm{S}$, that is part of the input, the number of blocks we have to consider is only polynomial in the input.

We are not aware of a proof of Theorem 4.5 in the literature. In light of the hardness result for the general case, we choose to present a full proof of the theorem in Appendix A even though it could be considered folklore. In particular, methods for subedge-based decompositions [32], as well as tree projections and their associated algorithms (see e.g., References [27, 29, 43]), are closely related to the ComNF CTD problem.

The restriction to component normal form will ultimately not restrict us in the following sections. For generalized and fractional hypertree width, ComNF can be enforced without increasing the width. Such a transformation can be found, e.g, in Reference [31] and as part of the proof of Lemma 5.9. Still, some care will be required in the enumeration of the candidate bags to guarantee that they allow for a decomposition in component normal form.

\section{TRACTABLE CASES OF GHD COMPUTATION}

As discussed in Section 1, we are interested in finding a realistic and non-trivial criterion on hypergraphs that makes the СНеск (GHD, $k$ ) problem tractable for fixed $k$. We thus propose here such a simple property, namely, the bounded intersection of two or more edges.

Definition 5.1. The intersection width iwidth $(H)$ of a hypergraph $H$ is the maximum cardinality of any intersection $e_{1} \cap e_{2}$ of two distinct edges $e_{1}$ and $e_{2}$ of $H$. We say that a hypergraph $H$ has the $i$-bounded intersection property $(i-B I P)$ if $i$ width $(H) \leq i$ holds.

Let $\mathscr{C}$ be a class of hypergraphs. We say that $\mathscr{C}$ has the bounded intersection property (BIP) if there exists some integer constant $i$ such that every hypergraph $H$ in $\mathscr{C}$ has the $i$-BIP. Class $\mathscr{C}$ has the logarithmically-bounded intersection property $(\log B I P)$ if for each of its elements $H$, $i$ width $(H)$ is $O(\log n)$, where $n=\|H\|$ denotes the size of $H$.

The BIP criterion properly generalizes bounded arity and is indeed non-trivial in the sense that there exist classes of unbounded ghw that enjoy the BIP. Among others this includes the classes 


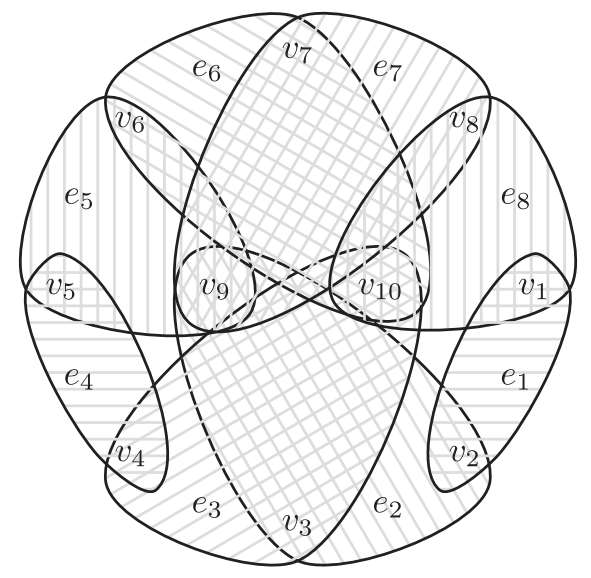

Fig. 5. Hypergraph $H_{0}$ from Example 5.3.

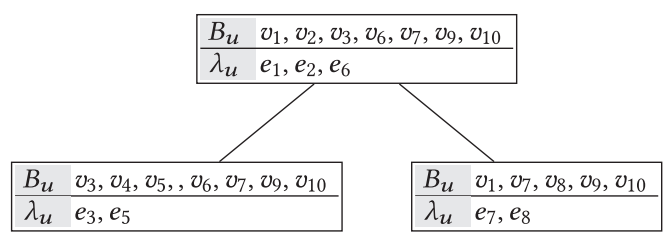

Fig. 6. HD of hypergraph $H_{0}$ in Figure 5.

of graphs, regular hypergraphs, and linear hypergraphs. Moreover, a recent empirical study [23] suggests that the overwhelming number of CQs enjoys the 2-BIP (i.e., one hardly joins two relations over more than two attributes). To allow for a yet bigger class of hypergraphs, the BIP can be relaxed as follows:

Definition 5.2. The c-multi-intersection width c-miwidth $(H)$ of a hypergraph $H$ is the maximum cardinality of any intersection $e_{1} \cap \cdots \cap e_{c}$ of $c$ distinct edges $e_{1}, \ldots, e_{c}$ of $H$. We say that a hypergraph $H$ has the $i$-bounded $c$-multi-intersection property (ic-BMIP) if $c$-miwidth $(H) \leq i$ holds.

Let $\mathscr{C}$ be a class of hypergraphs. We say that $\mathscr{C}$ has the bounded multi-intersection property (BMIP) if there exist constants $c$ and $i$ such that every hypergraph $H$ in $\mathscr{C}$ has the ic-BMIP. Class $\mathscr{C}$ of hypergraphs has the logarithmically-bounded multi-intersection property (LogBMIP) if there is a constant $c$ such that for the hypergraphs $H \in \mathscr{C}, c$-miwidth(H) is $O(\log n)$, where $n$ denotes the size of the hypergraph $H$.

Example 5.3. Figure 5 shows the hypergraph $H_{0}=\left(V_{0}, E_{0}\right)$ with $g h w\left(H_{0}\right)=2$ but $h w\left(H_{0}\right)=3$. (This example is from Reference [32], which, in turn, was inspired by work of Adler [3].) Figure 6 shows an HD of width 3, and Figure 7 shows GHDs of width 2 for the hypergraph $H_{0}$. The $i$ width and the 3-miwidth of $H_{0}$ is 1 . Starting from $c=4$, the $c$-miwidth is 0 .

The LogBMIP is the most liberal restriction on classes of hypergraphs introduced in Definitions 5.1 and 5.2. The main result in this section will be that the CHEck $(\mathrm{GHD}, k)$ problem with fixed $k$ is tractable for any class of hypergraphs satisfying this criterion. 


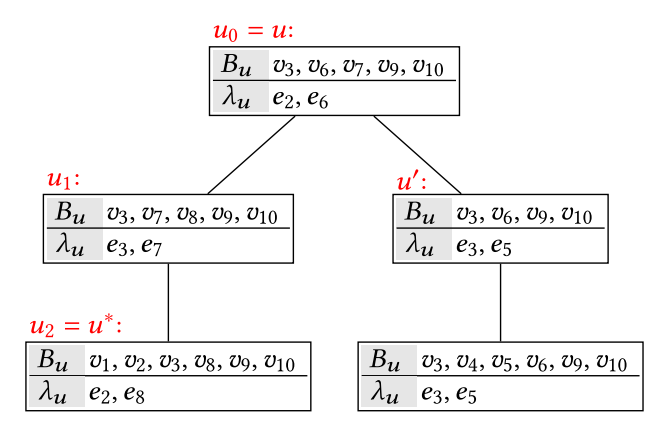

(a)

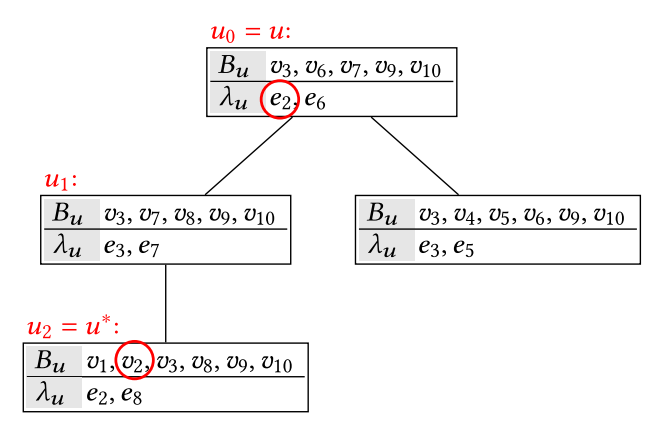

(b)

Fig. 7. (a) non bag-maximal vs. (b) bag-maximal GHD of hypergraph $H_{0}$ in Figure 5.

THEOREM 5.4. For every hypergraph class $\mathscr{C}$ that enjoys the LogBMIP, and for every constant $k \geq 1$, the $\operatorname{CHECK}(G H D, k)$ problem is tractable, i.e., given a hypergraph $H$, it is feasible in polynomial time to check $g h w(H) \leq k$ and, if so, to compute a GHD of width $k$ of $H$.

Our plan is to make use of Theorem 4.5 by computing appropriate sets $\mathrm{S}$ of candidate bags such that $\operatorname{ComCTD}(S) \neq \emptyset$ if and only if there exists a GHD with width at most $k$. Example 5.5 illustrates the main challenge that needs to be tackled to compute such sets of candidate bags. A bag $B_{u}$ in a GHD can be any subset of $B\left(\lambda_{u}\right)$ and choosing smaller subsets can decrease the width. At the same time, enumerating all subsets of $B\left(\lambda_{u}\right)$ is not an option if we are interested in classes of hypergraphs with unbounded rank. The main reason why the CHECK problem is tractable for HDs is that the additional special condition severely restricts the possible choices of $B_{u}$ for given $B\left(\lambda_{u}\right)$.

Example 5.5 (Example 5.3 continued). In Figure 7, we have two GHDs of width 2 of the hypergraph $H_{0}$ from Figure 5 . In the root $u_{0}$ of both GHDs, we have $v_{2} \in B\left(\lambda_{u_{0}}\right)$, since $v_{2} \in e_{2}$ but $v_{2} \notin B_{u_{0}}$. Hence, both GHDs violate the special condition in node $u_{0}$. However, if $v_{2}$ were added to $B_{u_{0}}$, then it can be seen that covering the edges $e_{1}, e_{2}, e_{7}, e_{8}$ below $u_{0}$ is no longer possible in a width 2 GHD. That is why the HD in Figure 6 has width 3.

We start by introducing a useful property of GHDs, which we will call bag-maximality. Let $\mathcal{H}=\left\langle T,\left(B_{u}\right)_{u \in T},\left(\lambda_{u}\right)_{u \in T}\right\rangle$ be a GHD of some hypergraph $H=(V(H), E(H))$. For each node $u$ in $T$, we have $B_{u} \subseteq B\left(\lambda_{u}\right)$ by definition of GHDs and, in general, $B\left(\lambda_{u}\right) \backslash B_{u}$ may be non-empty. We observe that it is sometimes possible to take some vertices from $B\left(\lambda_{u}\right) \backslash B_{u}$ and add them to $B_{u}$ without violating the connectedness condition. Of course, such an addition of vertices to $B_{u}$ does not violate any of the other conditions of GHDs. Moreover, it does not increase the width.

Definition 5.6. Let $\mathcal{H}=\left\langle T,\left(B_{u}\right)_{u \in T},\left(\lambda_{u}\right)_{u \in T}\right\rangle$ be a GHD of some hypergraph $H=(V(H), E(H))$. We call $\mathcal{H}$ bag-maximal if for every node $u$ in $T$, adding a vertex $v \in B\left(\lambda_{u}\right) \backslash B_{u}$ to $B_{u}$ would violate the connectedness condition.

It is easy to verify that if $H$ has a GHD of width $\leq k$, then it also has a bag-maximal GHD of width $\leq k$. However, since we want to build on the algorithm from Section 4 , we need to show that this also holds for bag-maximal ComNF GHDs. The problem here is that adding vertices to a bag $B_{u}$, to make it maximal, can change the set of $\left[B_{u}\right]$-components. Fortunately, we can reuse existing arguments on the existence of hypertree decompositions to show that if $H$ has a GHD of width $\leq k$, then it indeed also has a bag-maximal ComNF GHD of width $\leq k$.

We now carry over several properties of HDs from Reference [31]. An inspection of the corresponding proofs in Reference [31] reveals that these properties hold also in the generalized case. 
We thus state the following results below without explicitly "translating" the proofs of Reference [31] to the generalized setting. Note that Reference [31] deals with HDs and, therefore, in all decompositions considered there, the special condition holds. However, in Lemmas 5.7 and 5.8 below, the special condition is not needed.

We briefly recall the crucial notation for the following lemmas: For a set $V^{\prime} \subseteq V(H)$, we define $\operatorname{nodes}\left(V^{\prime}\right)=\left\{u \in T \mid B_{u} \cap V^{\prime} \neq \emptyset\right\}$. If we want to make explicit the decomposition $\mathcal{G}$, then we also write nodes $\left(V^{\prime}, \mathcal{G}\right)$ synonymously with nodes $\left(V^{\prime}\right)$. By further overloading the nodes operator, we also write $\operatorname{nodes}\left(T_{u}\right)$ or $\operatorname{nodes}\left(T_{u}, \mathcal{G}\right)$ to denote the nodes in a subtree $T_{u}$ of $T$, i.e., $\operatorname{nodes}\left(T_{u}\right)=$ $\operatorname{nodes}\left(T_{u}, \mathcal{G}\right)=\left\{v \mid v \in T_{u}\right\}$.

Lemma 5.7 (Lemma 5.2 from [31]). Consider an arbitrary GHD $\mathcal{H}=\left\langle T,\left(B_{u}\right)_{u \in T},\left(\lambda_{u}\right)_{u \in T}\right\rangle$ of a hypergraph $H$. Let $r$ be a node in $T$, let s be a child of $r$ and let $C$ be a $\left[B_{r}\right]$-component of $H$ such that $C \cap V\left(T_{s}\right) \neq \emptyset$. Then, $\operatorname{nodes}(C, \mathcal{H}) \subseteq \operatorname{nodes}\left(T_{s}\right)$.

Lemma 5.8 (Lemma 5.3 From [31]). Consider an arbitrary GHD $\mathcal{H}=\left\langle T,\left(B_{u}\right)_{u \in T},\left(\lambda_{u}\right)_{u \in T}\right\rangle$ of a hypergraph $H$. Let $r$ be a node in $T$ and let $U \subseteq V(H) \backslash B_{r}$ such that $U$ is $\left[B_{r}\right]$-connected. Then nodes $(U, \mathcal{H})$ induces a (connected) subtree of $T$.

Lemma 5.9. For every $G H D \mathcal{H}=\left\langle T,\left(B_{u}\right)_{u \in T},\left(\lambda_{u}\right)_{u \in T}\right\rangle$ of width $k$ of a hypergraph $H$, there exists a bag-maximal ComNF GHD $\mathcal{H}^{\prime}$ of $H$ of width $\leq k$. Moreover, for every node $u^{\prime}$ in $\mathcal{H}^{\prime}$ with cover $\lambda_{u^{\prime}}^{\prime}$, there exists a node $u$ in $\mathcal{H}$ such that $\lambda_{u}=\lambda_{u^{\prime}}^{\prime}$.

Proof. Start with a GHD $\mathcal{H}=\left\langle T,\left(B_{u}\right)_{u \in T},\left(\lambda_{u}\right)_{u \in T}\right\rangle$ of width $k$ of $H$. As long as there exists a node $u \in T$ and a vertex $v \in B\left(\lambda_{u}\right) \backslash B_{u}$, such that $v$ can be added to $B_{u}$ without destroying the GHD properties, select such a node $u$ and vertex $v$ arbitrarily and add $v$ to $B_{u}$. By exhaustive application of this transformation, a bag-maximal GHD of width $k$ of $H$ is obtained.

We proceed by restating a procedure from Reference [31] that fixes violations of the ComNF condition: For the bag-maximal GHD obtained above, assume that there exist two nodes $r$ and $s$ such that $s$ is a child of $r$, and the ComNF condition is violated for the pair, i.e., there does not exist a single $\left[B_{r}\right]$-component $C_{s}$ such that $V\left(T_{s}\right)=C_{s} \cup\left(B_{r} \cap B_{s}\right)$. Let $C_{1}, \ldots, C_{h}$ be all the $\left[B_{r}\right]$-components containing some vertex occurring in $V\left(T_{s}\right)$. Hence, $V\left(T_{s}\right) \subseteq\left(\bigcup_{j=1}^{h} C_{j} \cup B_{r}\right)$. For each $\left[B_{r}\right]$-component $C_{j}(1 \leq j \leq h)$, consider the set nodes $\left(C_{j}, \mathcal{H}\right)$. By Lemma $5.8, \operatorname{nodes}\left(C_{j}, \mathcal{H}\right)$ induces a subtree of $T$, and by Lemma $5.7, \operatorname{nodes}\left(C_{j}, \mathcal{H}\right) \subseteq \operatorname{nodes}\left(T_{s}\right)$. Hence, $\operatorname{nodes}\left(C_{j}, \mathcal{H}\right)$ induces in fact a subtree of $T_{s}$.

For each node $n \in \operatorname{nodes}\left(C_{j}, \mathcal{H}\right)$ define a new node $u_{n, j}$ and let $\lambda_{u_{n, j}}=\lambda_{n}$ and $B_{u_{n, j}}=B_{n} \cap$ $\left(C_{j} \cup B_{r}\right)$. Note that $B_{u_{n, j}} \neq \emptyset$, because by definition of nodes $\left(C_{j}, \mathcal{H}\right), B_{n}$ contains some vertex belonging to $C_{j}$. Let $N_{j}=\left\{u_{n, j} \mid n \in \operatorname{nodes}\left(C_{j}, \mathcal{H}\right)\right\}$ and, for any $C_{j}(1 \leq j \leq h)$, let $T_{j}$ denote the (directed) graph $\left(N_{j}, E_{j}\right)$ such that $u_{p, j}$ is a child of $u_{q, j}$ if and only if $p$ is a child of $q$ in $T . T_{j}$ is clearly isomorphic to the subtree of $T_{s}$ induced by nodes $\left(C_{j}, \mathcal{H}\right)$, hence $T_{j}$ is a tree as well.

Now transform the GHD $\mathcal{H}$ as follows: Delete the subtree $T_{s}$ from $T$ and attach to $r$ every tree $T_{j}$ for $1 \leq j \leq h$. In other words, we replace the subtree $T_{s}$ by a set of trees $\left\{T_{1}, \ldots, T_{h}\right\}$. By construction, $T_{j}$ contains a node $u_{n, j}$ for each node $n$ belonging to $\operatorname{nodes}\left(C_{j}, \mathcal{H}\right)(1 \leq j \leq h)$. Then, if we let children $(r)$ denote the set of children of $r$ in the new tree $T$ obtained after the transformation above, then it holds that for any $s^{\prime} \in \operatorname{children}(r)$, there exists a $\left[B_{r}\right]$-component $C$ of $H$ such that nodes $\left(T_{s^{\prime}}\right)=\operatorname{nodes}(C, \mathcal{H})$, and $V\left(T_{s^{\prime}}\right) \subseteq\left(C \cup B_{r}\right)$. We want to show $V\left(T_{s^{\prime}}\right)=$ $C \cup\left(B_{s^{\prime}} \cap B_{r}\right)$. For the " $\supseteq$ "-direction, we observe that $C \subseteq T_{s^{\prime}}$ clearly holds, since we have $C \subseteq T_{s}$ and the bags $B_{u_{n, j}}$ in $T_{s^{\prime}}$ were obtained from $B_{n}$ in $T_{s}$ as $B_{u_{n, j}}=B_{n} \cap\left(C_{j} \cup B_{r}\right)$ and we are considering the component $C=C_{j}$ here. Moreover, $B_{s^{\prime}} \cap B_{r} \subseteq B_{s^{\prime}} \subseteq V\left(T_{s^{\prime}}\right)$ clearly holds. Hence, we have $C \cup\left(B_{s^{\prime}} \cap B_{r}\right) \subseteq V\left(T_{s^{\prime}}\right)$. For the " $\subseteq$ "-direction, we conclude from $V\left(T_{s^{\prime}}\right) \subseteq C \cup B_{r}$ that also $V\left(T_{s^{\prime}}\right) \subseteq C \cup\left(V\left(T_{s^{\prime}}\right) \cap B_{r}\right)$ holds. Hence, it suffices to show that $\left(V\left(T_{s^{\prime}}\right) \cap B_{r}\right) \subseteq B_{s^{\prime}}$ holds. By 
connectedness, we have $V\left(T_{s}\right) \cap B_{r} \subseteq B_{s} \cap B_{r}$ and, therefore, also $V\left(T_{s^{\prime}}\right) \cap B_{r} \subseteq B_{s} \cap B_{r}$. Moreover, by construction, we have $B_{s^{\prime}}=B_{s} \cap\left(C \cup B_{r}\right)$ and, therefore $B_{s} \cap B_{r} \subseteq B_{s^{\prime}}$. We thus also arrive at $V\left(T_{s^{\prime}}\right) \subseteq C \cup\left(B_{s^{\prime}} \cap B_{r}\right)$.

It remains to show that $T_{s^{\prime}}$ is bag-maximal. Assume to the contrary that there exists a node $u_{n, j} \in T_{s^{\prime}}$ and a vertex $v \in B\left(\lambda_{u_{n, j}}\right) \backslash B_{u_{n, j}}$ such that $v$ can be added to $B_{u_{n, j}}$ without destroying the GHD properties. Recall that $B_{u_{n, j}}=B_{n} \cap\left(C_{j} \cup B_{r}\right)$ and $\lambda_{u_{n, j}}=\lambda_{n}$. Since $T$ was bag-maximal initially and from the construction (which only makes bags smaller), the only candidates for such a $v$ are those vertices $B_{n} \backslash\left(C_{j} \cup B_{r}\right)$ that got removed from the bag. However, all neighboring nodes are either $r$ or in $T_{s^{\prime}}$, which means their bags are subsets of $C_{j} \cup B_{r}$. Hence, no $v \in B_{n} \backslash\left(C_{j} \cup B_{r}\right)$ is contained in a neighbor of $u_{n, j}$ and adding it would break connectedness. Our newly constructed $T_{s^{\prime}}$ is therefore also bag-maximal.

Iterating this procedure for all ComNF violations will eventually produce a new GHD that is still bag-maximal and in ComNF. The second part of the statement follows from the observation that the construction never creates and new $\lambda_{u}$ sets, but only reuses covers from the input decomposition.

Example 5.10 (Example 5.5 continued). Clearly, the GHD in Figure 7(a) violates bag-maximality in node $u^{\prime}$, since the vertices $v_{4}$ and $v_{5}$ can be added to $B_{u^{\prime}}$ without violating any GHD properties. If we add $v_{4}$ and $v_{5}$ to $B_{u^{\prime}}$, then bag $B_{u^{\prime}}$ at node $u^{\prime}$ and the bag at its child node are the same, which allows us to delete one of the nodes. This results in the GHD given in Figure 7(b), which is bag-maximal. In particular, the vertex $v_{2}$ cannot be added to $B_{u_{0}}$ : Indeed, adding $v_{2}$ to $B_{u_{0}}$ would violate the connectedness condition, since $v_{2}$ is not in $B_{u_{1}}$ but in $B_{u_{2}}$.

For the following arguments, the reader is advised to be careful in distinguishing between the bag $B_{u}$ of a node $u$ and the set of vertices $B\left(\lambda_{u}\right)$ that are covered by the integral edge cover $\lambda_{u}$. Before we prove a crucial lemma, we introduce some useful notation:

Definition 5.11. Let $\mathcal{H}=\left\langle T,\left(B_{u}\right)_{u \in T},\left(\lambda_{u}\right)_{u \in T}\right\rangle$ be a GHD of a hypergraph $H$. Moreover, let $u$ be a node in $\mathcal{H}$ and let $e \in \lambda_{u}$ such that $e \backslash B_{u} \neq \emptyset$ holds. Let $u^{*}$ denote the node closest to $u$, such that $u^{*}$ covers $e$, i.e., $e \subseteq B_{u *}$. Then, we call the path $\pi=\left(u_{0}, u_{1}, \ldots, u_{\ell}\right)$ with $u_{0}=u$ and $u_{\ell}=u^{*}$ the critical path of $(u, e)$ denoted as $\operatorname{critp}(u, e)$.

Lemma 5.12. Let $\mathcal{H}=\left\langle T,\left(B_{u}\right)_{u \in T},\left(\lambda_{u}\right)_{u \in T}\right\rangle$ be a bag-maximal GHD of a hypergraph $H=(V(H)$, $E(H))$, let $u \in T, e \in \lambda_{u}$, and $e \backslash B_{u} \neq \emptyset$. Let $\pi=\left(u_{0}, u_{1}, \ldots, u_{\ell}\right)$ with $u_{0}=u$ be the critical path of $(u, e)$. Then the following equality holds:

$$
e \cap B_{u}=e \cap \bigcap_{j=1}^{\ell} B\left(\lambda_{u_{j}}\right) .
$$

Proof. " $\subseteq$ ": Given that $e \subseteq B_{u_{\ell}}$ and by the connectedness condition, $e \cap B_{u}$ must be a subset of $B_{u_{j}}$ for every $j \in\{1, \ldots, \ell\}$. Therefore, $e \cap B_{u} \subseteq e \cap \bigcap_{j=1}^{\ell} B\left(\lambda_{u_{j}}\right)$ holds.

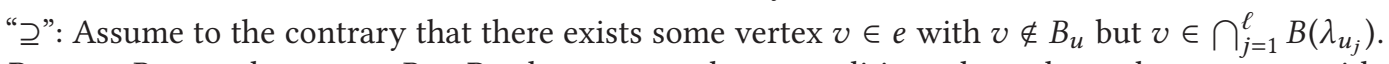
By $e \subseteq B_{u_{\ell}}$, we have $v \in B_{u_{\ell}}$. By the connectedness condition, along the path $u_{0}, \ldots, u_{\ell}$ with $u_{0}=u$, there exists $\alpha \in\{0, \ldots, \ell-1\}$, s.t. $v \notin B_{u_{\alpha}}$ and $v \in B_{u_{\alpha+1}}$. However, by the assumption, $v \in \bigcap_{j=1}^{\ell} B\left(\lambda_{u_{j}}\right)$ holds. In particular, $v \in B\left(\lambda_{u_{\alpha}}\right)$. Hence, we could safely add $v$ to $B_{u_{\alpha}}$ without violating the connectedness condition nor any other GHD condition. This contradicts the bagmaximality of $\mathcal{H}$.

Example 5.13 (Example 5.5 continued). Consider root node $u$ of the GHD in Figure 7(b). We have $e_{2} \in \lambda_{u}$ and $e_{2} \backslash B_{u}=\left\{v_{2}\right\} \neq \emptyset$. As $e_{2}$ is covered by $u_{2}$, the critical path of $\left(u, e_{2}\right)$ is $\pi=\left(u, u_{1}, u_{2}\right)$. It is easy to verify that $e_{2} \cap B_{u}=e_{2} \cap\left(e_{3} \cup e_{7}\right) \cap\left(e_{8} \cup e_{2}\right)=\left\{v_{3}, v_{9}\right\}$ indeed holds. 


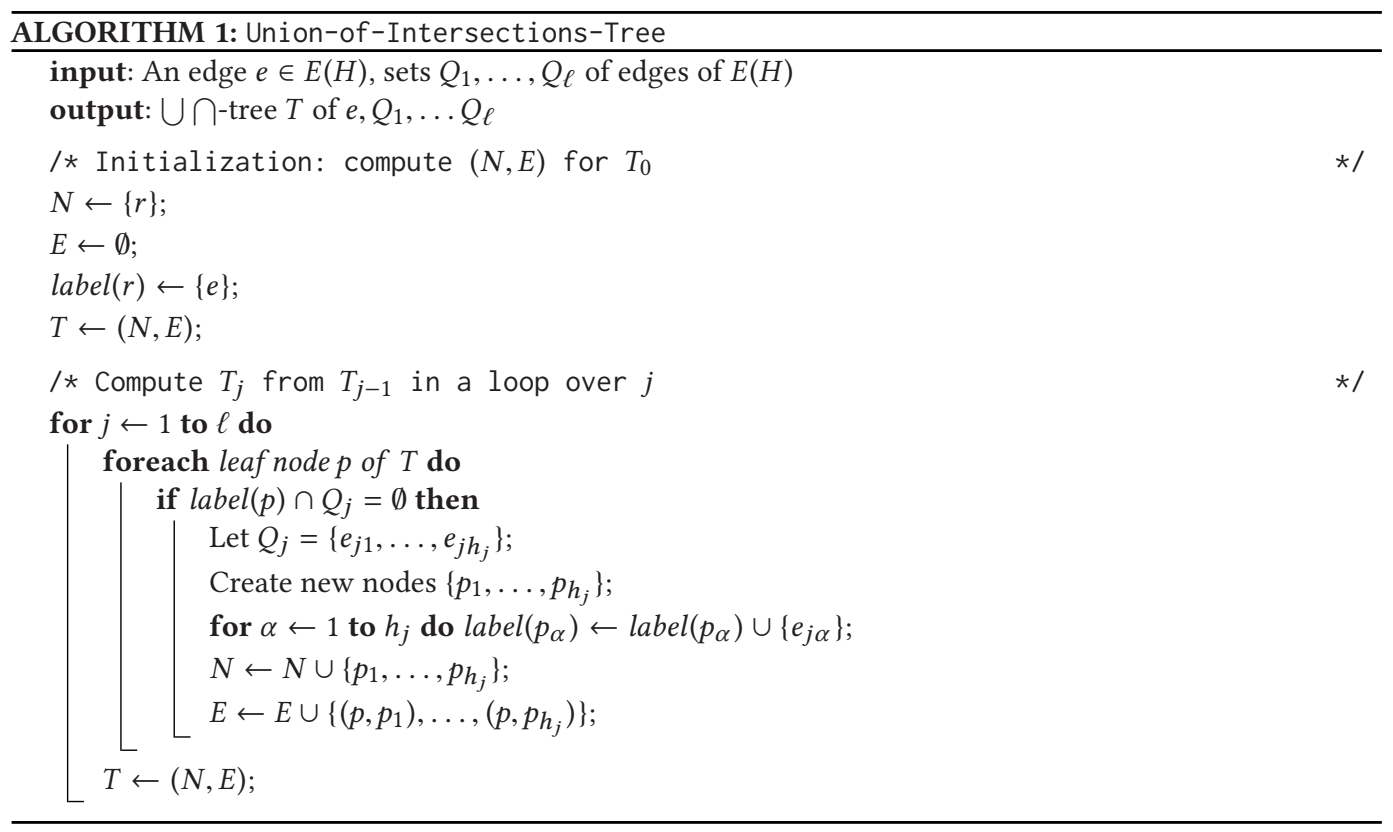

Lemma 5.12 characterizes the overlap of an edge with a bag as an intersection of unions. However, to utilize the proposed intersection constraints, we would prefer unions of intersections instead. A straightforward transformation from an intersection of unions to a union of intersections may introduce certain redundant terms that we would like to avoid for technical reasons. We therefore employ a particular transformation, via the $\cup \cap$-trees defined below, that avoids such redundant terms in the union.

Definition 5.14. Let $H$ be a hypergraph, $e$ an edge of $H$ and let $Q_{1}, \ldots, Q_{\ell}$ be sets of edges. The $\cup \cap$-tree $T$ of $e, Q_{1} \ldots, Q_{\ell}$ is the output of Algorithm 1 with inputs $e, Q_{1}, \ldots Q_{\ell}$. We refer to the set of all leaves of $T$ as leaves $(T)$.

Lemma 5.15. Let $H$ be a hypergraph, $e$ an edge of $H$ and let $Q_{1}, \ldots, Q_{\ell}$ be sets of edges. Let $T$ be the $\cup \cap$-tree of $e, Q_{1}, \ldots, Q_{\ell}$, then

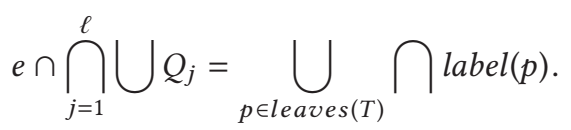

Proof. Proof is by induction over $\ell$. For $\ell=0$, we have leaves $(T)=\{r\}$ and label $(r)=e$ and the statement trivially holds. For $0 \leq j \leq \ell$, let $T_{j}$ denote the $\cup \cap$-tree of $e, Q_{1}, \ldots, Q_{j}$. Suppose the statement is true for $\ell-1$, then we observe the following equality:

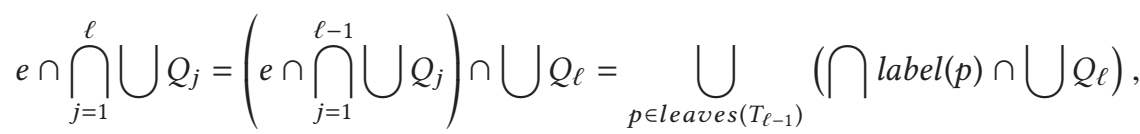

where the right equality follows from the induction hypothesis and distribution of $\cup Q_{\ell}$ over the union over the leaves of $T_{\ell-1}$. Now, consider a leaf $p \in$ leaves $\left(T_{\ell-1}\right)$. The construction of $T_{\ell}$ either adds new leaves $n e w_{p}$ as children of $p$, or $p$ remains a leaf in $T_{\ell}$. We claim that in the first case 
$\cap \operatorname{label}(p) \cap \cup Q_{\ell}=\bigcup_{p^{\prime} \in \text { new }_{p}} \cap \operatorname{label}\left(p^{\prime}\right)$ and in the second case, $\cap \operatorname{label}(p) \cap \cup Q_{\ell}=\bigcap \operatorname{label}(p)$. If the claim holds, then we have the following equality and the statement follows immediately:

$$
\bigcup_{p \in \text { leaves }\left(T_{\ell-1}\right)}\left(\bigcap \operatorname{label}(p) \cap \bigcup Q_{\ell}\right)=\bigcup_{p \in \text { leaves }\left(T_{\ell}\right)} \bigcap \operatorname{label}(p) .
$$

What is left is to verify the claim. The case where new children are added to $p$ is straightforward by distributivity as the label of each new child corresponds to a term of the union $(\cap \operatorname{label}(p) \cap$ $\left.e_{\ell 1}\right) \cup \cdots \cup\left(\cap \operatorname{label}(p) \cap e_{\ell h_{\ell}}\right)=\bigcap \operatorname{label}(p) \cap \bigcup Q_{\ell}$. If $p$ remains a leaf, then we have label $(p) \cap Q_{\ell} \neq \emptyset$. Thus, $\cap \operatorname{label}(p) \subseteq \cup Q_{\ell}$ and therefore $\cap \operatorname{label}(p) \cap \cup Q_{\ell}=\cap \operatorname{label}(p)$.

Throughout the rest of this article, we will be interested in how bags can be represented as combinations of edges. In particular, we will see that, under the various restrictions introduced at the beginning of this section, we are able to bound the representation of bags as unions of intersections of edges. After introducing some notation for such unions of intersections, we can show how the LogBMIP allows for a bounded representation of bags for GHDs. The main result then follows by using this representation to compute an appropriate set of candidate bags, to which Algorithm 2 from Section 4 can then be applied.

Definition 5.16. Let $H$ be a hypergraph. A $(q, p)$-set $X \subseteq V(H)$ is a set of the form $X=X_{1} \cup$ $\cdots \cup X_{q^{\prime}}$ with $q^{\prime} \leq q$ and where every $X_{i}$ is the intersection of at most $p$ edges. We will use $q$-set as shorthand for $(q, 1)$-set.

We will repeatedly make use of the fact that, by the idempotence of union and intersection, we can w.l.o.g. assume a $(q, p)$-set to be the union of exactly $q$ terms, each consisting of the intersection of exactly $p$ edges.

Lemma 5.17. Let $H$ be a hypergraph with c-miwidth $(H) \leq i$ and let $B_{1}, \ldots, B_{\ell}$ be $(q, 1)$-sets. For each $e \in E(H)$, there exists a $\left(q^{c-1}, c\right)$-set I and a subedge $e^{\prime} \subseteq e$ with $\left|e^{\prime}\right| \leq i q^{c}$, such that

$$
e \cap \bigcap_{j=1}^{\ell} B_{j}=I \cup e^{\prime}
$$

Furthermore, $e^{\prime}$ is the union of at most $q^{c}$ subsets of intersections of exactly $c$ edges.

Proof. For $j \in[\ell]$, fix a set of edges $Q_{j}=\left\{e_{j 1}, \ldots, e_{j q}\right\}$ such that $B_{j}=e_{j 1} \cup \cdots \cup e_{j q}$. Let $T$ be the $\bigcup \cap$-tree of $e, Q_{1}, \ldots, Q_{\ell}$. For a node $p$ of $T$, we refer to the number of edges in the path from the root to $p$ as the depth of $p$, or $\operatorname{depth}(p)$. Note that, by construction, $|\operatorname{label}(p)|=\operatorname{depth}(p)+1$ for each node $p$ in $T$. We consider the following partition of leaves $(T)$ : Let $S M A L L$ contain all the leaves of $T$ at depth at most $c-1$ and, conversely, let FULL be the set of leaves at depth at least $c$.

By Lemma 5.15, $e \cap \bigcap_{j=1}^{\ell} B_{j}=\left(\bigcup_{p \in S M A L L} \cap \operatorname{label}(p)\right) \cup\left(\bigcup_{p \in F U L L} \cap \operatorname{label}(p)\right)$. Hence, to prove the lemma, it suffices to show that $\bigcup_{p \in S M A L L} \cap \operatorname{label}(p)$ is a $\left(q^{c-1}, c\right)$-set and that $\mid \bigcup_{p \in F U L L}$ $\cap \operatorname{label}(p) \mid \leq i q^{c}$ holds.

Claim A. $\cup_{p \in S M A L L} \cap \operatorname{label}(p)$ is a $\left(q^{c-1}, c\right)$-set.

Proof of Claim A. Since each of the sets $Q_{j}$ has at most $q$ members, every node in $T$ has at most $q$ children. Hence, there are at most $q^{c-1}$ leaves at depth $\leq c-1$ and therefore $|S M A L L| \leq q^{c-1}$. Furthermore, we have $|\operatorname{label}(p)|=\operatorname{depth}(p)+1 \leq c$, i.e., each intersection has at most $c$ terms. $\diamond$

Claim B. $\left|\cup_{p \in F U L L} \cap \operatorname{label}(p)\right| \leq i q^{c}$.

Proof of Claim B. First, observe that for each $p \in F U L L$, there exists a node $p^{\prime}$ in $T$ at depth $c$ such that $\operatorname{label}(p) \supseteq \operatorname{label}\left(p^{\prime}\right)$ and therefore also $\bigcap \operatorname{label}(p) \subseteq \bigcap \operatorname{label}\left(p^{\prime}\right)$. Note that there are at most $q^{c}$ nodes $p^{\prime}$ at depth $c$. Furthermore, because $\left|\operatorname{label}\left(p^{\prime}\right)\right|=c$ and we assume $c$-miwidth $(H) \leq i$, 
it holds that $\left|\cap \operatorname{label}\left(p^{\prime}\right)\right| \leq i$. In total, we thus have that $\bigcup_{p \in F U L L} \cap \operatorname{label}(p)$ is a union of sets $X_{p}$ such that each $X_{p}$ is the subset of one out of at most $q^{c}$ vertex sets, and each of these vertex sets has cardinality at most $i$.

For a given edge cover $\lambda_{u}$ and arbitrary edge $e \in E(H)$ with $\lambda_{u}(e)=1$, Lemma 5.12 gives us a representation of $e \cap B_{u}$ of the form $e \cap \bigcap_{j=1}^{\ell} B\left(\lambda_{u_{j}}\right)$. Clearly, the sets $B\left(\lambda_{u_{j}}\right)$ are $(k, 1)$-sets, i.e., unions of (up to) $k$ edges. We can therefore apply Lemma 5.17 by taking $B_{j}=B\left(\lambda_{u_{j}}\right)$ and $q=k$ to get a representation of the form $I \cup e^{\prime}$ for each of the possible subedges $e \cap B_{u}$ that may ever be used in a bag-maximal ComNF GHD. This idea is formalized in the following lemma, where we identify a polynomially big family of vertex sets $S \subseteq 2^{V(H)}$, such that the bags of any bag-maximal ComNF GHD of $H$ must be a member of this family:

LEMmA 5.18. Let $H$ be a hypergraph with $c$-miwidth $(H) \leq b$, let $n=\|H\|$ be the size of $H$, and fix an integer $k>0$. There exists a set $\mathrm{S} \subseteq 2^{V(H)}$, which can be computed in $2^{b \cdot f_{1}(c, k)} n^{f_{2}(c, k)}$ time, such that $\operatorname{ComCTD}(\mathrm{S}) \neq \emptyset$ if and only if $g h w(H) \leq k$.

Furthermore, for any bag-maximal ComNF GHD $\left\langle T,\left(B_{u}\right)_{u \in T},\left(\lambda_{u}\right)_{u \in T}\right\rangle$ of $H$ of width $\leq k$, we have $\left\langle T,\left(B_{u}\right)_{u \in T}\right\rangle \in \operatorname{ComCTD}(\mathrm{S})$.

Proof. Let $m=|E(H)|$. We define the following sets:

$\mathcal{I}=\left\{I \mid I\right.$ is a $\left(k^{c-1}, c\right)$-set $\}$,

$C=\left\{e^{\prime} \mid\right.$ there exist distinct $e_{1}, \ldots, e_{c} \in E(H)$, such that $\left.e^{\prime} \subseteq e_{1} \cap \cdots \cap e_{c}\right\}$,

$S u b=\left\{I \cup \bigcup_{j=1}^{k^{c}} C_{j} \mid I \in \mathcal{I}, C_{1}, \ldots, C_{k^{c}} \in C\right.$, and $I \cup \bigcup_{j=1}^{k^{c}} C_{j} \subseteq e$ for some $\left.e \in E(H)\right\}$.

By construction, $S u b$ contains only $E(H)$ and subedges of $H$. There are no more than $m^{c k^{c-1}}$ possible $\left(k^{c-1}, c\right)$ sets. Also, by the condition $c$-miwidth $(H) \leq b$, we have $|C| \leq 2^{b} m^{c}$ and, therefore, $S u b$ has at most $m^{c k^{c-1}} m^{c k^{c}} 2^{b k^{c}}$ elements. Hence, $S u b$ can be computed in $\left.2^{b \cdot f(c, k)} n^{f^{\prime}(c, k)}\right)$ time for some functions $f$ and $f^{\prime}$ by straightforward enumeration. We can now construct our desired set $\mathrm{S}$ as the set of all unions of up to $k$ elements of $S u b$. It follows that the construction of S is possible in $2^{b \cdot f_{1}(c, k)} n^{f_{2}(c, k)}$ time for some functions $f_{1}$ and $f_{2}$.

It remains to show that this $\mathrm{S}$ indeed has the property that (1) ComCTD(S) $\neq \emptyset$ if and only if $g h w(H) \leq k$ and (2) for any bag-maximal ComNF GHD $\left\langle T,\left(B_{u}\right)_{u \in T},\left(\lambda_{u}\right)_{u \in T}\right\rangle$ of $H$ of width $\leq k$, we have $\left\langle T,\left(B_{u}\right)_{u \in T}\right\rangle \in \operatorname{ComCTD}(\mathrm{S})$.

First, assume ComCTD $(S) \neq \emptyset$. Then there exists a TD of $H$ where each bag is in $\mathrm{S}$ and therefore a union of $k$ subedges of $H$. Hence, every bag of the TD can also be covered by $k$ edges of $H$ and thus can clearly be turned into a GHD of width at most $k$.

Now, assume $g h w(H) \leq k$. Let $\mathcal{H}=\left\langle T,\left(B_{u}\right)_{u \in T},\left(\lambda_{u}\right)_{u \in T}\right\rangle$ be a bag-maximal ComNF GHD of width at most $k$ and let $u$ be a node of $T$. By Lemma 5.9, such a GHD always exists if $g h w(H) \leq k$. W.l.o.g., we assume that $B\left(\lambda_{u}\right)=e_{1} \cup \cdots \cup e_{k}$ and, therefore, also

$$
B_{u}=B_{u} \cap B\left(\lambda_{u}\right)=\left(B_{u} \cap e_{1}\right) \cup \cdots \cup\left(B_{u} \cap e_{k}\right) .
$$

We will show that $B_{u} \cap e_{j} \in S u b$ for each $j \in[k]$ and therefore also $B_{u} \in \mathrm{S}$. The case where $e_{j} \cap B_{u}=e_{j}$ is trivial as $e_{j} \in E(H) \subseteq S u b$. So, let $e_{j}$ be any edge from this representation of $B\left(\lambda_{u}\right)$ where $e_{j} \cap B_{u} \neq e_{j}$. By Lemma 5.12, the following equality holds for the critical path $\left(u, u_{1}, \ldots, u_{\ell}\right)$ of $\left(u, e_{j}\right)$ :

$$
e_{j} \cap B_{u}=e_{j} \cap \bigcap_{j=1}^{\ell} B\left(\lambda_{u_{j}}\right) .
$$

By assumption, every such $B\left(\lambda_{u_{j}}\right)$ is a $k$-set and hence, by Lemma 5.17 , we know that $e_{j} \cap$ $\bigcap_{j=1}^{\ell} B\left(\lambda_{u_{j}}\right)$ is precisely the union of an element of $\mathcal{I}$ and at most $k^{c}$ sets from $C$, i.e., $e_{j} \cap B_{u} \in$ 
Sub. Since the choice of $u$ was arbitrary, every bag of $\mathcal{H}$ is contained in $\mathrm{S}$ and we have $\mathcal{H} \in$ $\operatorname{ComCTD}(\mathrm{S})$.

Proof of Theorem 5.4: We assume that $\mathscr{C}$ enjoys the LogBMIP, i.e., for every $H \in \mathscr{C}$, we have $c$-miwidth $(H) \leq a \log n$. To solve the Снеск(GHD, k) problem, we can then simply compute, in polynomial time, the set $\mathrm{S}$ from Lemma 5.18 and decide whether $\operatorname{ComCTD}(\mathrm{S}) \neq \emptyset$. By Theorem 4.5 this is also tractable and, therefore, so is the whole procedure.

We have defined in Section 1 the degree $d$ of a hypergraph $H$. We now consider hypergraphs of bounded degree.

Definition 5.19. We say that a hypergraph $H$ has the $d$-bounded degree property $(d-B D P)$ if $\operatorname{degree}(H) \leq d$ holds.

Let $\mathscr{C}$ be a class of hypergraphs. We say that $\mathscr{C}$ has the bounded degree property (BDP) if there exists a constant $d$ such that every hypergraph $H$ in $\mathscr{C}$ has the $d$-BDP.

The class of hypergraphs of bounded degree is an interesting special case of the class of hypergraphs enjoying the BMIP. Indeed, suppose that each vertex in a hypergraph $H$ occurs in at most $d$ edges for some constant $d$. Then the intersection of $d+1$ hyperedges is always empty. The following corollary is thus immediate:

Corollary 5.20. For every class $\mathscr{C}$ of hypergraphs of bounded degree, for each constant $k$, the problem $\operatorname{CHECK}(G H D, k)$ is tractable.

In case of the BMIP, the upper bound on S in the proof of Lemma 5.18, is $2^{f(i, k, c)} m^{g(k, c)}$ for some function $g$. Recall from Theorem 4.5 that $\operatorname{ComCTD}(\mathbf{S}) \neq \emptyset$ can be decided in time complexity that is polynomial in $|\mathbf{S}| \cdot|V(H)|$. We thus get the following parameterized complexity result:

TheOREM 5.21. For constants $k$ and $c$, the CHECK $(G H D, k)$ problem is fixed-parameter tractable w.r.t. the parameter $i$ for hypergraphs enjoying the ic-BMIP, i.e., in this case, $C H E C K(G H D, k)$ can be solved in time $O(h(i) \cdot$ poly $(n))$, where $h(i)$ is a function depending on the intersection width $i$ only and poly $(n)$ is a function that depends polynomially on the size $n=\|H\|$ of the given hypergraph $H$.

For practical purposes, this is of particular interest in case of the BIP, i.e., $c=2$. Here, the construction of $S u b$ can be significantly simplified, since, for any critical path $\operatorname{critp}(u, e)=$ $\left(u, u_{1}, \ldots, u_{\ell}\right)$, it is enough to consider only one step, i.e., $e \cap B_{u} \subseteq e \cap B\left(\lambda_{\hat{u}}\right)=e \cap\left(e_{\hat{u} 1} \cup \cdots \cup e_{\hat{u} k}\right)$, for some node $\hat{u}$, such that all edges $e_{\hat{u} j}$ are distinct from $e$. This is the case for the following reasons: First, by connectedness, $e \cap B_{u} \subseteq e_{u_{j} 1} \cup \cdots \cup e_{u_{j} k}$ for every node $u_{j}$ on this critical path. And second, there is at least one node $\hat{u}$ on this critical path such that $e \notin \lambda_{\hat{u}}$. Indeed, if $e$ were present in $\lambda_{u_{j}}$ for every $j$, then we could add $e$ to every bag $B_{u_{j}}$ on the critical path without violating the connectedness condition. This would contradict the assumption of bag-maximality.

By the BIP, we have $\left|e \cap e_{\hat{u} j}\right| \leq i$ for every $j$ and, therefore, we can simply compute all the subsets of $e \cap B\left(\lambda_{\hat{u}}\right)$ in polynomial time to construct $S u b$ as follows:

$$
S u b=E(H) \cup \bigcup_{e \in E(H)}\left(\bigcup_{e_{1}, \ldots, e_{j} \in(E(H) \backslash\{e\}), j \leq k} 2^{\left(e \cap\left(e_{1} \cup \cdots \cup e_{j}\right)\right)}\right),
$$

i.e., $S u b$ contains $E(H)$ and all subsets of intersections of edges $e \in E(H)$ with unions of $\leq k$ edges of $H$ different from $e$. Here, the BIP is only used implicitly in the sense that we can be sure that the above expression is computable in polynomial time, because $\left|e \cap\left(e_{1} \cup \cdots \cup e_{j}\right)\right| \leq j i \leq k i$ holds by the BIP. The explicit use of the BIP allows for a yet simpler (more coarse-grained) way to define Sub as follows:

$$
S u b=E(H) \cup\left\{e^{\prime} \mid e^{\prime} \subseteq e \text { for some } e \in E(H) \text { and }\left|e^{\prime}\right| \leq k i\right\} .
$$




\section{TRACTABLE CASES OF FHD COMPUTATION}

\subsection{Initial Considerations and Lemmas}

In Section 5, we have shown that under certain conditions (with the BIP and BDP as most specific and the LogBMIP as most general conditions) the problem of computing a GHD of width $\leq k$ can be reduced to finding a ComNF candidate tree decomposition for an appropriate set of candidate bags. The key to this problem reduction was to enumerate a set of subedges, which allowed us to enumerate all bags of possible bag-maximal GHDs of width $\leq k$. When trying to carry over these ideas from GHDs to FHDs, we encounter two major challenges: Can we adapt our approach for GHDs to work with FHDs? And is it possible to find bounded representations of all the sets of vertices that can be fractionally covered with weight $\leq k$ ?

For the second challenge, recall from the GHD-case that the possible bags $B\left(\lambda_{u}\right)$ could be easily computed from the given set of subedges, since each $\lambda_{u}$ can choose at most $k$ subedges. In contrast, for a fractional cover $\gamma_{u}$, we do not have such a bound on the number of edges with non-zero weight. It is easy to exhibit a family $\left(H_{n}\right)_{n \in \mathbb{N}}$ of hypergraphs where it is advantageous to have unbounded $\operatorname{supp}\left(\gamma_{n}\right)$ even if $\left(H_{n}\right)_{n \in \mathbb{N}}$ enjoys the BIP, as the following example illustrates:

Example 6.1. Consider the family $\left(H_{n}\right)_{n \in \mathbb{N}}$ of hypergraphs with $H_{n}=\left(V_{n}, E_{n}\right)$ defined as follows:

$V_{n}=\left\{v_{0}, v_{1}, \ldots, v_{n}\right\}$,

$E_{n}=\left\{\left\{v_{0}, v_{i}\right\} \mid 1 \leq i \leq n\right\} \cup\left\{\left\{v_{1}, \ldots, v_{n}\right\}\right\}$.

Clearly iwidth $\left(H_{n}\right)=1$, but an optimal fractional edge cover of $H_{n}$ is obtained by the following mapping $\gamma$ with $\operatorname{supp}(\gamma)=E_{n}$ :

$$
\begin{aligned}
& \gamma\left(\left\{v_{0}, v_{i}\right\}\right)=1 / n \text { for each } i \in\{1, \ldots, n\} \text { and } \\
& \gamma\left(\left\{v_{1}, \ldots, v_{n}\right\}\right)=1-(1 / n)
\end{aligned}
$$

such that weight $(\gamma)=2-(1 / n)$, which is optimal in this case.

Further below, we show that, for cases where the second challenge can be resolved (i.e., we can establish an upper bound on $\operatorname{supp}\left(\gamma_{u}\right)$ for all nodes $u$ in an FHD), the check-problem of FHDs can be essentially reduced to the GHD case. The respective Lemma 6.5 is thus the crucial tool for the remainder of this article. Before we move on to proving the crucial lemma, we need some further tools. Similarly as in the GHD-case, we will have to deal with unions of intersections of edges also in the FHD-case. The following definition and the accompanying two lemmas will be convenient for this purpose:

Definition 6.2. For a hypergraph $H$, we write $H^{\cap}$ for the closure of $H$ under intersection of edges.

It will be important to observe that adding subedges does not change the fractional hypertree width of a hypergraph. Every FHD of $H$ is still an FHD of $H^{\cap}$ and every FHD of $H^{\cap}$ can be easily transformed into an FHD of $H$. It follows that we always have $f h w(H)=f h w\left(H^{\cap}\right)$.

LEMMA 6.3. Let $H$ be a hypergraph and $\gamma$ a fractional edge cover of a set $S$ of vertices. Then $B(\gamma)$

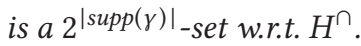

Proof. Let us call a subset $E \subseteq \operatorname{supp}(\gamma)$ full if $\sum_{e \in E} \gamma(e) \geq 1$ and let FS contain all the full subsets. For every $E \in \mathrm{FS}$, we have $\bigcap E \subseteq B(\gamma)$ and it is straightforward to verify the following equality:

$$
B(\gamma)=\bigcup_{E \in \mathrm{FS}} \bigcap E
$$

It is then enough to observe that $\bigcap E \in E\left(H^{\cap}\right)$ and that there are at most $2^{|\operatorname{supp}(\gamma)|}$ full subsets. 
Lemma 6.4. Let $H$ be a hypergraph with c-miwidth $(H) \leq i$. Then $H^{\cap}$ can be computed in polynomial time for fixed $c$ and $i$. Moreover, $2^{c}$-miwidth $\left(H^{\cap}\right) \leq i$ holds.

Proof. First, note that if $\left|E\left(H^{\cap}\right)\right| \leq 2^{c}$, then also $|E(H)| \leq 2^{c}$ and we can trivially compute $H^{\cap}$ in polynomial time by simply computing all possible intersections of edges in $E(H)$. Moreover, in this case, the condition $2^{c}$-miwidth $\left(H^{\cap}\right) \leq i$ is void, since there are no distinct $2^{c}$ edges in $H^{\cap}$.

Now let $\alpha=2^{c}$ and consider an intersection $I$ of $\alpha$ distinct edges of $H^{\cap}$ of the form $I=e_{1}^{\prime} \cap \cdots \cap e_{\alpha}^{\prime}$, where $e_{j}^{\prime} \in E\left(H^{\cap}\right)$ for $j \in[\alpha]$. Each $e_{j}^{\prime} \in E\left(H^{\cap}\right)$ is an intersection of edges from $E(H)$, i.e., there exists $\mathcal{E}_{j} \subseteq E(H)$ such that $e_{j}^{\prime}=\bigcap_{e \in \mathcal{E}_{j}}$ e. Clearly, $I=\bigcap\left(\bigcup_{j=1}^{\alpha} \mathcal{E}_{j}\right)$.

We claim that $\left|\bigcup_{j=1}^{\alpha} \mathcal{E}_{j}\right| \geq c$, i.e., $I$ is the intersection of at least $c$ edges from $E(H)$. Indeed, with less than $c$ distinct edges, there could only be less than $2^{c}-1$ non-empty sets $\mathcal{E}_{j}$. It thus follows that $I$ is a subset of an intersection of at least $c$ edges of $E(H)$. Thus, by $c$-miwidth $(H) \leq i$, we conclude that $|I| \leq i$ holds. Hence, also in the case $\left|E\left(H^{\cap}\right)\right|>2^{c}$, the condition $2^{c}$-miwidth $\left(H^{\cap}\right) \leq i$ holds.

It remains to show that $E\left(H^{\cap}\right)$ can be computed from $E(H)$ in polynomial time: let $m=|E(H)|$. Then there are less than $m^{c}$ intersections of less than $c$ distinct edges from $E(H)$ and these intersections can clearly be computed in polynomial time. To compute also the set of intersections of at least $c$ edges from $E(H)$, we proceed as follows: We first compute the set $\mathcal{I}_{0}$ of intersections of $c$ edges. Then, for every $j \in[m-c]$, we compute the set $\mathcal{I}_{j}$ of intersections of $\alpha$ edges from $E(H)$ with $\alpha \in\{c, \ldots, c+j\}$. By $c$-miwidth $(H) \leq i$, we know that $\left|\mathcal{I}_{j}\right| \leq 2^{i} m^{c}$ holds for every $j \in[m-c]$. Hence, all these intersections can clearly be computed in polynomial time (for fixed $c$ and $i$ ).

Now that these properties of $H^{\cap}$ are verified, we can state the main technical lemma for this section. The main algorithmic results of this section are all based on the following statement:

LEMma 6.5. Let $H$ be a hypergraph with $c$-miwidth $(H) \leq i$ for constants $c$ and $i$, and fix a rational $k$ and integer $q \geq 1$. There exists a polynomial time algorithm that decides, whether there exists an FHD $\mathcal{F}$ of width $k$, such that for each node $u$ of the decomposition with cover $\gamma_{u}$, it holds that $\left|\operatorname{supp}\left(\gamma_{u}\right)\right| \leq q$.

Proof. Let $\rho_{q, H}^{*}(X)$ denote the minimal weight of all fractional edge covers $\gamma$ (in $H$ ) such that $X \subseteq B(\gamma)$ and $|\operatorname{supp}(\gamma)| \leq q$. Using this function, we will define a set of candidate bags $\mathrm{S}$ such that $\operatorname{ComCTD}(\mathrm{S}) \neq \emptyset$ if and only if there exists an FHD $\mathcal{F}$ as in the statement of the lemma. We demonstrate that $\mathrm{S}$ can be computed in polynomial time (for fixed $c, i, k$, and $q$ ) and the statement then follows from Theorem 4.5.

To define this set $\mathrm{S}$, we first use the result for the GHD-case: Let $\mathrm{S}_{g h d}$ be the set from Lemma 5.18 such that $\operatorname{ComCTD}\left(\mathrm{S}_{g h d}\right) \neq \emptyset$ if and only if $g h d\left(H^{\cap}\right) \leq 2^{q}$. By Lemma 6.4, $H^{\cap}$ can be computed in polynomial time, and thus by Lemma 5.18, we have that such an $\mathrm{S}_{g h d}$ exists and can also be computed in polynomial time. We can then obtain the set $\mathrm{S}=\left\{S \in \mathrm{S}_{g h d} \mid \rho_{q, H}^{*}(S) \leq k\right\}$. The function $\rho_{q, H}^{*}(S)$ can be computed, for each set $S \in \mathrm{S}_{g h d}$, by solving a linear program for every combination of $q$ edges in $E(H)$, i.e., by solving $\left(\begin{array}{c}|E(H)| \\ q\end{array}\right)$ linear programs. Hence, also $\mathrm{S}$ can be computed in polynomial time.

We now argue that ComCTD(S) behaves as claimed in the beginning of the proof. Suppose $\operatorname{ComCTD}(\mathrm{S}) \neq \emptyset$, and recall that $H^{\cap}$ has the same vertices as $H$. Then clearly there is a TD where every bag has $\rho_{q, H}^{*}$ at most $k$ and, therefore, there is a width $k$ FHD where every cover has support bounded by $q$.

For the other direction, suppose $\mathcal{F}=\left\langle T,\left(B_{u}\right)_{u \in T},\left(\gamma_{u}\right)_{u \in T}\right\rangle$ is an FHD of $H$ with width $k$, such that for each node $u$ of $\mathcal{F}$ with cover $\gamma_{u}$, it holds that $\left|\operatorname{supp}\left(\gamma_{u}\right)\right| \leq q$. By Lemma 6.3, there exists an FHD $\mathcal{F}^{\prime}=\left\langle T,\left(B_{u}\right)_{u \in T},\left(\gamma_{u}^{\prime}\right)_{u \in T}\right\rangle$ of $H^{\cap}$ with the same width, such that $B\left(\gamma_{u}^{\prime}\right)=B\left(\gamma_{u}\right)$ and $B\left(\gamma_{u}^{\prime}\right)$ is a $2^{q}$-set. 
That is, each $B\left(\gamma_{u}^{\prime}\right)$ is of the form $e_{u, 1} \cup \cdots \cup e_{u, \ell_{u}}$ with $\ell_{u} \leq 2^{q}$. Now let $\lambda_{u}=\left\{e_{u, 1}, \ldots, e_{u, \ell_{u}}\right\}$ for each $u \in T$. It is easy to see that $\mathcal{H}=\left\langle T,\left(B_{u}\right)_{u \in T},\left(\lambda_{u}\right)_{u \in T}\right\rangle$ is a GHD of $H^{\cap}$ with $g h w(\mathcal{H}) \leq$ $2^{q}$. Note that $\mathcal{H}$ is not necessarily bag-maximal or in ComNF. However, following the procedure described in Lemma 5.9, there exists a bag-maximal ComNF $\mathcal{H}^{\prime}=\left\langle T^{\prime},\left(B_{u}^{\prime}\right)_{u \in T^{\prime}},\left(\lambda_{u}^{\prime}\right)_{u \in T^{\prime}}\right\rangle$ of $H^{\cap}$ with $g h w\left(\mathcal{H}^{\prime}\right) \leq 2^{q}$ and therefore $\left\langle T^{\prime},\left(B_{u}^{\prime}\right)_{u \in T^{\prime}}\right\rangle \in \operatorname{ComCTD}\left(\mathrm{S}_{g h d}\right)$.

Now recall that the transformation into a bag-maximal ComNF $\mathcal{H}^{\prime}$ from Lemma 5.9 never changes the covers in the GHD, i.e., if $\lambda$ is the cover of some node in $\mathcal{H}^{\prime}$, then that same $\lambda$ already was a cover of some node in $\mathcal{H}$. That is, every bag of $\mathcal{H}^{\prime}$ is a subset of set $\bigcup \lambda_{u}$ for some node $u$ in $\mathcal{H}$. Recall that we have $\cup \lambda_{u}=B\left(\gamma_{u}^{\prime}\right)$ where $\gamma_{u}^{\prime}$ is the fractional cover in $\mathcal{F}^{\prime}$. Hence, every bag of $\mathcal{H}^{\prime}$ is also a subset of some $B\left(\gamma_{u}\right)$ in $\mathcal{F}$. Therefore, $\left\langle T^{\prime},\left(B_{u}^{\prime}\right)_{u \in T^{\prime}}\right\rangle$ is in ComNF, each of its bags is in $\mathrm{S}_{g h d}$ and can be fractionally covered (in $H$ ) with weight $k$ and at most $q$ edges in the support, i.e., $\left\langle T^{\prime},\left(B_{u}^{\prime}\right)_{u \in T^{\prime}}\right\rangle \in \operatorname{ComCTD}(\mathrm{S})$.

To apply Lemma 6.5, we will have to prove that an appropriate constant $q$ indeed exists. The following two subsections will show that such a constant indeed exists for classes of either bounded intersection width or bounded degree. Furthermore, in Section 7, we will also use the lemma to derive an approximation result for classes that exhibit the BMIP.

\subsection{Computing FHDs under Bounded Degree}

First, we will investigate the situation for classes of hypergraphs whose degree is bounded by a constant $d$. We show that a fractional edge cover $\gamma$ of a hypergraph $H$ has support $\sup p(\gamma)$ bounded by a constant that depends only on weight $(\gamma)$ and $d$. From there, tractability follows from Lemma 6.5.

THEOREM 6.6. For every hypergraph class $\mathscr{C}$ that has bounded degree, and for every rational constant $k \geq 1$, the $\operatorname{CHECK}(F H D, k)$ problem is tractable, i.e., given a hypergraph $H \in \mathscr{C}$, it is feasible in polynomial time to check $f h w(H) \leq k$ and, if so, to compute an FHD of width $k$ of $H$.

For this result, we need to introduce, analogously to edge-weight functions and edge covers in Section 2, the notions of vertex-weight functions and vertex covers.

Definition 6.7. A vertex-weight function $w$ for a hypergraph $H$ assigns a weight $w(v) \geq 0$ to each vertex $v$ of $H$. We say that $w$ is a fractional vertex cover of $H$ if for each edge $e \in E(H), \Sigma_{v \in e} w(v) \geq 1$ holds. For a vertex-weight function $w$ for hypergraph $H$, we denote by weight $(w)$ its total weight, i.e., $\Sigma_{v \in V(H)} w(v)$. The fractional vertex cover number $\tau^{*}(H)$ is defined as the minimum weight $(w)$ where $w$ ranges over all fractional vertex covers of $H$. The vertex support $v$ supp $(w)$ of a hypergraph $H$ under a vertex-weight function $w$ is defined as $v \operatorname{supp}(w)=\{v \in V(H) \mid w(v)>0\}$.

For our result on bounded support, we will exploit the well-known dualities $\rho^{*}(H)=\tau^{*}\left(H^{d}\right)$ and $\tau^{*}(H)=\rho^{*}\left(H^{d}\right)$, where $H^{d}$ denotes the dual of $H$. To make optimal use of this, we make, for the moment, several assumptions. First, we will assume that (1) hypergraphs have no isolated vertices and (2) no empty edges. Furthermore, we assume that (3) hypergraphs never have two distinct vertices of the same "edge-type" (i.e., we exclude that two vertices occur in precisely the same edges) and (4) they never have two distinct edges of the same "vertex-type" (i.e., we exclude duplicate edges).

Assumptions (1)-(4) can be safely made. Recall that we are ultimately interested in the computation of an FHD of width $\leq k$ for given $k$. As mentioned above, without assumption (1), the computation of an edge-weight function and, hence, of an FHD of width $\leq k$ makes no sense. Assumption (2) does not restrict the search for a specific FHD, since we would never define an edge-weight function with non-zero weight on an empty edge. As far as assumption (3) is concerned, suppose that a hypergraph $H$ has groups of multiple vertices of identical edge-type. Then 
it is sufficient to consider the reduced hypergraph $H^{-}$resulting from $H$ by "fusing" each such group to a single vertex. Obviously $\rho^{*}(H)=\rho^{*}\left(H^{-}\right)$, and each edge-weight function for $H^{-}$can be extended in the obvious way to an edge-weight function of the same total weight to $H$. Finally, assumption (4) can also be made w.l.o.g., since we can again define a reduced hypergraph $\mathrm{H}^{-}$resulting from $H$ by retaining only one edge from each group of identical edges. Then every edge cover of $H^{-}$is an edge cover of $H$. Conversely, every edge cover of $H$ can be turned into an edge cover of $H^{-}$by assigning to each edge $e$ in $H^{-}$the sum of the weights of $e$ and all edges identical to $e$ in $H$.

Under our above assumptions (1)-(4), for every hypergraph $H$, the property $H^{d d}=H$ holds and there is an obvious one-to-one correspondence between the edges (vertices) of $H$ and the vertices (edges) of $H^{d}$. Moreover, there is an obvious one-to-one correspondence between the fractional edge covers of $H$ and the fractional vertex covers of $H^{d}$. In particular, if there is a fractional edge cover $\gamma$ for $H$, then its corresponding "dual" $\gamma^{d}$ assigns to each vertex $v$ of $H^{d}$ the same weight as to the edge in $H$ that is represented by this vertex and vice versa.

Note that if we do not make assumptions (3) and (4), then there are hypergraphs $H$ with $H^{d d} \neq H$. For instance, consider the hypergraph $H_{0}$ with $V\left(H_{0}\right)=\{a, b, c\}$ and $E\left(H_{0}\right)=\{e=\{a, b, c\}\}$, i.e., property (3) is violated. The hypergraph $H_{0}^{d}$ has a unique vertex $e$ and a unique hyperedge $\{e\}$. Hence, $H_{0}^{d d}$ is (isomorphic to) the hypergraph with a unique vertex $a$ and a unique hyperedge $\{a\}$, which is clearly different from the original hypergraph $H_{0}$.

To get an upper bound on the support $\operatorname{supp}(\gamma)$ of a fractional edge cover of a hypergraph $H$, we make use of the following result for fractional vertex covers. This result is due to Zoltán Füredi [26], who extended earlier results by Chung et al. [16]. Below, we appropriately reformulate Füredi's result for our purposes:

Proposition 6.8 ([26], PAge 152, Proposition 5.11.(III)). For every hypergraph $H$ of rank (i.e., maximal edge size) $r$, and every fractional vertex cover $w$ for $H$ satisfying weight $(w)=\tau^{*}(H)$, the property $|\operatorname{vsupp}(w)| \leq r \cdot \tau^{*}(H)$ holds.

By duality, exploiting the relationship $\rho^{*}(H)=\tau^{*}\left(H^{d}\right)$ and by recalling that the degree of $H$ corresponds to the rank of $H^{d}$, we immediately get the following corollary:

Corollary 6.9. For every hypergraph $H$ of degree $d$, and every fractional edge cover $\gamma$ for $H$ satisfying weight $(\gamma)=\rho^{*}(H)$, the property $|\operatorname{supp}(\gamma)| \leq d \cdot \rho^{*}(H)$ holds.

Proof of Theorem 6.6. Let $\mathscr{C}$ be a class of hypergraphs with degree at most $d$ and let $H \in \mathscr{C}$. Observe that we can extend Corollary 6.9 to any vertex subset $V^{\prime} \subseteq V(H)$ because $\rho^{*}\left(V^{\prime}\right)$ in $H$ is the same as $\rho^{*}\left(H\left[V^{\prime}\right]\right)$. The degree of the induced subhypergraph can not become greater than the degree of $H$.

Therefore, for every set of vertices $V^{\prime} \subseteq V(H)$, if $\rho^{*}\left(V^{\prime}\right) \leq k$, then $V^{\prime}$ can be fractionally covered using at most $k d$ edges. Thus, $f h w(H) \leq k$ if and only if there exists a width $k$ FHD of $H$ where the edge cover $\gamma_{u}$ of every node $u$ has $\left|\operatorname{supp}\left(\gamma_{u}\right)\right| \leq k d$. By Lemma 6.5, this can be decided in polynomial time.

\subsection{Computing FHDs under Bounded Intersection}

We will now follow the same strategy used in the previous section on bounded degree to prove tractability of checking fhw for hypergraph classes enjoying the BIP. However, bounded intersection width has different structural implications than bounded degree. Example 6.1 illustrated our main challenge in the fractional setting, namely, the potentially unbounded size of the support of an optimal fractional edge cover. In that example, the growth of the support is linked to the increase in the degree of $v_{0}$, whereas the intersection width remains 1 , no matter how large $n$ 
becomes. A combinatorial result that bounds the size of the support in terms of the optimal weight of the cover and the intersection width is therefore impossible.

Instead, we will show that if the optimal weight of a fractional cover $\gamma$ is below some fixed bound $k$, then we can find another fractional cover $v$ of weight $\leq k$ that can be expressed by a combination of edges and vertices, such that the number of both is bounded by functions of $k$ and the intersection width $i$. Analogously to the case of bounded degree, we will then derive tractability of the CHeск(FHD, $k$ ) problem via Lemma 6.5, which relies on the bounded support of $v$.

Our proof ultimately relies on two key observations:

(1) If all edges in a fractional cover $\gamma$ have weight bounded by some constant, then also the size of $B(\gamma)$ is bounded by some constant. In Lemma 6.10, we will make the relationship between the bound on the weights of individual edges and the bound on the size of $B(\gamma)$ precise.

(2) If an edge has weight close to 1 in an optimal cover, then we can in fact set this weight to 1 at the expense of a negligible increase of the weight. In Lemma 6.13, we will show under which circumstances we may increase the weight of an individual edge and still stay below $k$ with the total weight of $\gamma$.

We start by formalizing the first observation mentioned above.

Lemma 6.10. Let $k \geq 1, i \in \mathbb{N}$, and $\delta>0$ be constants, let $H$ be a hypergraph with iwidth $(H) \leq i$ and let $\gamma$ be an edge-weight function of $H$ with weight $(\gamma) \leq k$. Moreover, let $\gamma(e) \leq 1-\delta$ for every edge e. Then $|B(\gamma)| \leq \frac{i k^{2}}{\delta}$ holds.

Proof. For an arbitrary edge $e \in \operatorname{supp}(\gamma)$, let $m_{e}=|B(\gamma) \cap e|$. We first show that $m_{e} \leq \frac{i k}{\delta}$. Indeed, we are assuming that $\gamma(e) \leq 1-\delta$ holds. That is, $e$ puts weight $\leq 1-\delta$ on each vertex in $B(\gamma) \cap e$. Hence, weight $\geq \delta$ has to be put on each vertex in $B(\gamma) \cap e$ by the other edges. In total, the other edges thus have to put weight $\geq m_{e} \delta$ on the $m_{e}$ vertices in $B(\gamma) \cap e$.

By the upper bound $i$ on the intersection width, whenever $\gamma$ puts weight $w$ on some edge $e^{\prime}$ different from $e$, then, in total, at most weight $i w$ is put on the vertices in $e$. Since we are assuming weight $(\gamma) \leq k$, the total weight of all edges in $E(H)$ is $\leq k$. Hence, in total, at most weight $i k$ can be put on the vertices in $B(\gamma) \cap e$ by the edges different from $e$. We therefore have $m_{e} \delta \leq i k$ or, equivalently, $m_{e} \leq \frac{i k}{\delta}$.

Now let $m$ be the maximum size of $B(\gamma) \cap e$ attainable for any edge $e \in \operatorname{supp}(\gamma)$. Moreover, for an arbitrary edge $e \in \operatorname{supp}(\gamma)$, suppose that $\gamma(e)=w$ holds. Then, in total, $e$ puts weight $\leq m w$ on the vertices in $B(\gamma)$. Hence, the total weight put by all edges of $\operatorname{supp}(\gamma)$ on all vertices in $B(\gamma)$ is $\leq m k$. Moreover, every vertex in $B(\gamma)$ receives weight at least 1 . Hence, $|B(\gamma)| \leq m k$. Together with the above upper bound $\frac{i k}{\delta}$ on the size of $B(\gamma) \cap e$ for every edge $e$, we thus get $|B(\gamma)| \leq \frac{i k^{2}}{\delta}$.

Our next goal is to show that every fractional cover $\gamma$ can be replaced by a fractional cover that is, in a sense, very close to an integral cover. To formalize this closeness to an integral cover, we introduce the notion of $c$-bounded fractional part.

Definition 6.11. For a hypergraph $H$, edge weight function $\gamma: E(H) \rightarrow[0,1]$, and $E^{\prime} \subseteq \operatorname{supp}(\gamma)$, we write $\left.\gamma\right|_{E^{\prime}}$ to denote the restriction of $\gamma$ to $E^{\prime}$, i.e., $\left.\gamma\right|_{E^{\prime}}(e)=\gamma(e)$ if $e \in E^{\prime}$ and $\left.\gamma\right|_{E^{\prime}}(e)=0$ otherwise.

Now let $E^{\prime}=\left\{e \in \operatorname{supp}\left(\gamma_{u}\right) \mid \gamma_{u}(e)<1\right\}$ and let $c \in \mathbb{N}$. We say that $\gamma$ has $c$-bounded fractional part, if $\left|B\left(\left.\gamma_{u}\right|_{E^{\prime}}\right)\right| \leq c$.

In Lemma 6.13, we will formalize also the second observation from above. To this end, we first introduce the following terminology, which will be useful in the proof of Lemma 6.13: 
Definition 6.12. For $d \in \mathbb{N}$ and rational $k \geq 1$, we define:

$$
\begin{aligned}
\mathcal{H}(d) & =\{H=(V, E)|| V \mid \leq d\}, \\
M(k, d) & =\left\{\rho^{*}(H)-k \mid H \in \mathcal{H}(d) \text { and } \rho^{*}(H)>k\right\}, \\
\mu(k, d) & =\min (M(k, d)), \text { if } M(k, d) \neq \emptyset \text { and undefined otherwise. }
\end{aligned}
$$

Lemma 6.13. Let $k \geq 0$ be a rational number and $i \in \mathbb{N}$. Moreover, let $H$ be a hypergraph with $i$ width $(H) \leq i$ and let $S \subseteq V(H)$ with $S \neq \emptyset$. Then there exists a positive rational $\epsilon(k, i)$ and an integer $f(k, i)$ such that the following holds: If $\rho^{*}(S) \leq k+\epsilon(k, i)$, then $\rho^{*}(S) \leq k$ and there exists an edge weight function $v: E(H) \rightarrow[0,1]$ with the following properties:

- $S \subseteq B(v)$,

- weight $(v) \leq k$, and

- $v$ has c-bounded fractional part with $c=f(k, i)$.

Proof. The proof is by induction on $k$. For $0 \leq k<1$, we set $\epsilon(k, i)=\frac{1-k}{2}$ and $f(k, i)=0$. Then the statement vacuously holds, since we have $k+\epsilon(k, i)<1$ and there cannot be $S \neq \emptyset$ with $\rho^{*}(S)<1$. For $k \geq 1$, we define

$$
\begin{aligned}
\delta & =\frac{\epsilon(k-1, i) .}{2}, \\
d & =\frac{i k^{2}}{\delta}=\frac{2 i k^{2}}{\epsilon(k-1, i) .}, \\
f(k, i) & =\max (d, f(k-1, i)),
\end{aligned}
$$

and we choose $\epsilon(k, i)$ with $\epsilon(k, i)<\frac{\epsilon(k-1, i)}{2}$ and, if $\mu(k, d)$ is defined, also $\epsilon(k, i)<\mu(k, d)$.

Now consider $S \neq \emptyset$ with $\rho^{*}(S) \leq k+\epsilon(k, i)$ and let $\gamma$ be an optimal fractional cover of $S$, i.e., weight $(\gamma)=\rho^{*}(S)$ and $S \subseteq B(\gamma)$. We distinguish two cases:

Case 1. Suppose that there exists $e \in \operatorname{supp}(\gamma)$ with $\gamma(e)>1-\delta$. Consider $W=S \backslash e$. Then, we have

$$
\begin{aligned}
\rho^{*}(W) & \leq \rho^{*}(S)-\gamma(e) \leq k+\epsilon(k, i)-(1-\delta)=k+\epsilon(k, i)-1+\frac{\epsilon(k-1, i) .}{2} \leq \\
& \leq k+\frac{\epsilon(k-1, i) .}{2}-1+\frac{\epsilon(k-1, i) .}{2}=(k-1)+\epsilon(k-1, i) .
\end{aligned}
$$

Thus, by the induction hypothesis, there exists an edge weight function $v_{W}: E(H) \rightarrow[0,1]$ with the following properties:

- $W \subseteq B\left(v_{W}\right)$,

- $\operatorname{weight}\left(v_{W}\right) \leq k-1$, and

- $v_{W}$ has $c_{w}$-bounded fractional part with $c_{W}=f(k-1, i)$.

Then, we can extend $v_{W}$ to the desired edge cover $v$ of $S$ by setting $v(e)=1$ and $v\left(e^{\prime}\right)=v_{W}\left(e^{\prime}\right)$ for every edge $e^{\prime}$ with $e^{\prime} \neq e$.

Case 2. Suppose that $\gamma(e) \leq 1-\delta$ for every $e \in \operatorname{supp}(\gamma)$. Then, by Lemma $6.10,|B(\gamma)| \leq \frac{i k^{2}}{\delta}$. We claim that $\gamma$ is in fact the desired edge cover of $S$ with $c$-bounded fractional part for $c=$ $f(k, i)$. Indeed, by the above definitions of $\delta, d$, and $f(k, i)$, we have $|B(\gamma)| \leq \frac{i k^{2}}{\delta}$ and $f(k, i) \geq d$

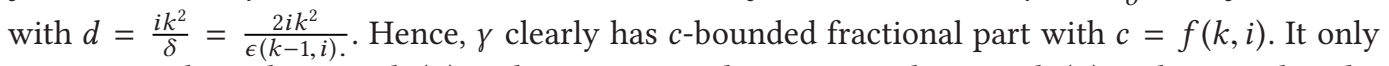
remains to show that weight $(\gamma) \leq k$. Suppose to the contrary that weight $(\gamma)>k$. Note that the subhypergraph of $H$ induced by $S$ is in $\mathcal{H}(d)$ with $d=\frac{i k^{2}}{\delta}=\frac{2 i k^{2}}{\epsilon(k-1, i) \text {. }}$ and $\gamma$ is an optimal fractional 
edge cover of $S$. Hence, $M(k, d)=\left\{\rho^{*}(H)-k \mid H \in \mathcal{H}(d)\right.$ and $\left.\rho^{*}(H)>k\right\}$ is non-empty and, therefore, $\mu(k, d)=\min (M(k, d))$ is defined. Moreover, $\rho^{*}(S)>k$ implies $\rho^{*}(S) \geq k+\mu(k, d)$. However, we have chosen $\epsilon(k, i)$ with $\epsilon(k, i)<\mu(k, d)$. Hence, from $\rho^{*}(S) \leq k+\epsilon(k, i)$ it follows that $\rho^{*}(S)<k+\mu(k, d)$ holds, a contradiction.

If an edge cover $\gamma$ of weight $\leq k$ has $c$-bounded fractional part, then $B(\gamma)$ is of the form $B(\gamma)=$ $e_{1} \cup \cdots \cup e_{\ell} \cup U$ with $\ell \leq k,|U| \leq c$, and $\{e \in E \mid \gamma(e)=1\}=\left\{e_{1}, \ldots, e_{\ell}\right\}$. W.l.o.g., we may assume $U \cap e_{j}=\emptyset$ for every $j$. Hence, there are at most $2^{c}$ "significantly different" edges in $\operatorname{supp}(\gamma)$ as far as covering $U$ is concerned, that is $|\{e \cap U \mid e \in \operatorname{supp}(\gamma)\}| \leq 2^{c}$. Hence, $\gamma$ can be replaced by an edge weight function $\gamma^{\prime}$ with weight $\left(\gamma^{\prime}\right)=$ weight $(\gamma), B(\gamma) \subseteq B\left(\gamma^{\prime}\right)$ and $\gamma^{\prime}$ has support bounded by $k+2^{c}$. We thus immediately obtain the following corollary, which may be of independent interest:

Corollary 6.14. Let $H$ be a hypergraph with iwidth $(H) \leq i$ and let $\gamma: E(H) \rightarrow[0,1]$ with weight $(\gamma) \leq k$. Then there exists an assignment $v: E(H) \rightarrow[0,1]$ such that weight $(v) \leq k, B(\gamma) \subseteq$ $B(v)$ and $v$ has bounded support (depending on $i$ and $k$ ).

Analogously to the case of bounded degree dealt with in Theorem 6.6, we now combine Corollary 6.14 with Lemma 6.5 to prove the main theorem of this subsection:

THEOREM 6.15. For every hypergraph class $\mathscr{C}$ that enjoys the BIP, and for every rational constant $k \geq 1$, the $\operatorname{CHEсK}(F H D, k)$ problem is tractable, i.e., given a hypergraph $H \in \mathscr{C}$, it is feasible in polynomial time to check $f h w(H) \leq k$ and, if so, to compute an FHD of width $k$ of $H$.

Proof. Let $\mathscr{C}$ be a class of hypergraphs with intersection width at most $i$ and let $H \in \mathscr{C}$. By Corollary 6.14, there exists a constant $q$, such that $f h w(H) \leq k$ holds if and only if there exists an FHD of width at most $k$ where for every node $u$, the cover $\gamma_{u}$ has $\left|\operatorname{supp}\left(\gamma_{u}\right)\right| \leq q$. Thus, we can again decide $f h w(H) \leq k$ via the bounded support case, which is tractable according to Lemma 6.5.

Deciding the Снеск Problem for Hypergraphs of Bounded Rank. We conclude this section by discussing an important special case of bounded intersection width: bounded rank. It is easy to see that for every hypergraph $H$, we have $i$ width $(H) \leq \operatorname{rank}(H)$. The tractability of the СНеск(FHD, $k$ ) problem for classes of bounded rank is therefore already a consequence of Theorem 6.15. However, the complexity of the algorithm described above may be prohibitive for practical use. We therefore choose to briefly present a significantly simpler method for hypergraph classes with bounded rank.

Definition 6.16. We say that a hypergraph $H$ has the $r$-bounded rank property $(r-B R P)$ if $\operatorname{rank}(H) \leq r$ holds. For a class $\mathscr{C}$ of hypergraphs, we say that $\mathscr{C}$ has the bounded rank property (BRP) if there exists a constant $r$ such that every hypergraph $H$ in $\mathscr{C}$ has the $r$-BRP.

For every hypergraph with $\operatorname{rank}(H) \leq r$ for some constant $r$, the following lemma is immediate:

LeмmA 6.17. Let $H$ be a hypergraph whose rank is bounded by some constant $r$. Then for every (fractional) edge weight function $\gamma$ for $H$ satisfying weight $(\gamma) \leq k$, the property $|B(\gamma)| \leq r \cdot k$ holds.

We can now use the above lemma to directly generate the candidate bags for the algorithm from Theorem 4.5. For any hypergraph $H$, we simply compute the set $\mathrm{S}$ of all sets with at most $r \cdot k$ vertices that can be fractionally covered with weight $k$. Clearly, if $\operatorname{ComCTD}(\mathrm{S}) \neq \emptyset$, then there exists an FHD of width $k$. However, if $f h w(H) \leq k$, then there exists a ComNF FHD $\left\langle T,\left(B_{u}\right)_{u \in T},\left(\gamma_{u}\right)_{u \in T}\right\rangle$ of width $k$. This is easy to see from the proof of Lemma 5.9. The construction of a ComNF GHD there translates directly to FHDs. Hence, $\left\langle T,\left(B_{u}\right)_{u \in T}\right\rangle$ is a ComNF TD where every bag is in S, i.e., $\operatorname{ComCTD}(S) \neq \emptyset$. We thus get the following special case of Theorem 6.15: 
Corollary 6.18. For every hypergraph class $\mathscr{C}$ that has bounded rank, and for every rational constant $k \geq 1$, the $C_{H E C K}(F H D, k)$ problem is tractable, i.e., given a hypergraph $H \in \mathscr{C}$, it is feasible in polynomial time to check $f h w(H) \leq k$ and, if so, to compute an FHD of width $k$ of $H$.

\section{APPROXIMATION OF $f h w$ UNDER CONSTRAINTS}

We now turn our attention to approximations of the $f h w$. It is known from Reference [44] that a tractable cubic approximation of the $f h w$ always exists, i.e.: for $k \geq 1$, there exists a polynomialtime algorithm that, given a hypergraph $H$ with $f h w(H) \leq k$, finds an FHD of $H$ of width $O\left(k^{3}\right)$. In this section, we search for conditions that guarantee a better approximation of the $f h w$.

The natural first candidate for restricting hypergraphs is the BMIP. For the Снеск(GHD, $k$ ) problem, this restriction guarantees tractability. Furthermore, the BIP and the BDP also lead to tractability of the $\operatorname{CHEck}(\mathrm{FHD}, k)$ problem for fixed $k \geq 1$. Both of these properties are generalized by the BMIP, making it the natural next step. For the fractional case, we will show that a significantly better polynomial-time approximation of the $f h w$ than in the general case is possible for hypergraphs enjoying the BMIP. It is noteworthy that we can reuse the strategy from the exact cases without modifications for this task. Finally, we show that classes with bounded Vapnik-Chervonenkis (VC) dimension-an even more general property than the BMIP-also allow for a polynomial-time approximation of the $f h w$, but with slightly weaker guarantees.

\subsection{Approximation of $f h w$ under BMIP}

We first inspect the case of the bounded multi-intersection property. We will show that the BMIP allows for an arbitrarily close approximation of the $f h w$ in polynomial time. Formally, the main result of this section is as follows:

Theorem 7.1. Let $\mathscr{C}$ be a hypergraph class that enjoys the BMIP and let $k, \epsilon$ be arbitrary rational constants with $k \geq 1$ and $\epsilon>0$. Then there exists a polynomial-time algorithm that, given a hypergraph $H \in \mathscr{C}$ with $f h w(H) \leq k$, finds an FHD of $H$ of width $\leq k(1+\epsilon)$.

Even though we are now interested in approximations, we can still employ the same strategy that was used in the previous section. However, it is not clear how to find a bounded representation of the sets that can be covered with weight $k$. Yet, if we introduce an arbitrarily small constant $\epsilon>0$, then it is possible to find supersets with weight $k(1+\epsilon)$ that are indeed representable in the desired fashion, as we will show in Lemma 7.3 below. The proof of this lemma will make use of the following combinatorial result:

Lemma 7.2. Fix an integer $c \geq 1$. Let $X=\left(x_{1}, \ldots, x_{n}\right)$ be a sequence of positive numbers $\leq \delta$ and fix $w$ such that $\sum_{j=1}^{n} x_{j} \geq w \geq \delta c$. Then, we have $\sum x_{i_{1}} \cdot x_{i_{2}} \cdots \cdots x_{i_{c}} \geq(w-\delta c)^{c}$, where the sum is over all c-tuples $\left(i_{1}, \ldots, i_{c}\right)$ of distinct integers from $[n]$.

Proof. We proceed by induction over $c$. For $c=1$ the statement holds by the requirement that $\sum_{j=1}^{n} x_{j} \geq w$. Suppose the statement holds for $c-1$. Let us consider only the tuples with a fixed value for $i_{c}$ and let us consider only the sum over those tuples, which we can write as $x_{i_{c}} \cdot \sum x_{i_{1}} \cdot x_{i_{2}} \cdots \cdots x_{i_{c-1}}$. Notably, the sum now ranges only over all $(c-1)$-tuples of distinct integers from $[n] \backslash\left\{i_{c}\right\}$. Therefore, we need to apply the induction hypothesis for the values in $X$, except for $x_{i_{c}}$. In particular, we apply the hypothesis with $w^{\prime}:=w-\delta$ instead of $w$ as the lower bound in the premise. The lower bound $w^{\prime}$ for the sum over the values in $X$, except for $x_{i_{c}}$, holds, since $x_{i_{c}} \leq \delta$ by assumption. Applying the induction hypothesis in this way then gives us the following inequality:

$$
x_{i_{c}} \cdot \sum x_{i_{1}} \cdot x_{i_{2}} \cdots x_{i_{c-1}} \geq x_{i_{c}}((w-\delta)-\delta(c-1))^{c-1}=x_{i_{c}}(w-\delta c)^{c-1} .
$$


Now, take the sum over all values in $[n]$ for $i_{c}$ to again consider all the $c$-tuples, and we arrive at the statement:

$$
\sum x_{i_{1}} \cdot x_{i_{2}} \cdots \cdots x_{i_{c}} \geq(w-\delta c)^{c-1} \sum_{j=1}^{n} x_{j} \geq w(w-\delta c)^{c-1} \geq(w-\delta c)^{c} .
$$

LEMmA 7.3. Let $H$ be a hypergraph with $c$-miwidth $(H) \leq i$ for some $c \geq 1$ and $i \geq 0$ such that $H$ is closed under intersection of edges and contains all edges of size 1. Moreover, fix $a k \geq 1$ and an $\epsilon$ with $0<\epsilon \leq 1$. Then there exists an integer $q$, depending only on $k, c, i$, and $\epsilon$, such that for any set of vertices $S$ with $\rho^{*}(S) \leq k$, there exists a q-set $S^{\prime}$ with $S \subseteq S^{\prime}$ and $\rho^{*}\left(S^{\prime}\right) \leq k(1+\epsilon)$.

Proof. Let $\gamma$ be a fractional edge cover of some $S \subseteq V(H)$ with weight $(\gamma) \leq k$. Similar to the proof for $\operatorname{CHEck}(\mathrm{FHD}, \mathrm{k})$ under BIP, we will partition the edges with non-zero weight into heavy and light edges. This time the threshold for heavy edges is chosen to be $\epsilon /(4 c)$, i.e., the sets $E_{\ell}$ and $E_{h}$ are defined as follows:

$$
\begin{aligned}
& E_{\ell}=\{e \in \operatorname{supp}(\gamma) \mid \gamma(e)<\epsilon /(4 c)\}, \\
& E_{h}=\{e \in \operatorname{supp}(\gamma) \mid \gamma(e) \geq \epsilon /(4 c)\} .
\end{aligned}
$$

Let $X$ be the set of all vertices for which the weight of $\left.\gamma\right|_{E_{h}}$ is at least $1-\epsilon / 2$ and let $S^{\prime}=S \cup X$. We will show that $S^{\prime}$ has the desired properties by showing that $X$ and $S \backslash X$ are $q_{1}$ and $q_{2}$ sets for appropriately chosen $q_{1}$ and $q_{2}$, both of which depend only on $k, c, i$, and $\epsilon$.

First, we can increase the weight of each edge in $\gamma$ by the factor $(1+\epsilon)$ to obtain a new $\gamma^{\prime}$ with $S^{\prime} \subseteq B\left(\gamma^{\prime}\right)$ and weight $\left(\gamma^{\prime}\right) \leq k(1+\epsilon)$. Now, in $\gamma^{\prime}, X$ is fully covered by the edges in $E_{h}$, i.e., $X \subseteq B\left(\left.\gamma^{\prime}\right|_{E_{h}}\right)$. This is due to the fact that $(1+\epsilon)(1-\epsilon / 2)=1+\epsilon / 2-\epsilon^{2} / 2 \geq 1$ holds by the assumption $0<\epsilon \leq 1$, i.e., if $\left.\gamma\right|_{E_{h}}$ put at least $1-\epsilon / 2$ weight on $X$, then $\left.\gamma^{\prime}\right|_{E_{h}}$ will put at least weight 1 on $X$. Since we are assuming weight $(\gamma) \leq k$ and $\gamma(e) \geq \epsilon /(4 c)$ for every $e \in E_{h}$, there can be no more than $4 c k / \epsilon$ edges in $E_{h}$. From Lemma 6.3, we then know that $X$ is a $\left(2^{4 c k / \epsilon}\right)$-set.

To complete the proof, we show that $S \backslash X$ contains at most $f(c, k, i, \epsilon)$ vertices and can therefore be represented as a union of that many edges of size 1 . By the definition of $X$, the vertices in $S \backslash X$ need to receive at least $\epsilon / 2$ weight from edges in $E_{\ell}$. As every light edge contributes $\leq \epsilon /(4 c)$ weight, there need to be at least $2 c$ light edges incident to every vertex in $S \backslash X$.

We proceed with a counting argument. Imagine a bipartite graph $G=(S \backslash X, T, E(G))$ where $T$ is the set of all $c$-tuples of distinct light edges. There is an edge from $v \in S \backslash X$ to $\left(e_{1}, \ldots, e_{c}\right) \in T$ iff $v$ is in $e_{1} \cap \cdots \cap e_{c}$. Furthermore, we assign weight $\prod_{j=1}^{c} \gamma\left(e_{j}\right)$ to every edge in $E(G)$ incident to a $\left(e_{1}, \ldots, e_{c}\right) \in T$.

We now count the total weight in $G$ from both sides. First observe that on the $T$ side, we have degree at most $i$ because $c$-miwidth $(H) \leq i$. Therefore, the total weight is at most $i$. $\sum_{\left(e_{1}, \ldots, e_{c}\right) \in T} \prod_{j=1}^{c} \gamma\left(e_{j}\right)$. Observe that $\sum_{\left(e_{1}, \ldots, e_{c}\right) \in T} \prod_{j=1}^{c} \gamma\left(e_{j}\right) \leq\left(\sum_{e_{1} \in E_{\ell}} \gamma\left(e_{1}\right)\right) \cdots \cdots\left(\sum_{e_{c} \in E_{\ell}} \gamma\left(e_{c}\right)\right)$ as, by distributivity, all the terms of the left-hand side sum are also present on the right-hand side of the inequality. Furthermore, we have $\sum_{e \in E_{\ell}} \gamma(e) \leq k$ and thus, by putting it all together, we see that the total weight in $G$ is at most $k^{c} i$.

From the $S \backslash X$ side, consider an arbitrary vertex $v \in S \backslash X$ and let $e_{1}, \ldots, e_{n}$ be the light edges in $E(H)$ containing $v$. As $v$ is not in $X$, the heavy edges of $\gamma$ contribute less than $1-\epsilon / 2$ weight to $v$. Hence, we have $\sum_{j=1}^{n} \gamma\left(e_{j}\right) \geq \epsilon / 2$ and $\gamma\left(e_{j}\right)<\epsilon /(4 c)$ for each $j \in[n]$. We can apply Lemma 7.2 for $X=\left\{\gamma\left(e_{1}\right), \ldots, \gamma\left(e_{n}\right)\right\}, \delta=\epsilon /(4 c)$, and $w=\epsilon / 2$ to get the inequality $\sum \gamma\left(e_{j_{1}}\right) \cdots \cdots \gamma\left(e_{j_{c}}\right) \geq$ $(\epsilon / 2-c \epsilon /(4 c))^{c}=(\epsilon / 4)^{c}$, where the sum ranges over all $c$-tuples $\left(e_{j_{1}}, \ldots, e_{j_{c}}\right)$ of distinct edges from $\left\{e_{1}, \ldots, e_{n}\right\}$, which denote the light edges in $E(H)$ containing $v$. 
We conclude that $v$ (now considered as a vertex in $G$ ) is incident to edges whose total weight is $\geq(\epsilon / 4)^{c}$ in $E(G)$. Since we have seen above that the total weight of all edges in $E(G)$ is $\leq k^{c} i$, there can be no more than $i(4 k / \epsilon)^{c}$ vertices in $S \backslash X$.

Proof of Theorem 7.1. We proceed analogously to the proofs of the two tractability results for $f h w$ in Section 6. Recall that, for every $H \in \mathscr{C}$, we are assuming $c$-miwidth $(H) \leq i$ for constants $c$ and $i$. Let $H$ be a hypergraph in $\mathscr{C}$ and let $H^{\prime}$ be the new hypergraph obtained by computing $H^{\bigcap}$ and adding every edge of size 1. As argued before, $f h w\left(H^{\prime}\right)=f h w(H)$ and, by Lemma 6.4, we can compute $H^{\prime}$ in polynomial time.

From the combination of Lemma 7.3 with Lemma 6.5, we have that there exists a polynomial time computable set $\mathrm{S}$ with $\operatorname{ComCTD}(\mathrm{S}) \neq \emptyset$ if and only if $f h w\left(H^{\prime}\right) \leq k(1+\epsilon)$. We can then use Theorem 4.5 to decide $\operatorname{ComCTD}(\mathrm{S}) \neq \emptyset$ and, in consequence, $f h w\left(H^{\prime}\right) \leq k(1+\epsilon)$ and, therefore, also $f h w(H) \leq k(1+\epsilon)$.

A polynomial time approximation scheme for finding optimal FHDs. Recall the definition of the $K$-Bounded-FHW-Optimization problem from Section 1, i.e.: Given a hypergraph $H$, we are interested in $f h w(H)$, but only if $f h w(H) \leq K$. We will now show that, with the algorithm from Theorem 7.1 at our disposal, we are able to give a polynomial time approximation scheme for the bounded optimization problem. More precisely, we aim at an approximation algorithm with the following properties:

Definition 7.4 (PTAS $[9,53])$. Let $\Pi$ be an (intractable) minimization problem with positive objective function $f_{\Pi}$. An algorithm Alg is called an approximation scheme for $\Pi$ if on input $(I, \epsilon)$, where $I$ is an instance of $\Pi$ and $\epsilon>0$ is an error parameter, it outputs a solution $s$ such that:

$$
f_{\Pi}(I, s) \leq(1+\epsilon) \cdot f_{\Pi}\left(I, s^{*}\right),
$$

where $s^{*}$ is an optimal solution of $I$, i.e., for all other solutions $s^{\prime}$ of $I$ it holds that $f_{\Pi}\left(I, s^{*}\right) \leq$ $f_{\Pi}\left(I, s^{\prime}\right)$.

Alg is called a polynomial time approximation scheme (PTAS) if for every fixed $\epsilon>0$, its running time is bounded by a polynomial in the size of instance $I$.

We now show that, in case of the BMIP, the K-BOUNDED-FHW-OptimizATION problem indeed allows for a PTAS:

THEOREM 7.5. For every hypergraph class $\mathscr{C}$ that enjoys the BMIP, there exists a PTAS for the K-BOUNDED-FHW-OPTIMIZATION problem.

Proof. By Theorem 7.1, there exists a function find-fhd $(H, k, \epsilon, c, i)$ with the following properties:

- find-fhd takes as input a hypergraph $H$ with $c$-miwidth $(H) \leq i$ and numbers $k \geq 1, \epsilon \geq 0$;

- find-fhd returns an FHD $\mathcal{F}$ of $H$ of width $\leq k+\epsilon$ if $f h w(H) \leq k$ or fail. In the latter case it is guaranteed that $f h w(H)>k$. Note that finding an FHD of width $\leq k+\epsilon$ is equivalent to finding an FHD of width $\leq k\left(1+\epsilon^{\prime}\right)$ for $\epsilon^{\prime}=\epsilon / k$.

- find-fhd runs in time polynomial in the size of $H$, where $k, \epsilon, c$, and $i$ are considered as fixed.

We can then obtain the required PTAS by calling find-fhd $\left(H, k^{\prime}, \epsilon / 2, c, i\right)$ for every width $k^{\prime}=$ $a \cdot \frac{\epsilon}{2}+1$ such that $1 \leq k^{\prime} \leq K+\epsilon / 2$ and $a$ is a non-negative integer. The procedure returns the lowest $k^{\prime}$ (and the respective FHD) for which find-fhd accepts. Since this requires no more than $2 K / \epsilon$ calls to find-fhd, the procedure is clearly polynomial.

It is also not difficult to verify the correctness of the procedure. If $f h w(H) \leq K$, then find-fhd is called for a width $k^{\prime}$, such that $f h w(H) \leq k^{\prime} \leq f h w(H)+\epsilon / 2$. By Theorem 7.1, this call returns 
an FHD $\mathcal{F}$ width at most $k^{\prime}+\epsilon / 2 \leq f h w(H)+\epsilon$. However, suppose our procedure returns width $k^{\prime}$ and the FHD $\mathcal{F}$ with width at most $k^{\prime}+\epsilon / 2$. By definition, $k^{\prime}$ is the lowest width for which find-fhd accepted, hence find-fhd rejected for the next lowest parameter $k^{\prime}-\epsilon / 2$. Therefore, we can deduce that $k^{\prime}-\epsilon / 2 \leq f h w(H)$ and thus also $k^{\prime} \leq f h w(H)+\epsilon / 2$.

\subsection{Approximation of $f h w$ under Bounded VC Dimension}

We now present a polynomial-time approximation of the $f h w$ for classes of hypergraphs enjoying bounded Vapnik-Chervonenkis (VC) dimension. This bounded VC dimension is yet again more general property than BMIP but the approximation guarantees in this class will be slightly weaker. We will combine some classical results on the VC dimension with some novel observations. This will yield an approximation of the $f h w$ up to a logarithmic factor. We first recall the definition of the VC-dimension of hypergraphs.

Definition 7.6 ([49,52]). Let $H=(V(H), E(H))$ be a hypergraph and $X \subseteq V(H)$ a set of vertices. Denote by $\left.E(H)\right|_{X}$ the set $\left.E(H)\right|_{X}=\{X \cap e \mid e \in E(H)\}$. The vertex set $X$ is called shattered if $\left.E(H)\right|_{X}=2^{X}$. The $V C$ dimension $\operatorname{vc}(H)$ of $H$ is the maximum cardinality of a shattered subset of $V(H)$.

We now provide a link between the VC-dimension and our approximation of the fhw.

Definition 7.7. Let $H=(V(H), E(H))$ be a hypergraph. A transversal (also known as hitting set) of $H$ is a subset $S \subseteq V(H)$ that has a non-empty intersection with every edge of $H$. The transversality $\tau(H)$ of $H$ is the minimum cardinality of all transversals of $H$.

Clearly, $\tau(H)$ corresponds to the minimum of the following integer linear program: Find a mapping $w: V \rightarrow\{0,1\}$ that minimizes $\Sigma_{v \in V(H)} w(v)$ under the condition that $\Sigma_{v \in e} w(v) \geq 1$ holds for each hyperedge $e \in E$.

The fractional transversality $\tau^{*}$ of $H$ is defined as the minimum of the above linear program when dropping the integrality condition, thus allowing mappings $w: V \rightarrow \mathbb{R}_{\geq 0}$. Finally, the transversal integrality gap tigap $(H)$ of $H$ is the ratio $\tau(H) / \tau^{*}(H)$.

Recall that computing the mapping $\lambda_{u}$ for some node $u$ in a GHD can be seen as searching for a minimal edge cover $\rho$ of the vertex set $B_{u}$, whereas computing $\gamma_{u}$ in an FHD corresponds to the search for a minimal fractional edge cover $\rho^{*}$ [35]. Again, these problems can be cast as linear programs where the first problem has the integrality condition and the second one has not. Further, we can define the cover integrality gap cigap $(H)$ of $H$ as the ratio $\rho(H) / \rho^{*}(H)$. With this, we state the following approximation result for $f h w$ :

THEOREM 7.8. Let $\mathscr{C}$ be a class of hypergraphs with VC-dimension bounded by some constant d and let $k \geq 1$. Then there exists a polynomial-time algorithm that, given a hypergraph $H \in \mathscr{C}$ with fhw $(H) \leq k$, finds an FHD of $H$ of width $O(k \cdot \log k)$.

Proof. The proof proceeds in several steps.

Reduced hypergraphs. Recall the notion of reduced hypergraphs from Section 6.2. There, it was established that we can assume, w.l.o.g., that every hypergraph is reduced. For the rest of this proof, we therefore consider only reduced hypergraphs. This ensures that $\left(H^{d}\right)^{d}=H$ holds. It is well-known and easy to verify that the following relationships between $H$ and $H^{d}$ hold for any reduced hypergraph $H$, (see, e.g., Reference [21]):

(1) The edge coverings of $H$ and the transversals of $H^{d}$ coincide.

(2) The fractional edge coverings of $H$ and the fractional transversals of $H^{d}$ coincide.

(3) $\rho(H)=\tau\left(H^{d}\right), \rho^{*}(H)=\tau^{*}\left(H^{d}\right)$, and cigap $(H)=\operatorname{tigap}\left(H^{d}\right)$. 
VC-dimension. By a classical result (Reference [20], Theorem (5.4); see also Reference [12] for related results), for every hypergraph $H=(V(H), E(H))$ with at least two edges, we have:

$$
\operatorname{tigap}(H)=\tau(H) / \tau^{*}(H) \leq 2 \mathrm{vc}(H) \log \left(11 \tau^{*}(H)\right)
$$

For hypergraphs $H$ with a single edge only, $\mathrm{vc}(H)=0$, and thus the above inequation does not hold. However, for such hypergraphs $\tau(H)=\tau^{*}(H)=1$. By putting this together, we get:

$$
\operatorname{tigap}(H)=\tau(H) / \tau^{*}(H) \leq \max \left(1,2 \mathrm{vc}(H) \log \left(11 \tau^{*}(H)\right)\right)
$$

Moreover, in Reference [7], it is shown that $\mathrm{vc}\left(H^{d}\right)<2^{\mathrm{vc}(H)+1}$ always holds. In total, we thus get

$$
\begin{aligned}
\operatorname{cigap}(H)=\operatorname{tigap}\left(H^{d}\right) & \leq \max \left(1,2 \mathrm{vc}\left(H^{d}\right) \log \left(11 \tau^{*}\left(H^{d}\right)\right)\right) \\
& \leq \max \left(1,2^{\mathrm{vc}(H)+2} \log \left(11 \rho^{*}(H)\right)\right) \\
& \leq \max \left(1,2^{d+2} \log \left(11 \rho^{*}(H)\right)\right), \text { which is } O\left(\log \rho^{*}(H)\right) .
\end{aligned}
$$

Approximation of fhw by $g h w$. Suppose that $H$ has an FHD $\left\langle T,\left(B_{u}\right)_{u \in V(T)},(\lambda)_{u \in V(T)}\right\rangle$ of width $k$. Then there exists a GHD of $H$ of width $O(k \cdot \log k)$. Indeed, we can find such a GHD by leaving the tree structure $T$ and the bags $B_{u}$ for every node $u$ in $T$ unchanged and replacing each fractional edge cover $\gamma_{u}$ of $B_{u}$ by an optimal integral edge cover $\lambda_{u}$ of $B_{u}$. By the above inequality, we thus increase the weight at each node $u$ only by a factor $O(\log k)$. Moreover, we know from Reference [4] that $h w(H) \leq 3 \cdot g h w(H)+1$ holds. In other words, we can compute an HD of $H$ (which is a special case of an FHD) in polynomial time, whose width is $O(k \cdot \log k)$.

To conclude, the following Lemma 7.9 establishes a relationship between BMIP and VCdimension:

Lemma 7.9. If a class $\mathscr{C}$ of hypergraphs has the BMIP, then it has bounded VC-dimension. However, there exist classes $\mathscr{C}$ of hypergraphs with bounded VC-dimension that do not have the BMIP.

Proof. [BMIP $\Rightarrow$ bounded VC-dimension.] Let $c \geq 1, i \geq 0$ and let $H$ be a hypergraph with $c$-miwidth $(H) \leq i$. We claim that then $\mathrm{vc}(H) \leq c+i$ holds.

Assume to the contrary that there exists a set $X \subseteq V$, such that $X$ is shattered and $|X|>c+i$. We pick $c$ arbitrary, pairwise distinct vertices $v_{1}, \ldots, v_{c}$ from $X$ and define $X_{j}=X \backslash\left\{v_{j}\right\}$ for each $j$. Then $X=\left(X_{1} \cap \cdots \cap X_{c}\right) \cup\left\{v_{1}, \ldots, v_{c}\right\}$ holds and also $|X| \leq\left|X^{*}\right|+c$ with $X^{*} \subseteq X_{1} \cap \cdots \cap X_{c}$.

Since $X$ is shattered, for each $1 \leq j \leq c$, there exists a distinct edge $e_{j} \in E(H)$ with $X_{j}=X \cap e_{j}$. Hence, $X_{j}=X \backslash\left\{v_{j}\right\} \subseteq e_{j}$ and also $X^{*} \subseteq e_{1} \cap e_{2} \cap \cdots \cap e_{c}$ holds, i.e., $X^{*}$ is in the intersection of $c$ edges of $H$. By $c$-miwidth $(H) \leq i$, we thus get $\left|X^{*}\right| \leq i$. In total, we have $|X| \leq\left|X^{*}\right|+c \leq i+c$, which contradicts our assumption that $|X|>c+i$ holds.

[bounded VC-dimension $\nRightarrow$ BMIP.] It suffices to exhibit a family $\left(H_{n}\right)_{n \in \mathbb{N}}$ of hypergraphs such that $\mathrm{vc}\left(H_{n}\right)$ is bounded, whereas $c$-miwidth $\left(H_{n}\right)$ is unbounded for any constant $c$. We define $H_{n}=$ $\left(V_{n}, E_{n}\right)$ as follows:

$$
\begin{aligned}
& V_{n}=\left\{v_{1}, \ldots, v_{n}\right\}, \\
& E_{n}=\left\{V_{n} \backslash\left\{v_{i}\right\} \mid 1 \leq i \leq n\right\} .
\end{aligned}
$$

Clearly, vc $\left(H_{n}\right)<2$. Indeed, take an arbitrary set $X \subseteq V$ with $|X| \geq 2$. Then $\emptyset \subseteq X$ but $\emptyset \neq X \cap e$ for any $e \in E_{n}$. However, let $c \geq 1$ be an arbitrary constant and let $X=e_{i_{1}} \cap \cdots \cap e_{i_{\ell}}$ for some $\ell \leq c$ and edges $e_{i_{j}} \in E_{n}$. Obviously, $|X| \geq n-c$ holds. Hence, also $c$-miwidth $\left(H_{n}\right) \geq n-c$, i.e., it is not bounded by any constant $i \geq 0$.

In the first part of Lemma 7.9, we have shown that $\mathrm{vc}(H) \leq c+i$ holds. For an approximation of an FHD by a GHD, we need to approximate the fractional edge cover $\gamma_{u}$ of each bag $B_{u}$ by an integral edge cover $\lambda_{u}$, i.e., we consider fractional vs. integral edge covers of the induced hypergraphs 
$H_{u}=\left(B_{u}, E_{u}\right)$ with $E_{u}=\left\{e \cap B_{u} \mid e \in E(H)\right\}$. Obviously, the bound $\mathrm{vc}(H) \leq c+i$ carries over to $\operatorname{vc}\left(H_{u}\right) \leq c+i$.

\section{CONCLUSION AND FUTURE WORK}

In this work, we have settled the complexity of deciding $f h w(H) \leq k$ for fixed constant $k \geq 2$ and $g h w(H) \leq k$ for $k=2$ by proving the NP-completeness of both problems. This gives negative answers to two open problems. On the positive side, we have identified rather mild restrictions such as the BDP (i.e., the bounded degree property), BIP (i.e., the bounded intersection property) LogBIP, BMIP (i.e., the bounded multi-intersection property), and LogBMIP, which give rise to a PTIME algorithm for the CHeck(GHD, $k$ ) problem. Moreover, we have shown that the BDP and the BIP ensure tractability also of the $\operatorname{CHEck}(\mathrm{FHD}, k)$ problem. For the BMIP, we have shown that an arbitrarily close approximation of the $f h w$ in polynomial time exists. In case of bounded VC dimension, we have proposed a polynomial-time algorithm for approximating the $f h w$ up to a logarithmic factor. As the empirical analyses reported in Reference [23] show, these restrictions are very well-suited for instances of CSPs and, even more so, of CQs. We believe that they deserve further attention.

Our work does not finish here. We plan to explore several further issues regarding the computation and approximation of the fractional hypertree width. We find the following questions particularly appealing: (i) Does the special condition defined by Grohe and Marx [35] lead to tractable recognizability also for FHDs, i.e., in case we define "sc- $f h w(H)$ " as the smallest width an FHD of $H$ satisfying the special condition, can $s c-f h w(H) \leq k$ be recognized efficiently? (ii) Our tractability result in Section 5 for the CHEck(FHD, $k$ ) problem is weaker than for CHECK(GHD, $k$ ). In particular, for the BMIP, we have only obtained efficient approximations of the $f h w$. Note that the techniques applied in the tractability proof of $\mathrm{CHECK}(\mathrm{FHD}, k)$ in case of the BIP do not carry over in an obvious way to the BMIP. An important property in our tractability proof is that edges with low weight can only contribute a constant number of vertices to $B(\gamma)$. This property crucially depends on the $\mathrm{BIP}$, which implies that the additional weight put by other edges on these vertices is bounded by $k i$. Between submission and publication of this manuscript, we were indeed able to generalize the tractability result for $\mathrm{CHECK}(\mathrm{FHD}, k)$ to hold also under the BMIP. We refer to the respective publication for further details [30].

\section{APPENDIX}

\section{A A POLYNOMIAL TIME ALGORITHM FOR THE COMNF CTD PROBLEM}

In this section, we provide a proof of Theorem 4.5. To do so, we present a bottom-up construction of ComNF CTDs, if they exist, using bottom-up dynamic programming. Note that our presentation does not optimize for runtime; our goal is only to establish that the problem can be decided in polynomial time. As before, for a hypergraph $H$ and $U \subseteq V(H)$, we write $H[U]$ for the subhypergraph induced by the vertices $U$.

Definition A.1. A pair $(B, C)$ of disjoint subsets of $V(H)$ is a block if $C$ is a [B]-component of $H$ or $C=\emptyset$. Such a block is headed by $B$. Let $(B, C)$ and $(X, Y)$ be two blocks. We say that $(X, Y) \leq(B, C)$ if $X \cup Y \subseteq B \cup C$ and $Y \subseteq C$.

In a bottom-up search for a decomposition, we can consider a block to correspond to a subproblem. Namely, a block $(B, C)$ corresponds to the subproblem of whether there exists a ComNF decomposition of $H[B \cup C]$, where the root has bag $B$. If such a decomposition exists, then we call a block satisfied. Note that if $C=\emptyset$, the block is trivially satisfied. Our overall goal will be to find a set $B$, such that all blocks headed by $B$ are satisfied. In the context of finding candidate TDs, we will eventually consider only those blocks that are headed by a candidate bag. 
Lemma A.2. Let $H$ be a hypergraph and $B \subseteq V(H)$. If all blocks headed by $B$ are satisfied, then $H$ has a ComNF tree decomposition where $B$ is the bag of the root.

Proof. Let $\left(B, C_{1}\right), \ldots,\left(B, C_{\ell}\right)$ be all the blocks headed by $B$. Since each of the blocks is satisfied, there exist ComNF TDs $\left\langle T_{1},\left(B_{1, u}\right)_{u \in T_{1}}\right\rangle, \ldots,\left\langle T_{\ell},\left(B_{\ell, u}\right)_{u \in T_{\ell}}\right\rangle$, respectively. Furthermore, the root of each such TD has $B$ as its bag.

The construction of a TD of $H$ is then straightforward by simply merging the individual trees at their common root node with bag $B$. It is staightforward to verify that this is indeed a valid TD. Any edge $e \in E(H)$ is a subset of $B \cup C_{i}$ for some $i$, since each block corresponds to a separate $[B]$-component. Thus, $e$ is already covered in the corresponding subtree. Furthermore, for distinct $i, j \in[\ell]$, we have that $C_{i}$ and $C_{j}$ are disjoint. Hence, any vertices that are shared by subtrees for two blocks are in $B$ and the connectedness therefore follows from the connectedness of each subtree.

In standard dynamic programming fashion, our algorithm will decide whether a block $(B, C)$ is satisfied by checking whether certain subproblems are satisfied. Intuitively, these subproblems are the blocks that correspond to the subtrees under the root of the decomposition for $H[B \cup C]$. However, because we will proceed bottom-up, some additional technical considerations need to be made to guarantee the tree decomposition properties. We capture this in the following notion of a basis of a block:

Definition A.3. For a block $(B, C)$ and vertex set $B^{\prime} \subseteq V(H)$ with $B^{\prime} \neq B$ and let $\left(B^{\prime}, C_{1}^{\prime}\right), \ldots,\left(B^{\prime}, C_{\ell}^{\prime}\right)$ be all the blocks headed by $B^{\prime}$ such that $\left(B^{\prime}, C_{i}^{\prime}\right) \leq(B, C)$ for all $i \in[\ell]$. We say that $B^{\prime}$ is a basis of $(B, C)$ if the following conditions hold:

(1) $C \subseteq B^{\prime} \cup \bigcup_{i=1}^{\ell} C_{i}^{\prime}$,

(2) For each $e \in E(H)$ such that $e \cap C \neq \emptyset, e \subseteq B^{\prime} \cup \bigcup_{i=1}^{\ell} C_{i}^{\prime}$,

(3) For each $i \in[\ell]$, the block $\left(B^{\prime}, C_{i}^{\prime}\right)$ is satisfied.

The following two lemmas now show that this notion of basis indeed strongly corresponds to the existence of a tree decomposition. First, we show that if a block has a basis, then it is satisfied, demonstrating that the check can indeed be reduced to satisfiedness of subproblems. The second lemma shows that in any ComNF TD, child nodes form a basis of the block corresponding to their subtree. After these two lemmas, we are ready to state the full algorithm.

Lemma A.4. Let $(B, C)$ be a block and $B^{\prime}$ a basis of $(B, C)$. Then $(B, C)$ is satisfied.

Proof. Let $\left(B^{\prime}, C_{i}^{\prime}\right)$ for some $i>1$ be as in Definition A.3. For the sake of a simpler presentation, we will write $C^{\prime}$ for the set $B^{\prime} \cup \cup_{i=1}^{\ell} C_{i}^{\prime}$. First, observe that by the assumption in the statement and by Lemma A.2 there exists a ComNF TD $\mathcal{T}=\left\langle T,\left(B_{u}\right)_{u \in T}\right\rangle$ of $H\left[C^{\prime}\right]$ where $B^{\prime}$ is the bag of the root node.

Consider the TD $\mathcal{T}^{\prime}$ obtained by adding a new root node $u_{0}$ with bag $B$ to $\mathcal{T}$ and attaching the old root as the only child of $u_{0}$. We claim that $\mathcal{T}^{\prime}$ is a ComNF TD of $H[B \cup C]$, and thus that $(B, C)$ is satisfied.

To verify this claim, we first consider connectedness in $\mathcal{T}^{\prime}$. We know that the connectedness condition holds in $\mathcal{T}$. By construction, the only way the condition can fail in $\mathcal{T}^{\prime}$ is if there is a $v \in \bigcup_{i=1}^{\ell} C_{i}^{\prime}$ that is also in $B$. Suppose $v$ is in a $C_{i}^{\prime}$, then it is also in $C$ because $\left(B^{\prime}, C_{i}^{\prime}\right) \leq(B, C)$. But $C$ and $B$ are disjoint, hence, $v \notin B$ and $\mathcal{T}^{\prime}$ satisfies the connectedness condition.

We move on to showing that every edge of $H[B \cup C]$ is covered in $\mathcal{T}^{\prime}$. By the second condition of a basis, any $e \in E(H)$ is either fully covered by $C^{\prime}$, or disjoint with $C$. If we consider an edge $e^{\prime}=e \cap(B \cup C)$ in $H[B \cup C]$, then we can distinguish two cases. If $e^{\prime} \cap C \neq \emptyset$, then $e^{\prime} \subseteq C^{\prime}$ and 
thus $e^{\prime}$ (and actually even $e$ itself) is covered already in $\mathcal{T}$. Otherwise, if $e^{\prime} \cap C=\emptyset$, then $e^{\prime}=e \cap B$. Thus, $e^{\prime}$ is covered in $B$.

Finally, since all the blocks $\left(B^{\prime}, C_{i}^{\prime}\right)$ are $\leq(B, C)$, we have $C^{\prime} \subseteq B \cup C$. In particular, because we assume $C \subseteq C^{\prime}$, we also have $B \cup C^{\prime}=B \cup C$. Thus, $V\left(\mathcal{T}^{\prime}\right)=B \cup C$ and the claim holds.

Lemma A.5. Let $H$ be a hypergraph and $\left\langle T,\left(B_{u}\right)_{u \in T}\right\rangle$ be a ComNF TD of H. Let $r \in T$ be a nonleaf node. For each child $s$ of $r$, let $C_{s}$ be the $\left[B_{r}\right]$-component associated with $s$. The following two statements are true:

- $\left(B_{s}, D\right) \leq\left(B_{r}, C_{s}\right)$ if and only if $D$ is either a component associated with a child of s, or if $D=\emptyset$,

- $B_{s}$ is a basis of the block $\left(B_{r}, C_{s}\right)$.

Proof. We first observe that $\left(B_{s}, D\right) \leq\left(B_{r}, C_{s}\right)$ if and only if $D$ is either empty or a component associated with a child of $s$. Indeed, since we assume ComNF, we have that $V\left(T_{s}\right)=C_{s} \cup\left(B_{r} \cap B_{s}\right)$ and $C_{s}$ is the only such $\left[B_{r}\right]$-component. Thus, $V\left(T_{s}\right) \subseteq B_{r} \cup C_{s}$.

Moreover, $B_{s} \cup D \subseteq V\left(T_{s}\right)$ holds for every block $\left(B_{s}, D\right)$ where $D$ is empty or a component associated with a child of $s$, which completes the proof of the "if" direction. For the "only if" direction, recall that $\left(B_{s}, D\right) \leq\left(B_{r}, C_{s}\right)$ requires $D \subseteq C_{s}$. Since there is only one node associated with $C_{s}$, we conclude $D \subseteq V\left(T_{s}\right)$. Since $D$ is a $\left[B_{s}\right]$-component, it must have its own associated child of $s$.

Now that we know exactly which blocks headed by $B_{s}$ are relevant, we can show that they satisfy the conditions of a basis. Let child(s) be the set of all children of $s$. For every $u \in \operatorname{child}(s)$, let $D_{u}$ be the $\left[B_{s}\right]$-component associated with $u$. The vertices that occur in the subtree $T_{s}$ are precisely $V\left(T_{s}\right)=B_{s} \cup \bigcup_{u \in \operatorname{child}(s)} D_{u}$. Since we assume ComNF, we also have $C_{s} \subseteq V\left(T_{s}\right)$ and therefore Condition 1 of a basis is satisfied. Furthermore, it is straightforward to verify that for every $u \in \operatorname{child}(s)$, the subtree rooted at node $s$ with only $T_{u}$ attached below it, is a ComNF TD of $H\left[B_{s} \cup D_{u}\right]$. Hence, the block $\left(B_{s}, D_{u}\right)$ is satsified for every such $u$ and Condition 3 holds.

For Condition 2 it is enough to observe that if $e \cap C_{s} \neq \emptyset$, then it must be covered in the subtree $T_{s}$, i.e., $e \subseteq V\left(T_{s}\right)$. Otherwise, suppose $e$ were only covered in some node $u$ not in the subtree $T_{s}$. There is a vertex $v \in e \cap C_{s}$ that occurs in $V\left(T_{s}\right)$ but not in $B_{r}$ (recall $B_{r}$ and $C_{s}$ are disjoint). Any path from a node of $T_{s}$ to $u$ must pass through $B_{r}$, which would break connectedness for $v$.

Algorithm 2 describes the resulting procedure. We simply build, bottom-up, a list of satisfied blocks. The check whether " $B$ ' is a basis of $(B, C)$ " is to be understood as relative to the program state. That is, Condition 3 of a basis in Definition A.3 is checked w.r.t. whether the blocks are marked as satisfied in the algorithm.

Proof of Theorem 4.5. We only present the decision procedure. Soundness of the procedure follows immediately by Lemmas A.4 and A.2. Furthermore, it follows from the soundness argument that constructing an appropriate TD from an accepting state is trivial. Completeness of the procedure follows by Lemma A.5, which demonstrates that any $\mathcal{T} \in \operatorname{ComCTD}(\mathrm{S})$ can be found in this bottom-up fashion.

What is left to show is that Algorithm 2 indeed always terminated in polynomial time. Observe that a straightforward representation of $\mathrm{S}$ as a list of lists of vertices has size $O(|\mathrm{~S}| \cdot|V(H)|$. $\log |V(H)|)$. For asserting polynomial runtime it is therefore not necessary to distinguish between the size of the representation of $\mathbf{S}$ and $|\mathrm{S}|$. The set blocks has at most $|\mathrm{S}| \cdot|V(H)|$ elements and computing components is polynomial in the size of the representation of $H$. Furthermore, when we consider Condition 3 to refer to blocks that are marked as satisfied, deciding whether a set $B^{\prime}$ is a basis is also polynomial. Thus, the whole algorithm requires only polynomial time. 


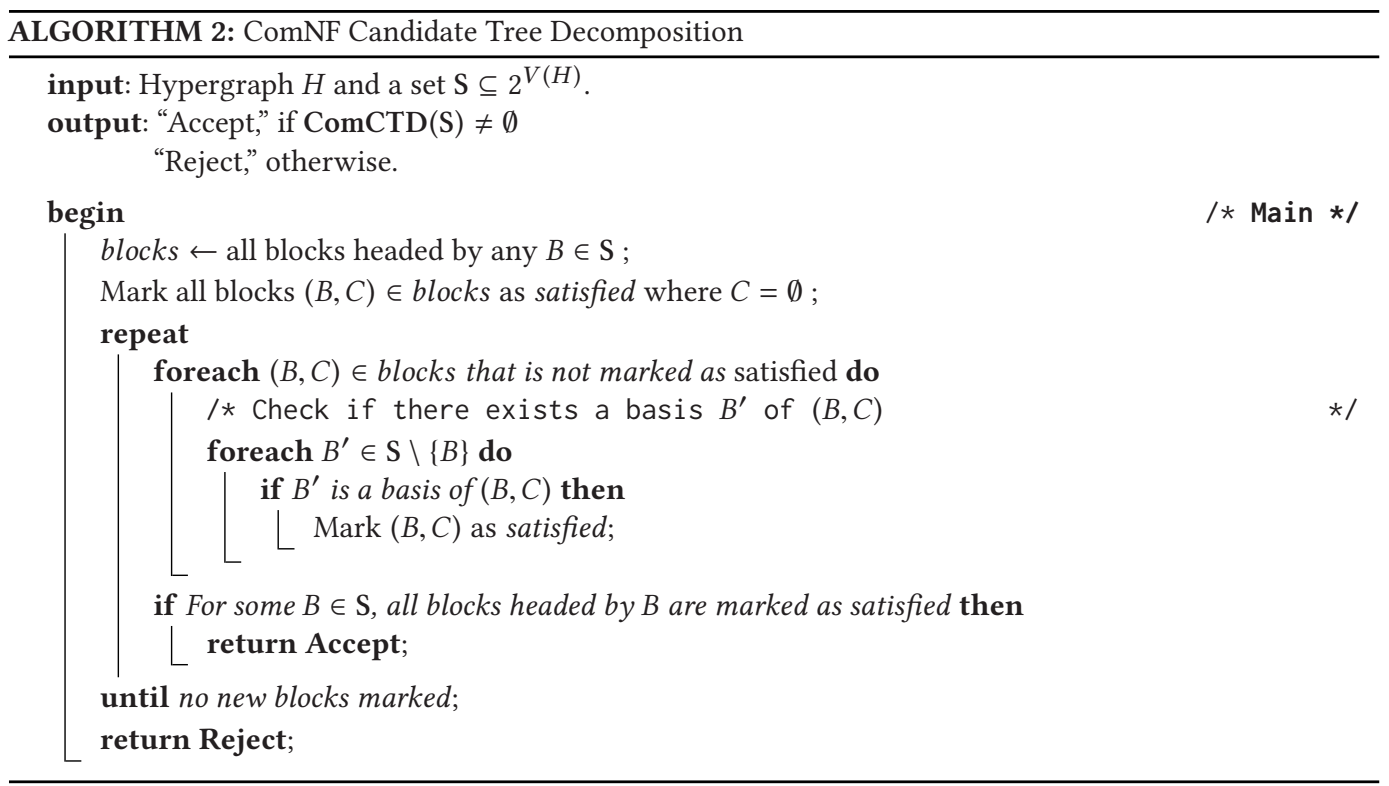

A LogCFL upper-bound. For the sake of simplicity, the algorithm presented here uses dynamic programming to establish a PTIME upper-bound. However, it is not difficult to see that the ComNF CTD problem lies in the class LogCFL and is therefore highly parallelizable: Consider the LogCFL algorithm for computing hypertree decompositions presented in Reference [31]. To guess the next separator, we now, roughly speaking, guess some element of S instead of guessing a set of up to $k$ edges. Since $S$ is an input, it is sufficient to guess an index into $S$. The argument for LogCFL membership of computing hypertree decompositions then also applies to computing ComNF CTDs.

The LogCFL upper-bound also extends to our main tractability result for GHDs. Observe that the set $\mathrm{S}$ in Lemma 5.18 can be computed in logarithmic space (for fixed $a, c$, and $k$ ). Hence, the proof of Theorem 5.4 also establishes LogCFL membership of computing GHDs of fixed width assuming the LogBMIP. For FHDs it remains open whether this applies. There, our approach requires the computation of the fractional cover number of sets of vertices (cf. Lemma 6.5). No LogCFL algorithm is known for this task.

\section{ACKNOWLEDGMENTS}

We are truly grateful to one of the anonymous referees, whose insightful and exceptionally deep review comments have greatly helped to simplify the presentation and increase readability.

\section{REFERENCES}

[1] Christopher R. Aberger, Susan Tu, Kunle Olukotun, and Christopher Ré. 2016. EmptyHeaded: A relational engine for graph processing. In Proceedings of SIGMOD. ACM, 431-446.

[2] Christopher R. Aberger, Susan Tu, Kunle Olukotun, and Christopher Ré. 2016. Old techniques for new join algorithms: A case study in RDF processing. CoRR abs/1602.03557 (2016).

[3] Isolde Adler. 2004. Marshals, monotone marshals, and hypertree-width. f. Graph Theor. 47, 4 (2004), 275-296.

[4] Isolde Adler, Georg Gottlob, and Martin Grohe. 2007. Hypertree width and related hypergraph invariants. Eur. f. Comb. 28, 8 (2007), 2167-2181.

[5] Foto N. Afrati, Manas Joglekar, Christopher Ré, Semih Salihoglu, and Jeffrey D. Ullman. 2017. GYM: A multiround join algorithm in MapReduce. In Proceedings of ICDT, Vol. 68. Schloss Dagstuhl - Leibniz-Zentrum fuer Informatik, $4: 1-4: 18$. 
[6] Molham Aref, Balder ten Cate, Todd J. Green, Benny Kimelfeld, Dan Olteanu, Emir Pasalic, Todd L. Veldhuizen, and Geoffrey Washburn. 2015. Design and implementation of the LogicBlox system. In Proceedings of SIGMOD. ACM 1371-1382.

[7] Patrick Assouad. 1983. Densité et dimension. Annales de l'Institut Fourier 33, 3 (1983), 233-282.

[8] Albert Atserias, Martin Grohe, and Dániel Marx. 2013. Size bounds and query plans for relational joins. SIAM 7. Comput. 42, 4 (2013), 1737-1767.

[9] Giorgio Ausiello. 1999. Complexity and Approximation: Combinatorial Optimization Problems and Their Approximability Properties. Springer. Retrieved from http://www.worldcat.org/oclc/249492438.

[10] Nurzhan Bakibayev, Tomás Kociský, Dan Olteanu, and Jakub Závodný. 2013. Aggregation and ordering in factorised databases. PVLDB 6, 14 (2013), 1990-2001.

[11] Angela Bonifati, Wim Martens, and Thomas Timm. 2017. An analytical study of large SPARQL query logs. PVLDB 11, 2 (2017), 149-161. Retrieved from http://www.vldb.org/pvldb/vol11/p149-bonifati.pdf.

[12] H. Brönnimann and M. T. Goodrich. 1995. Almost optimal set covers in finite VC-dimension. Discr. Comput. Geom. 14, 4 (01 Dec. 1995), 463-479. DOI : https://doi.org/10.1007/BF02570718

[13] Ashok K. Chandra and Philip M. Merlin. 1977. Optimal implementation of conjunctive queries in relational data bases. In Proceedings of STOC. ACM, 77-90.

[14] Chandra Chekuri and Anand Rajaraman. 2000. Conjunctive query containment revisited. Theor. Comput. Sci. 239, 2 (2000), 211-229.

[15] Hubie Chen and Víctor Dalmau. 2005. Beyond hypertree width: Decomposition methods without decompositions. In Proceedings of CP (Lecture Notes in Computer Science), Vol. 3709. Springer, 167-181.

[16] Fan R. K. Chung, Zoltan Fueredi, M. R. Garey, and Ronald L. Graham. 1988. On the fractional covering number of hypergraphs. SIAM f. Discr. Math. 1, 1 (1988), 45-49.

[17] David A. Cohen, Peter Jeavons, and Marc Gyssens. 2008. A unified theory of structural tractability for constraint satisfaction problems. F. Comput. Syst. Sci. 74, 5 (2008), 721-743. DOI : https://doi.org/10.1016/j.jcss.2007.08.001

[18] Víctor Dalmau, Phokion G. Kolaitis, and Moshe Y. Vardi. 2002. Constraint satisfaction, bounded treewidth, and finitevariable logics. In Proceedings of CP (Lecture Notes in Computer Science), Vol. 2470. Springer, 310-326.

[19] Rina Dechter and Judea Pearl. 1989. Tree clustering for constraint networks. Artif. Intell. 38, 3 (1989), 353-366.

[20] Guo-Li Ding, Paul Seymour, and Peter Winkler. 1994. Bounding the vertex cover number of a hypergraph. Combinatorica 14, 1 (1994), 23-34.

[21] Pierre Duchet. 1996. Hypergraphs. In Handbook of Combinatorics (Vol. 1). The MIT Press, 381-432.

[22] Ronald Fagin. 1983. Degrees of acyclicity for hypergraphs and relational database schemes. F. ACM 30, 3 (1983), 514550 .

[23] Wolfgang Fischl, Georg Gottlob, Davide M. Longo, and Reinhard Pichler. 2019. HyperBench: A benchmark and tool for hypergraphs and empirical findings. In Proceedings of PODS. ACM, 464-480.

[24] Wolfgang Fischl, Georg Gottlob, and Reinhard Pichler. 2018. General and fractional hypertree decompositions: Hard and easy cases. In Proceedings of PODS. ACM, 17-32. DOI:https://doi.org/10.1145/3196959.3196962.

[25] Eugene C. Freuder. 1990. Complexity of k-tree structured constraint satisfaction problems. In Proceedings of AAAI. AAAI Press/The MIT Press, 4-9.

[26] Zoltán Füredi. 1988. Matchings and covers in hypergraphs. Graphs Combinat. 4, 1 (1988), 115-206.

[27] Nathan Goodman and Oded Shmueli. 1984. The tree projection theorem and relational query processing. F. Comput. Syst. Sci. 28, 1 (1984), 60-79. DOI : https://doi.org/10.1016/0022-0000(84)90076-X

[28] Georg Gottlob and Gianluigi Greco. 2013. Decomposing combinatorial auctions and set packing problems. F. ACM 60, 4 (2013), 24.

[29] Georg Gottlob, Gianluigi Greco, and Francesco Scarcello. 2018. Tree projections and constraint optimization problems: Fixed-parameter tractability and parallel algorithms. J. Comput. Syst. Sci. 94 (2018), 11-40. DOI : https://doi.org/10.1016/ j.jcss.2017.11.005

[30] Georg Gottlob, Matthias Lanzinger, Reinhard Pichler, and Igor Razgon. 2020. Fractional covers of hypergraphs with bounded multi-intersection. In Proceedings of MFCS (Lecture Notes in Computer Science), Vol. 170. Schloss Dagstuhl Leibniz-Zentrum für Informatik, 41:1-41:14.

[31] Georg Gottlob, Nicola Leone, and Francesco Scarcello. 2002. Hypertree decompositions and tractable queries. F. Comput. Syst. Sci. 64, 3 (2002), 579-627. DOI : https://doi.org/10.1006/jcss.2001.1809

[32] Georg Gottlob, Zoltán Miklós, and Thomas Schwentick. 2009. Generalized hypertree decompositions: NP-hardness and tractable variants. F. ACM 56, 6 (2009), 30:1-30:32. DOI : https://doi.org/10.1145/1568318.1568320

[33] Martin Grohe. 2007. The complexity of homomorphism and constraint satisfaction problems seen from the other side. f. ACM 54, 1 (2007), 1:1-1:24. DOI : https://doi.org/10.1145/1206035.1206036

[34] Martin Grohe and Dániel Marx. 2006. Constraint solving via fractional edge covers. In Proceedings of SODA. ACM Press, 289-298. 
[35] Martin Grohe and Dániel Marx. 2014. Constraint solving via fractional edge covers. ACM Trans. Algor 11, 1 (2014), 4:1-4:20.

[36] Martin Grohe, Thomas Schwentick, and Luc Segoufin. 2001. When is the evaluation of conjunctive queries tractable? In Proceedings of STOC. ACM, 657-666.

[37] Marc Gyssens, Peter Jeavons, and David A. Cohen. 1994. Decomposing constraint satisfaction problems using database techniques. Artif. Intell. 66, 1 (1994), 57-89.

[38] Marc Gyssens and Jan Paredaens. 1984. A decomposition methodology for cyclic databases. In Advances in Data Base Theory: Volume 2. Springer, 85-122.

[39] Khayyam Hashmi, Zaki Malik, Erfan Najmi, and Abdelmounaam Rezgui. 2016. SNRNeg: A social network enabled negotiation service. Inf. Sci. 349 (2016), 248-262.

[40] Mahmoud Abo Khamis, Hung Q. Ngo, Christopher Ré, and Atri Rudra. 2015. Joins via geometric resolutions: Worstcase and beyond. In Proceedings of PODS. ACM, 213-228.

[41] Mahmoud Abo Khamis, Hung Q. Ngo, and Atri Rudra. 2016. FAQ: Questions asked frequently. In Proceedings of PODS. ACM, 13-28.

[42] Phokion G. Kolaitis and Moshe Y. Vardi. 2000. Conjunctive-query containment and constraint satisfaction. f. Comput. Syst. Sci. 61, 2 (2000), 302-332.

[43] Aviv Lustig and Oded Shmueli. 1999. Acyclic hypergraph projections. F. Algor. 30, 2 (1999), 400-422. DOI : https://doi. org/10.1006/jagm.1998.0965

[44] Dániel Marx. 2010. Approximating fractional hypertree width. ACM Trans. Algor. 6, 2 (2010), 29:1-29:17. DOI : https: //doi.org/10.1145/1721837.1721845

[45] Dániel Marx. 2011. Tractable structures for constraint satisfaction with truth tables. Theor. Comput. Syst. 48, 3 (2011), 444-464.

[46] Dániel Marx. 2013. Tractable hypergraph properties for constraint satisfaction and conjunctive queries. F. ACM 60, 6 (2013), 42:1-42:51.

[47] Lukas Moll, Siamak Tazari, and Marc Thurley. 2012. Computing hypergraph width measures exactly. Inf. Process. Lett. 112, 6 (2012), 238-242. DOI : https://doi.org/10.1016/j.ipl.2011.12.002

[48] Dan Olteanu and Jakub Závodný. 2015. Size bounds for factorised representations of query results. ACM Trans. Datab. Syst. 40, 1 (2015), 2:1-2:44. DOI : https://doi.org/10.1145/2656335

[49] Norbert Sauer. 1972. On the density of families of sets. F. Combinat. Theor. (A) 13, 1 (1972), 145-147.

[50] Susan Tu and Christopher Ré. 2015. DunceCap: Query plans using generalized hypertree decompositions. In Proceedings of SIGMOD. ACM, 2077-2078.

[51] René van Bevern, Rodney G. Downey, Michael R. Fellows, Serge Gaspers, and Frances A. Rosamond. 2015. MyhillNerode methods for hypergraphs. Algorithmica 73, 4 (2015), 696-729. DOI : https://doi.org/10.1007/s00453-015-9977-x

[52] Vladimir Vapnik and Alexey Chervonenkis. 1971. On the uniform convergence of relative frequencies of events to their probabilities. Theor. Probab. Appl. 16 (1971), 264-280.

[53] Vijay V. Vazirani. 2001. Approximation Algorithms. Springer. Retrieved from http://www.springer.com/computer/ theoretical+computer+science/book/978-3-540-65367-7.

[54] Mihalis Yannakakis. 1981. Algorithms for acyclic database schemes. In Proceedings of VLDB. IEEE Computer Society, $82-94$.

Received November 2018; revised January 2021; accepted March 2021

Journal of the ACM, Vol. 68, No. 5, Article 38. Publication date: September 2021. 